\title{
Global-Local Finite Element Modeling in Skewed Multi-girder Bridges
}

\author{
A Thesis \\ Presented to \\ The faculty of the School of Engineering and Applied Science \\ University of Virginia \\ In Partial Fulfillment \\ of the requirements for the Degree \\ Master of Science (Civil Engineering) \\ by \\ Heather B. Jones, E.I.T
}

May 2012 


\section{APPROVAL SHEET}

This thesis is submitted in partial fulfillment of the

Requirements for the degree of

Master of Science (Civil Engineering)

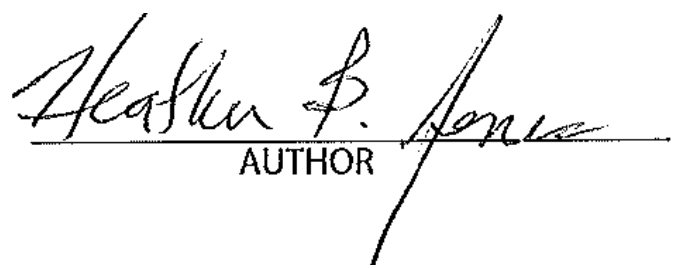

This thesis has been read and approved by the Examining Committee:

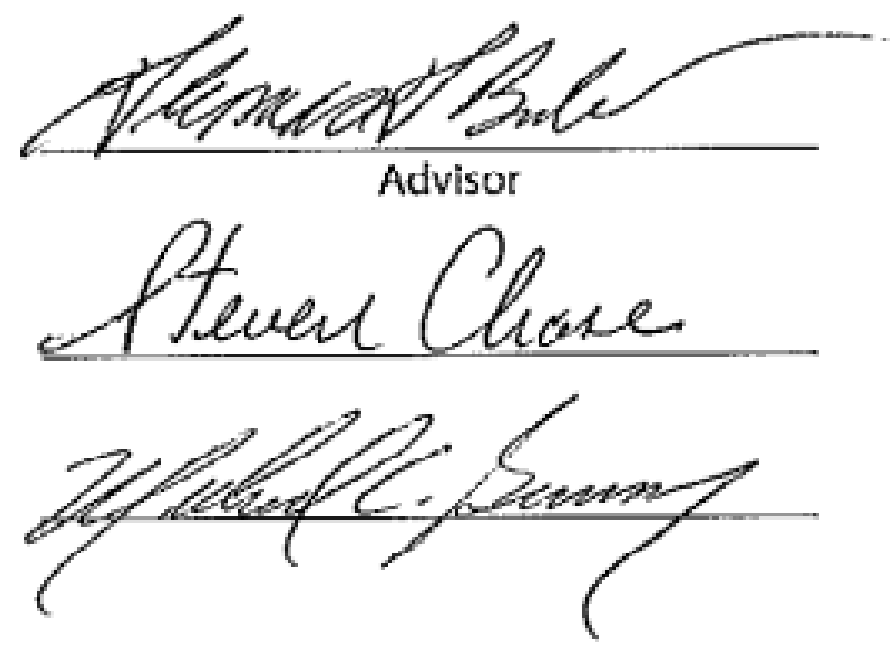

Accepted for the School of Engineering and Applied Science:

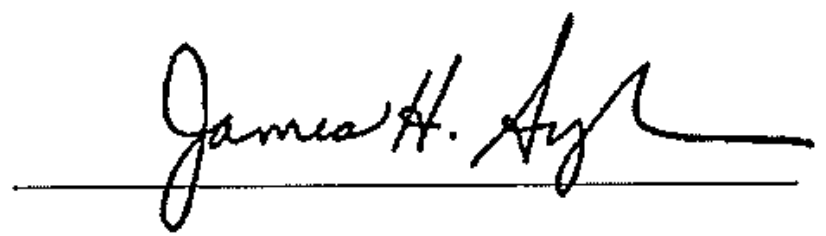

Dean, School of Engineering and Applied Science

May 


\section{Acknowledgements}

There are so many people in my life that have gotten me to where I am today. I would like to take this time to say thank you to them. First, I would like to thank my family. They have been so supportive with my school career. It has been difficult living so far from them, but they are wonderful at encouraging me to follow my dream and believing in me. I would like to not only thank my mom, Donna, my dad, Tom, and my brother, Travis, but my grandparents, Harris and Libby, as well for giving me the support and motivation to do my best. My other support system is my friends. Without Kristy, Amanda, and Miranda, I would not have made it through the two years. They are awesome people and wonderful friends. They kept me sane when I was stressed and always made me laugh. I could not ask for a better family and friends.

I would not be writing this thesis without my great education from the University of New Hampshire and the University of Virginia. I would like to personally thank Dr. Ricardo Medina for all his assistance and encouragement during my medical issues in my undergraduate degree. I would like to thank Dr. Ricardo Medina, Dr. Jean Benoit, and Dean Robert Henry for being great role models and wonderful teachers. At the University of Virginia, I would like to thank Dr. Tom Baber and Dr. Marek-Jerzy Pindera. Not only were they great instructors, but they took the time to see how their lectures could relate to my personal future career goals. I had the great honor of being Dr. Tom Baber's teaching and research assistant. I would like to thank him for those opportunities. I would also like to thank him for being my advisor. Without his knowledge and guidance, I would not have been able to finish this study on time. 


\section{Abstract}

Steel plate girder and multi-girder bridges are commonly used in the United States. There are approximately 9000 such bridges in Virginia alone. Of these, approximately 2600 are skewed bridges, which have their cross-members connecting adjacent girders at the piers and abutments.

Fatigue of welded steel bridges has become a significant problem in the United States in recent years. A number of different fatigue mechanisms have been identified. In particular, many steel plate girder bridges have been found to be vulnerable to out of plane web distortion, which can cause fatigue cracking where cross-frame members or diaphragms are connected to girders. The connection plates at these joints have been designed with a web gap at the top and bottom of the plate and it is common, but not universal, practice to not weld the connection plate to the tension flange. This gap can lead to web distortion that causes fatigue cracks. Out of plane web distortion fatigue cracking was observed in a web along a skewed cross-frame located at pier-10 in the Carter-Glass Bridge.

This thesis investigates the use of global-local finite element models to evaluate the fatigue potential of cross-frame to girder connections, with particular reference to the Carter-Glass Bridge, which has experienced a significant fracture at a skewed crossframe connection. A global model of this bridge was created previously. The objective of this thesis is to develop a local model that can identify the magnitude of stress in a common welded cross-frame to girder connection and to apply that model to the CarterGlass Bridge. The local model was developed to be versatile enough to act as a template 
for various skew angles and girder dimensions. The exterior skew connection of the Carter-Glass Bridge was the main finite element local model studied. This skewed connection occurs in a region of negative bending. The boundary conditions for a globallocal model and a partial-local model were analyzed. The ultimate goal was to determine the stress intensity factor for this complex three-dimensional geometry. 


\section{Table of Contents}

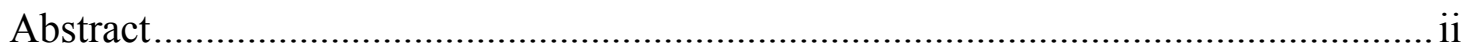

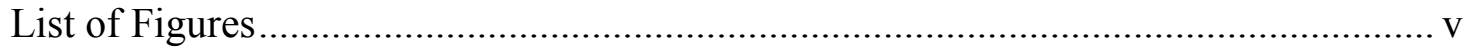

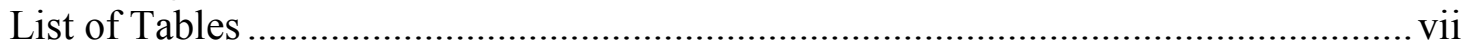

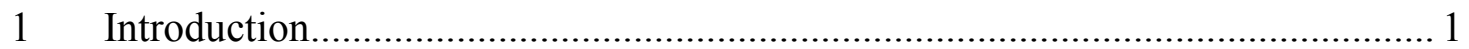

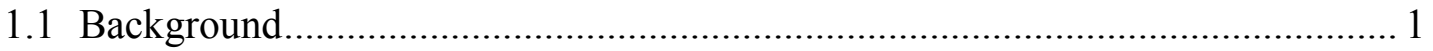

1.2 Web Distortion and Distortion Induced Cracking ............................................... 3

1.3 Fatigue Analysis and Fracture Mechanics......................................................... 4

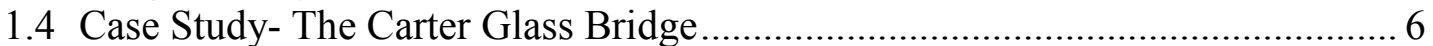

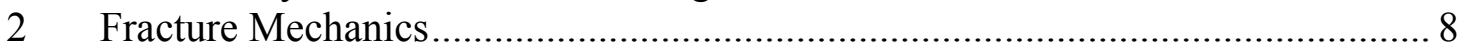

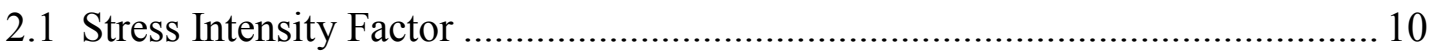

3 The Global-Local Modeling Approach …………............................................ 15

3.1 Global Modeling Options ............................................................................ 16

3.2 Partial Local versus Complete Local Modeling ………................................... 19

3.3 Global- Local Approach for the Carter Glass Bridge....................................... 21

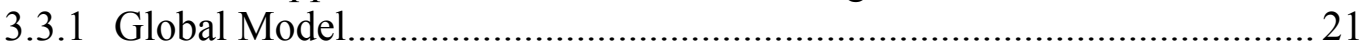

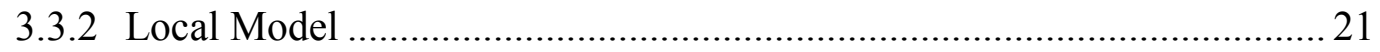

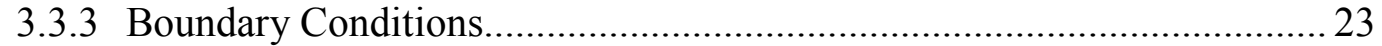

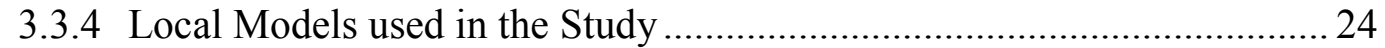

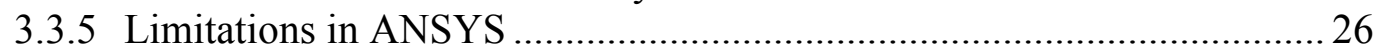

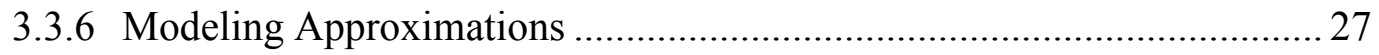

3.4 Preliminary Simplified Models ...................................................................... 29

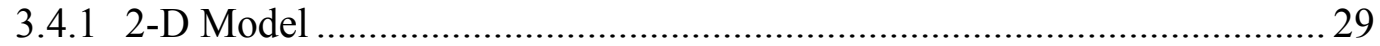

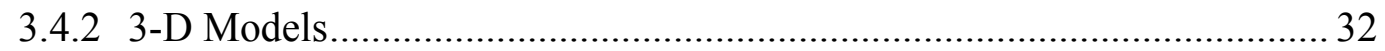

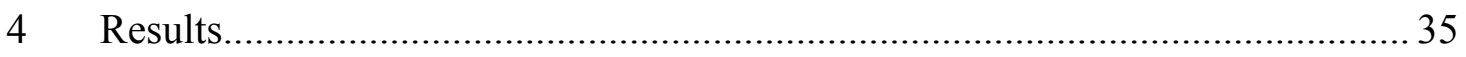

4.1 Preliminary Model Studies ....................................................................... 35

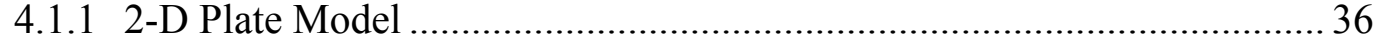

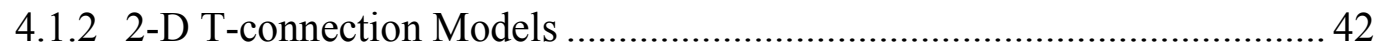

4.1.3 3-D T-connection Hot Spots ………………............................................ 57

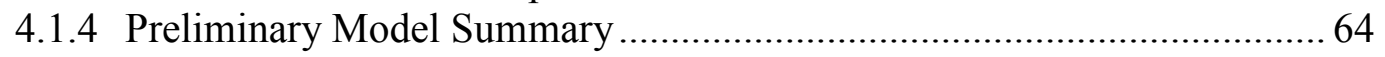

4.2 Results from Complete Local Model ..................................................................6 64

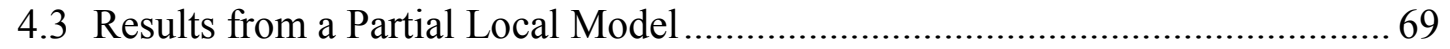

4.4 Web-Distortion Corrections .......................................................................... 73

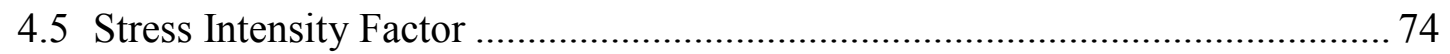

$5 \quad$ Summary and Conclusions .......................................................................... 78

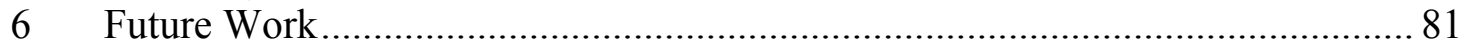

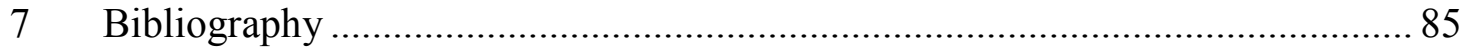

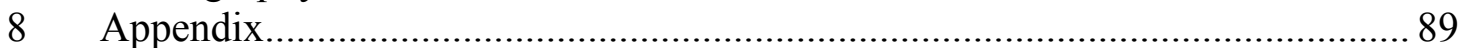

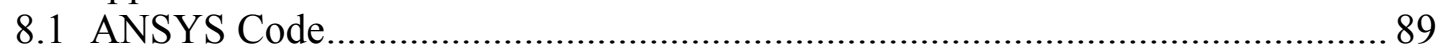

8.2 MATLAB Code for Interpolation .............................................................. 124

8.3 The Carter Glass Bridge Plans and Photos ..................................................... 144 


\section{List of Figures}

Figure 1: Multi-Girder Bridge with Cross-frames .......................................................... 1

Figure 2: Detail 1-Web distortion at the web gap between the flange and the connection plate weld in a negative bending region....................................................................... 3

Figure 3: Sections at Skewed Cross-Frames .................................................................. 4

Figure 4: Basic Modes of Crack Surface Displacements (Barsom and Rolfe, 1999) ......... 5

Figure 5: The Carter Glass Bridge, Lynchburg, VA ………………….......................... 6

Figure 6: Web Cracking in Plate Girder..................................................................... 7

Figure 7: Imperfections and Cracks in Fillet Weld (Barsom and Rolfe, 1999) ................. 9

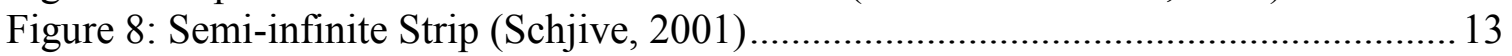

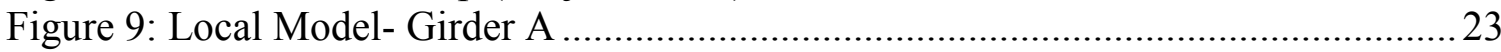

Figure 10: Stress Concentration Regions in Fillet Welds (Barsom and Rolfe, 1999) ...... 25

Figure 11: Plate Model (a) full model (b) section modeled .............................................. 30

Figure 12: 2-D T-connection Boundary Conditions ……………................................... 31

Figure 13: 3-D T-connection Dimensions for 8 Node Element, 20 Node Element, and

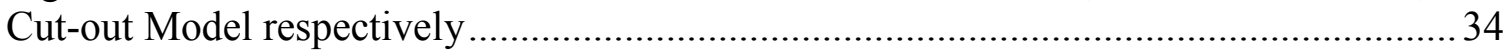

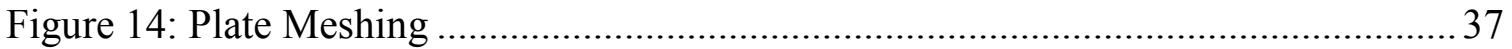

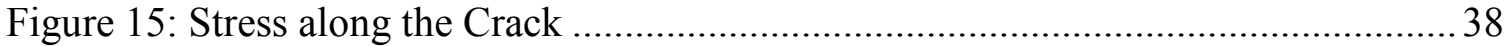

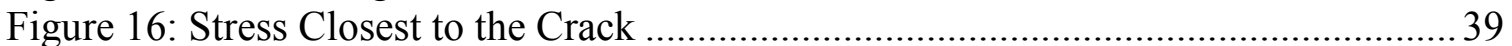

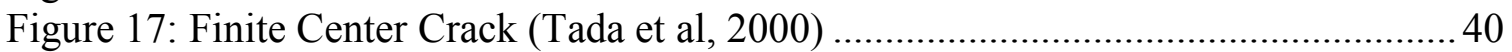

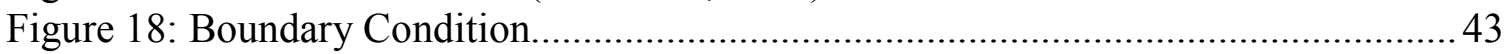

Figure 19: Meshing for (a) 4 Node Element by Key Points, (b) 4 Node Element by Nodes, and (c) 8 Node Element by Nodes .................................................................... 44

Figure 20: 4 Node Element Results By Key Points ....................................................... 45

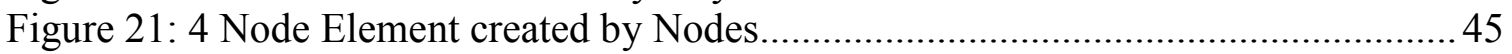

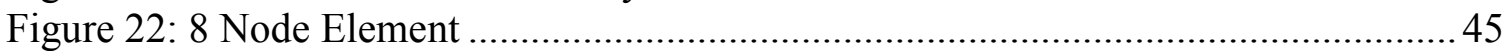

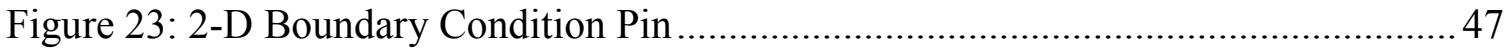

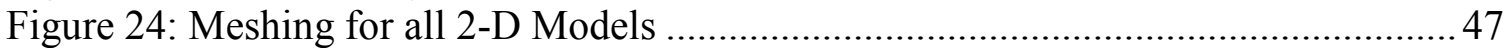

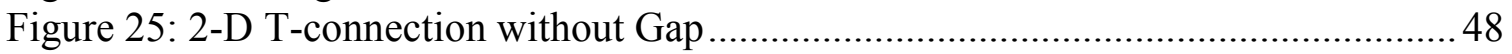

Figure 26: Fixed Boundary Conditions of 2-D T-connection ............................................4 49

Figure 27: Mesh for Four Boundary Conditions .......................................................... 51

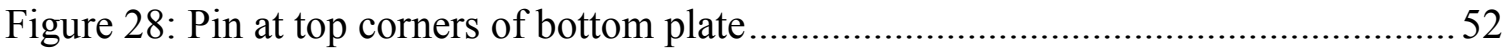

Figure 29: Pin at top corners of bottom plate and rollers along verticals .........................53

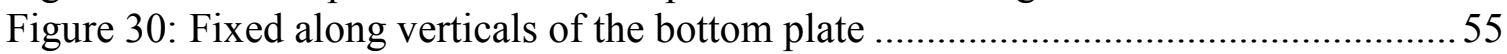

Figure 31: Fixed along bottom plate verticals and along bottom.....................................56

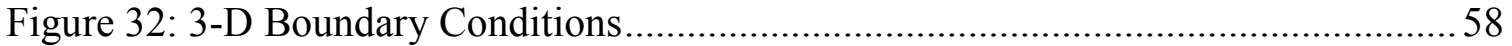

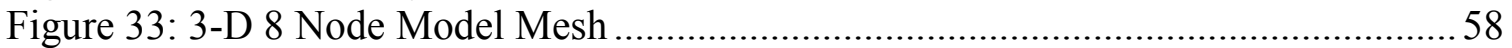

Figure 34: 3-D 8 Node Model Deflected Shape with Stress Contours ............................. 59

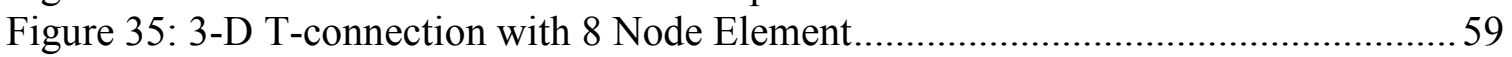

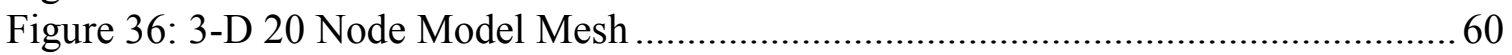

Figure 37: 20 Node Model Deflected Shape with Contours ................................................ 61

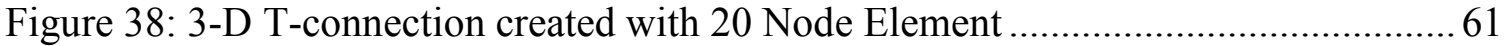

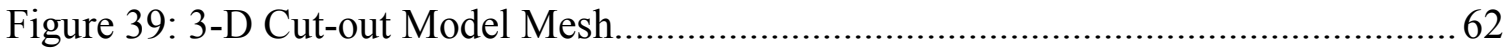


Figure 40: 3-D Cut-out Model Deflected Shape with Contours ..... 63

Figure 41: 3-D T-connection with Cut-out created with 20 Node Element...................... 63

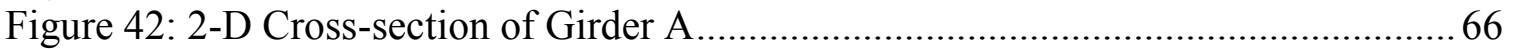

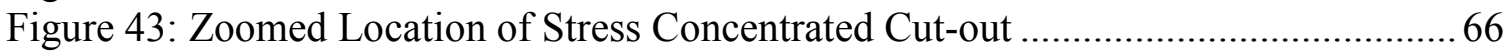

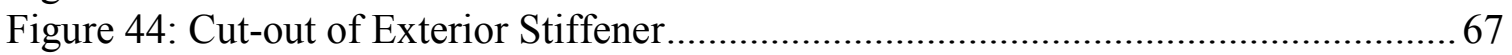

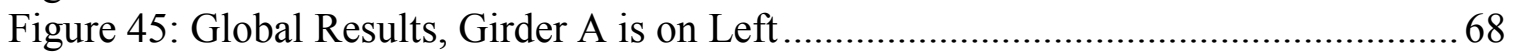

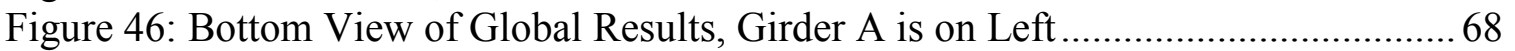

Figure 47: Fixed Boundary Condition with Push-Pull Diaphragm Side.......................... 71

Figure 48: Rotation of Top Flange Rest Fixed Diaphragm Side................................... 72

Figure 49: Actual Displacements on Cross Section with Push Pull on Diaphragm.......... 73

Figure 50: Modified Cut-Out ....................................................................................... 74

Figure 51: Stress Intensity Factor Case (Tada et al, 2000) ........................................... 76 


\section{List of Tables}

Table 1: Stress Intensity Factor Predictions, Finite Plate with Center Crack .................. 41 


\section{Introduction}

\subsection{Background}

Steel plate girders have been widely used in the United States for the construction of highway bridges. Plate girder bridges can occur either as simply supported spans or continuous spans. In many cases, including the ones of interest in the current study, the plate girders take the form of I beams, with two flange plates and a web plate. Several distinct configurations have been used, each with its own problems. Some large bridges consist of two plate girders, separated by cross-frames and supporting multiple floor beams parallel to the girders. Alternately, a common configuration consists of multiple parallel plate girders supporting a slab. The latter configuration is illustrated with a partial cross-section in Figure 1. In other cases, multiple plates are joined to form a closed box girder, with at least two web plates and top and bottom flange plates. Typically these are referred to as box girders, with the term plate girder used to describe I shaped girders.

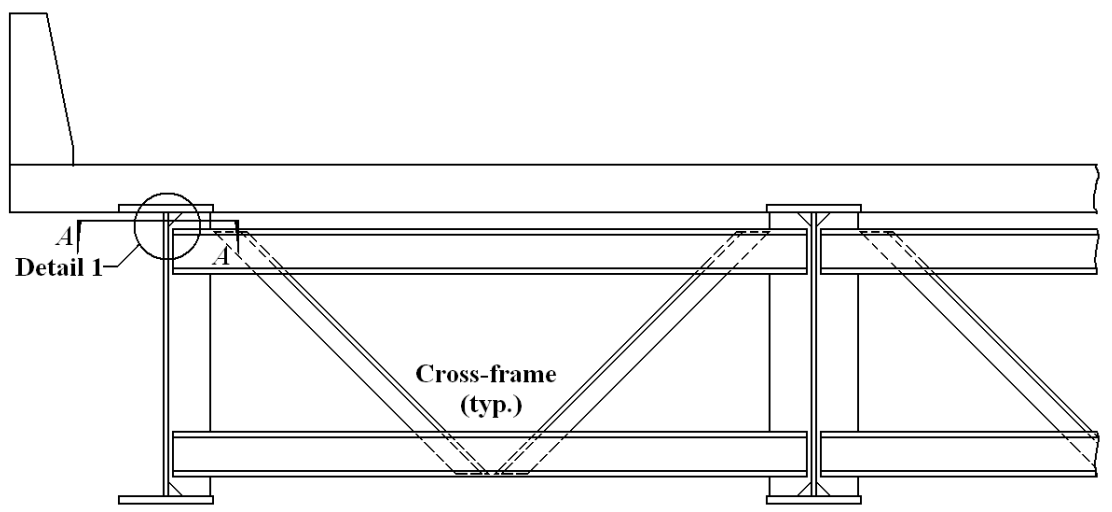

Figure 1: Multi-Girder Bridge with Cross-frames 
In plate girder bridges, cross-members are an essential part of the superstructure, whose exact function depends upon the specific superstructure design. In the case of a two girder design with multiple parallel floor beams, the cross members act as primary structural members, transferring loads from the deck slab through the floor beams to the longitudinal girders. In the case of multi-girder designs, such as the structure shown in Figure 1, the cross-members act as secondary members, coupling the multiple girders together to allow them to share the load transferred directly from the slab to the girders. The cross-members themselves may be either solid beams, or truss-like structures, such as the one shown in Figure 1, with the truss-like structure, called cross-frames, being more predominant in larger structures. In either case, the transfer of loads to the girders is accomplished through connection plates or connection angles. The cross-members may be either bolted or welded to the connection plates, but in recent years, the connection plates have been welded to the girders. This study is focused on welded connection plates in solid web plate girders.

It has been well known for many years that welded details in structures are prime sites for the development of fatigue cracks or fractures (Munse, 1964; Fisher, 1990), with recorded instances of failures extending back to at least 1900. Therefore, certain standard practices have been specified for design of multi-girder bridges to eliminate problems. It is known that transverse fillet welds in tension flanges are a prime site for fatigue crack development, and that tri-axial tension at the intersection of three perpendicular welded plates can lead to brittle fracture. To avoid these situations, a $1 / 2$ " to 1 " corner is typically cut-out at the top and bottom of the connection plates and stiffeners to prevent the triaxial tension problem (Fisher, 1990). Below the cutout, the connection plate is typically 
attached to the girder by fillet welds. Until recently, the connection plates were typically welded only to the compression flanges to remove possible stresses at any transverse welds on the tension flange that can lead to fatigue cracks (Stallings, 1997). However, it has been discovered that this detail allows rotation and out of plane bending of the web to occur causing locally high stresses near the tip of the weld in the web (Figure 2), and leading to a different but at least equally severe fatigue cracking problem. Upon occasion, larger web gaps than shown have been used, which will reduce the distortional stresses somewhat.

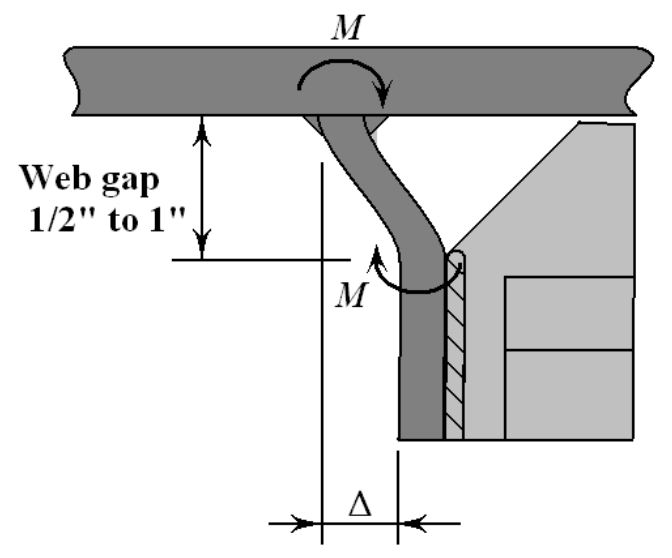

Figure 2: Detail 1-Web distortion at the web gap between the flange and the connection plate weld in a negative bending region

\subsection{Web Distortion and Distortion Induced Cracking}

Between 1978 and 1981, 60 out of 142 bridge sites studied developed out-of-plane distortion fatigue cracks. In plate girders, this occurs due to an immediate change in stiffness between the flange and stiffener (Fisher, 1984). Such distortion can occur at connection plates for either right or skewed bridges, but the situation may be exacerbated at skewed connections, where eccentricity can add a twisting component about a certical 
axis to the horizontal distortion, as shown in Figure 3. Currently, AASHTO does not have any provisions to help with this issue (Roddis \& Zhao, 2003).
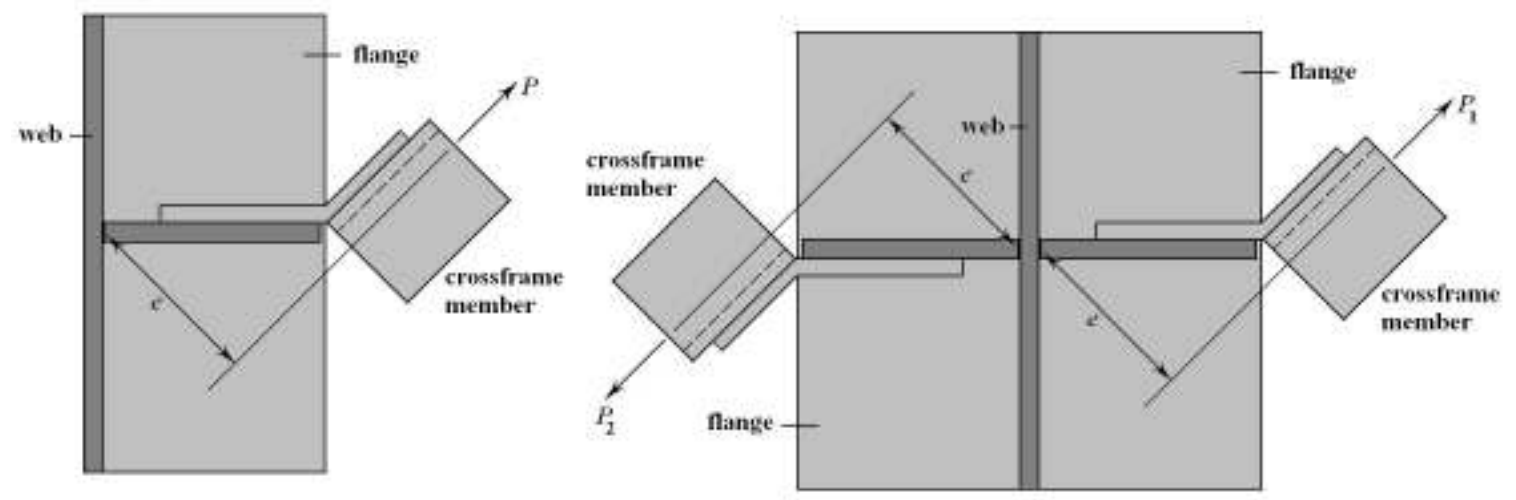

Figure 3: Sections at Skewed Cross-Frames

\subsection{Fatigue Analysis and Fracture Mechanics}

The fatigue life of a structural detail may be considered to consist of two stages: crack initiation and crack propagation (Schijve, 2001). If an initial crack does not exist, the majority of the fatigue life is expended in the crack initiation. During the crack initiation stage, the traditional stress-life (S-N) method is applicable for high cycle fatigue, and the Coffin-Manson strain-life approach is applicable to low cycle fatigue. Material surface roughness, surface damage, surface treatments, and the existence of dislocations in the crystal structure are the main components influencing the crack initiation period. If no singularities exist, such as sharp cracks, stress concentration factors may be used as practical analysis tools, and finite elements are a convenient tool for evaluation of these stress concentrations. 
In many actual structures, small flaws or microcracks may be pre-existing as a result of fabrication processes that may be considered to constitute an initial crack. In this event, crack propagation may constitute fatigue life. Once a fatigue crack has formed, the crack propagation stage and evaluation of the potential for brittle fracture are treated using principles of fracture mechanics (Schijve, 2001; Anderson, 1995). Three modes of crack deformation have been defined: opening in tension (mode I), in-plane shear (mode II), and transverse shear (mode III) as originally proposed by Irwin (1957). In complex details, a combination of these modes may need to be considered.

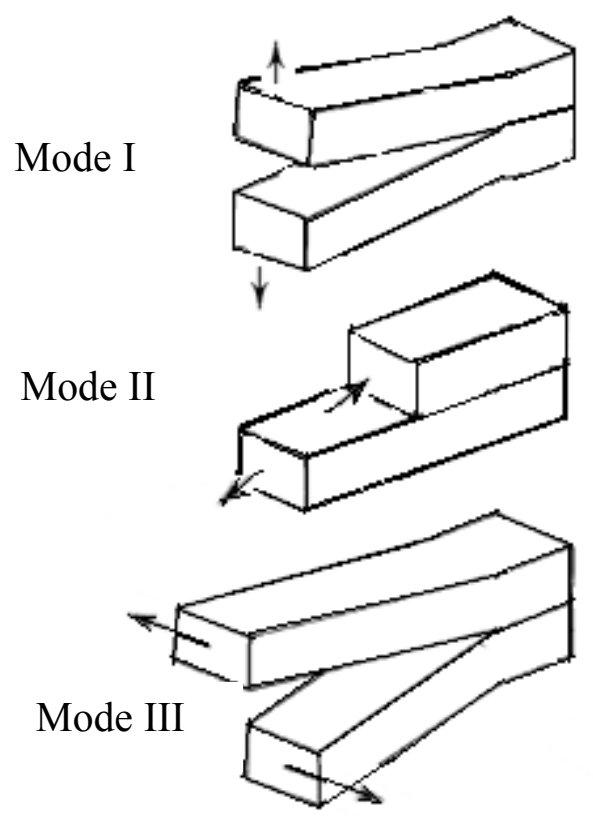

\section{Figure 4: Basic Modes of Crack Surface Displacements (Barsom and Rolfe, 1999)}

There is a stress intensity factor associated with each mode, and with different crack geometries. Stress intensity factors for a given geometry, and the critical stress intensity factors for a given material and operating temperature are required to predict crack growth rates and fracture strengths. They represent the severity of the stress intensity compensating for shape and load applied (Schijve, 2001). The size, shape, and 
orientation of the crack are all factors involved in determining the stress intensity factor (Fisher, 1984). Fracture mechanics has proven to be a valuable tool in evaluation of remaining fatigue life when cracks have been identified. Finite elements have also been used to evaluate stresses in the vicinity of cracks, but considerably more care is necessary in the presence of a crack because of the mathematical singularity in the stress field that exists at a crack tip.

\subsection{Case Study- The Carter Glass Bridge}

The Carter Glass Memorial Bridge on Route 29 over the James River is located in Lynchburg, Virginia. The first two lane bridge was built in 1949. A parallel superstructure added two more lanes in the 1970s. The Carter Glass Bridges is one example of a skewed bridge where distortion induced cracking has led to a significant web fracture. The girders of this bridge will be modeled to explore the stress concentrations and potential for cracking, to evaluate the efficacy of the global-local finite element modeling approach.

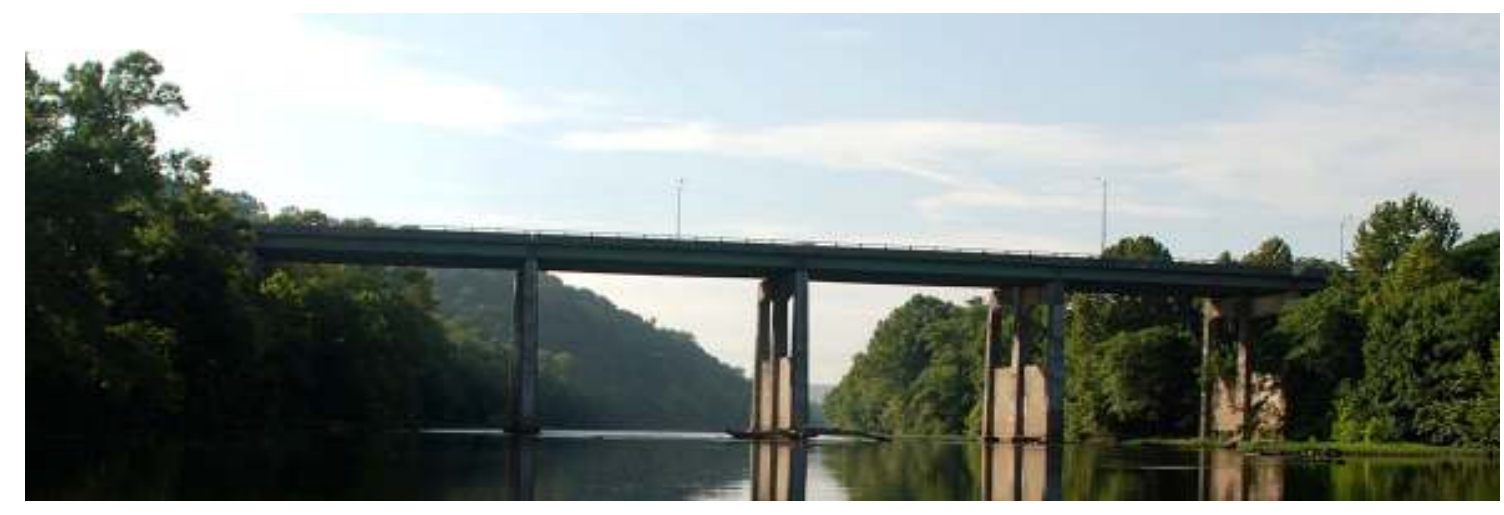

Figure 5: The Carter Glass Bridge, Lynchburg, VA

Figure 6 is an actual crack in the web caused by the distortion from the internal stiffener, shown from the exterior side, so the diaphragm is not visible. Girder A, where 
the crack is located, is located in the passing lane in the South Bound direction. It is apparent that the crack has progressed through the brittle fracture stage. This crack is the reason why girder A was chosen for the specific local model studied in this thesis. The observed fracture appears to have begun with a fatigue crack through the web that started near the tip of the fillet weld. A similar crack, which had not yet reached critical length, was observed to have propagated on the opposite side of the stiffener from the crack shown.

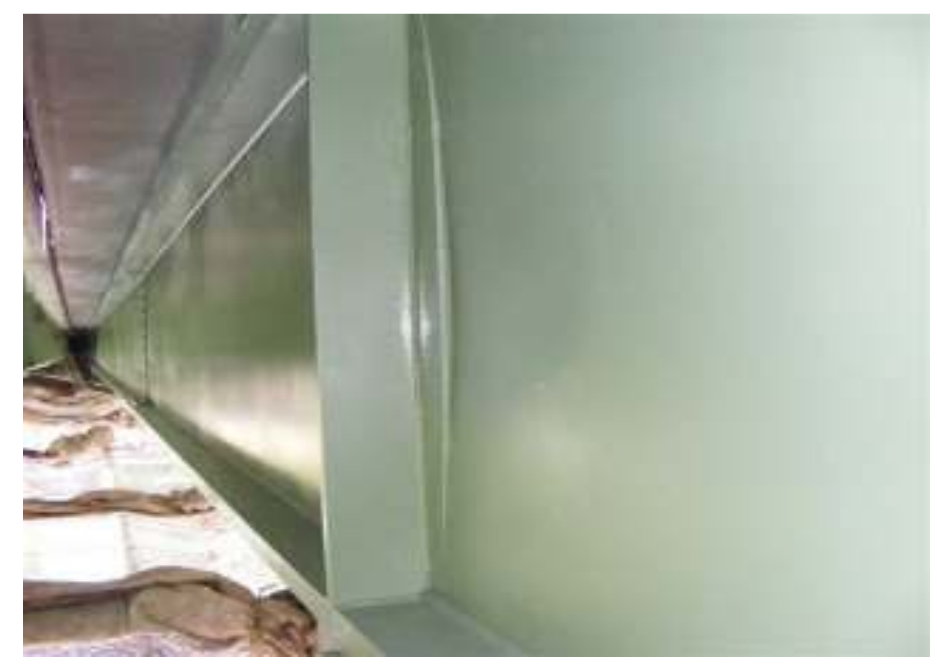

Figure 6: Web Cracking in Plate Girder 


\section{Fracture Mechanics}

Fatigue crack phenomenon in a material occurs on a micro scale and then on a macro scale. In the initiation period for a fatigue crack, the crack is not visible by the unaided eye. A fatigue crack starts by micro-cracks forming within the slip bands (Suresh, 1998). Cyclic shear stress causes a cyclic slip in slip bands, which leads to cyclic plastic deformation. A crack grows a minuscule amount after multiple cycles, because material properties do not change after a cyclic load (Alam \& Wahab, 2005). Cyclic stress intensity occurs at the crack tip due to a fatigue load on a cracked sample. The stress intensity factor measures the stress distribution around the crack tip. These fatigue cracks occur more easily on the grains near the exterior surface at stresses well below the yield stress.

There are several surface phenomena that effect the crack initiation period. The first is surface roughness, which involves defects from the heating and rolling process. Surface treatments can include nitriding, and shot peening. Although shot peening has sometimes been used for plate girders, it is not known whether this practice was used on the current bridge, so it is not a factor that is considered for this study. Surface damage, such as scratches, dents, and fretting, can form sites where crack initiation can occur in plate girders. Notches, holes and imperfections cause an inhomogeneous stress distribution.

Typical defects and cracks that may occur in a fillet weld are shown in Figure 7. The "stress concentration factor, $\mathrm{K}_{\mathrm{t}}$, is defined as the ratio between the peak stress at the root of the notch and the nominal stress which would be present if a stress concentration 
did not occur" (Schijve, 2001). The stress concentration magnitude depends on the shape of the notch. The root radius is an important variable for this factor. The initiation period gives way to the crack growth period once the microcrack has grown to the point where the free surface conditions no longer affect crack growth.

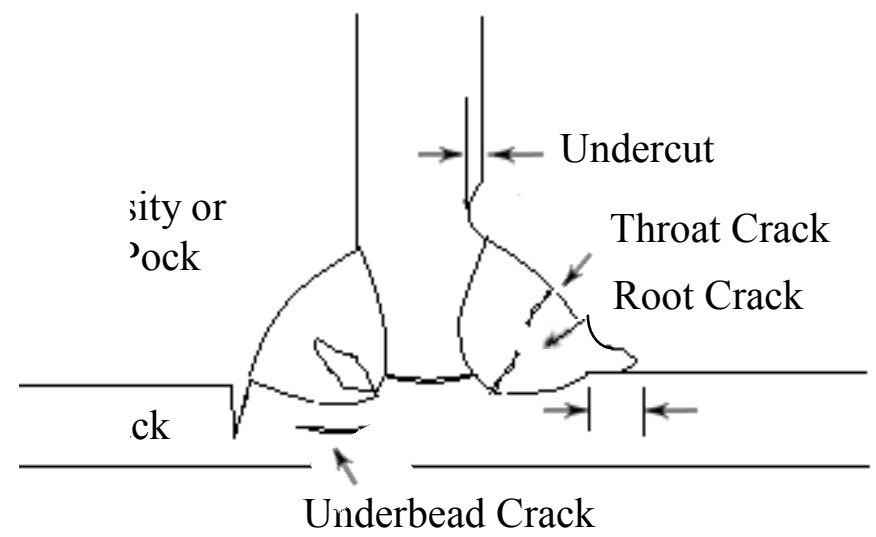

\section{Figure 7: Imperfections and Cracks in Fillet Weld (Barsom and Rolfe, 1999)}

Once a crack has developed, its resistance to growth depends upon the material bulk properties and environmental effects (Schijve, 2001). Fatigue crack growth rate depends upon current fatigue damage, crack-tip stretching, and associated material separation, as well as the nominal stress gradient in the vicinity of the crack tip. During the crack growth phase, the relevant residual stresses are crack tip stresses, which are perpendicular to the crack plane due to plasticity when no load is applied. Currently, the best approach to computing the effect of the crack tip residual stresses on the fatigue crack growth behavior has not been settled upon (Kujawski \& Stoychev, 2008).

From an analytical perspective, two types of cracks must be considered: part through and through cracks. Part through cracks may be corner cracks or surface cracks. Although the shape of such cracks can be complex initially, it has been observed that the crack fronts tend to grow into relatively smooth curves that can be idealized as partial 
ellipses as a result of the stress patterns that develop at the crack tips. A through crack can be idealized as an edge crack or a center crack. A through crack is usually considered as a two-dimensional problem, although three-dimensional effects can be present in some cases, and the most useful theoretical results are available for plane strain conditions. The geometry of the cracked specimen can be as simple as a twodimensional plate or complex three-dimensional geometry.

\subsection{Stress Intensity Factor}

The stress concentration factor is a dimensionless shape factor that accounts only for the geometry at finite notches and stress concentrations. In the limit, as the radius at the root of an elliptical notch goes to zero, the notch approaches a crack. If plane strain conditions exist, and the material remains linearly elastic, a mathematical singularity in the stresses occurs at the crack tip. The appropriate measure of the strength of the singularity relative to the far field stresses is the stress intensity factor. Unlike the stress concentration factor, the stress intensity factor is not dimensionless because it accounts for the applied load, and is not simply a scaling factor. For modes I, II, and III, the stress intensity factors are designated as $\mathrm{K}_{\mathrm{I}}, \mathrm{K}_{\mathrm{II}}$, and $\mathrm{K}_{\mathrm{III}}$ respectively. Under given temperature conditions and for a given elastic material, the limiting values of stress intensity factors at which fracture occurs are designated as $\mathrm{K}_{\mathrm{Ic}}, \mathrm{K}_{\mathrm{IIc}}$, and $\mathrm{K}_{\mathrm{IIIc}}$ respectively. For sub-critical crack lengths, the rate of crack growth has been shown to be a function of the relevant stress intensity factors, $\mathrm{K}_{\mathrm{I}}$ in particular.

The actual stress intensity factor is compared to the plane strain fracture toughness characterized by the critical stress intensity factors $\mathrm{K}_{\mathrm{Ic}}, \mathrm{K}_{\mathrm{IIc}}$, and $\mathrm{K}_{\mathrm{IIIc}}$. The plane strain fracture toughness is the highest value of stress intensity that the material can 
withstand without fracture. Therefore, if the actual factor caused by the loading exceeds that value, fracture will occur. In many cases, particularly for relatively tough, ductile materials, it is difficult to construct a specimen that allows direct measurement of the critical stress intensity factors (Barson and Rolfe, 1999). However, correlation of the critical stress intensity factor $K_{I C}$ with other widely used measures of fracture toughness, including the Charpy V-Notch test has been achieved. A Charpy V-Notch test can be completed to estimate the plane strain fracture toughness, $\mathrm{K}_{\mathrm{IC}}$. The plane strain fracture toughness is a temperature dependent material property. If a Charpy V-Notch test is conducted, the plane strain fracture toughness can be estimated by an empirical equation that uses the Charpy V-Notch impact energy (Brnic et al, 2010). This value can be used to estimate the critical crack length, provided the relevant stress intensity factor can be determined for the detail in question.

In a two-dimensional geometry with a through thickness crack, there is a transition from plane strain to plane stress in the crack tip region due to plasticity, which modifies the true stresses in the predicted singular stress-strain field forces (Bakker, 1992). For a plate with an elliptical crack, a correlation can be made between critical compression load and the stress intensity factor (Altus et al, 1992). When multiple cracks form adjacent to each other, they may produce a higher stress intensity factor. When a beam is subjected to a tensile axial stress perpendicular to the crack plane, the cracked beam cross-section is assumed to be equivalent to an elastic joint (Carpinteri, 1991).

Different equations are used to specify the stress intensity factor depending on crack location, the type of crack, geometry, and loading. The stress intensity factor for the mode I crack opening has the general relationship: 


$$
K_{I}=\sigma_{\text {nom }} \sqrt{\pi a} F(a / b) .
$$

The function $F(a, b)$ in equation [1] is a correction factor for the stress intensity factor that depends on the crack shape, free surface stress gradient, location of the crack, width of the plate that the crack is in, and the geometry of the crack front (Yazdani \& Albrecht, 1990). $\quad F(a / b)$ may also vary depending upon whether the applied loading causes bending moments about the local z-axis or tensile forces (Ferreira \& Branco, 1991). Most of the available closed form results have been obtained for a strip of infinite width. In these results, the ratio of the height from center of crack to the point of applied stress and half the width of the specimen is equal or larger than three (Tada et al, 2000). For a finite strip, the function $F(a / b)$ can increase the $K_{I}$ factor by as much as seven times that of an infinite strip in extreme cases (Patricio \& Mattheij, 2007).

For example, an edge crack in an infinite width plate has the exact solution $K_{I}=\sigma_{\text {nom }} \sqrt{\pi a}$, where $\mathrm{a}$ is the crack width. The factor is multiplied by 1.1215 if the plate has a finite width, Figure 8(a). In a semi-infinite plate with two edge cracks, Figure 8(b); the stress intensity factor is approximated as $1.122 \sigma_{\text {peak }} \sqrt{\pi a}$, which also equals $1.122 K_{I} \sigma_{\text {nom }} \sqrt{\pi a}$. These examples are for stresses applied at the infinite edge perpendicular to the crack direction (Schijve, 2001). 


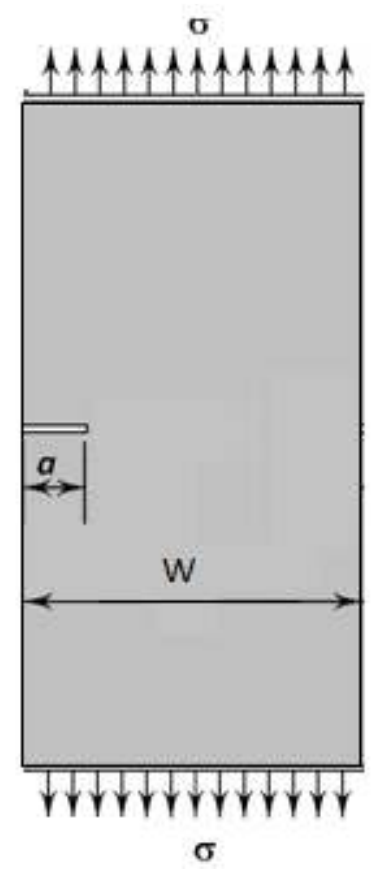

(a)

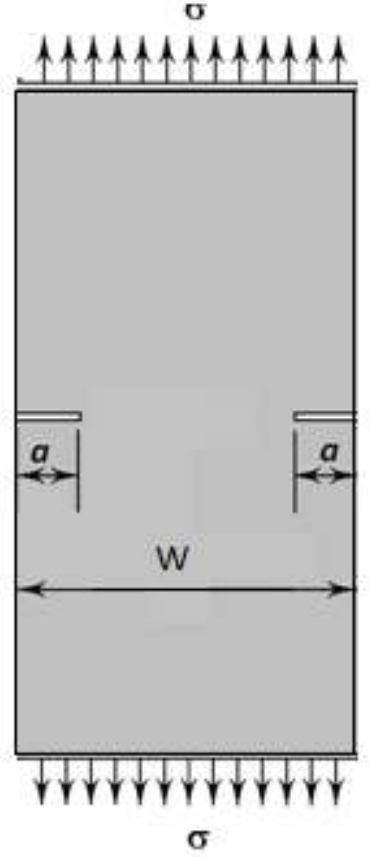

(b)

Figure 8: Semi-infinite Strip (Schjive, 2001)

For many practical configurations, closed form evaluation of stress intensity factors is difficult. In such cases, the stress intensity factors must be calculated using numerical approximations, such as finite-element software. Several techniques have been developed to either model the singularity at the crack tip, or to perform domain type calculations such as $\mathrm{J}$ or $\mathrm{M}$ integrals that provide equivalent information (Alam \& Wahab, 2005; Miranda et al, 2003; Chang \& Wu, 2011).

Unfortunately, most of the known equations for the stress intensity factors use planar stress/strain. For example, the Lafayette Street Bridge had a crack in a girder web at lateral gusset plate and transverse stiffener web gap. When the crack started, it was a semi-elliptic crack in a weld corner. The stress intensity factor could be analyzed at that point and then again once the crack is a through crack in the web (Fisher, 1984). 
In addition to predicting the occurrence of fracture, the stress intensity factor is a key parameter in the crack growth rate (Schijve, 2001) which is required to estimate the remaining life given the current crack length. Fatigue crack growth rate is a function of the stress intensity range, which is the difference between maximum and minimum stress intensity. Paris, Gomez, and Anderson first proposed this relationship in 1961. The fatigue crack growth process was broken into three regions of growth behavior, with the original crack growth equation only applying to region II. Priddle proposed an empirical relationship to represent crack growth in all three regions. McEily then developed an equation, also intended to represent the entire crack growth process, that was based on a physical model (Massarelli, 1997). McEily's crack growth equation takes the form

$$
\frac{d a}{d N}=C\left(\Delta K-\Delta K_{t h}\right)^{2}\left(1+\frac{\Delta K}{K_{c}-K_{\max }}\right)
$$

In this equation, $\Delta K$ is the stress intensity range, $K_{c}$ is the critical stress intensity, $K_{\max }$ is the maximum stress intensity, $\Delta K_{t h}$ is the threshold stress intensity range, and $\mathrm{C}$ is a material constant.

Typically, the development of the curved fatigue crack path and its stress intensity factors are calculated using finite-element software, using small crack increments. A common feature in finite element analysis of crack tip growth is the use of automatic remeshing algorithms and crack tip elements (Miranda et al, 2003). This process is completed, because the stress intensity factors vary along the crack front. 


\section{The Global-Local Modeling Approach}

Evaluation of the stresses near a fatigue critical location, such as a crack, or other severe stress raiser can only be performed analytically for certain special cases where the geometry is relatively simple. As the complexity of the region's shape increases, it becomes necessary to resort to numerical modeling procedures, such as the finite element method. It has been noted that highly refined meshes are required for determination of stress intensity factors (Schijve, 2001). In the simplest cases, plane strain models can be used, but for more complex regions, it may be necessary to use three-dimensional discretization. This type of discretization may lead to a very large model, just for the small region of interest. Such a model may be referred to as a local model, since it only seeks to model the small region near the detail of interest. In order to properly apply such a local model, it is necessary to obtain boundary conditions that replicate the region's true response as part of a larger structure. This leads to the global-local modeling approach.

The overall strategy of global-local modeling is straightforward. As a first step, a global model of either the entire structure, or a sufficiently large portion of the structure that any boundary condition errors do not significantly affect the displacements, strains or stresses in the small region of interest must be constructed. The output of the global model is then used to provide complete or partial boundary conditions to the detailed local model of the region of greatest interest, to permit the additional mesh refinement needed for accurate prediction of local stresses. Hence, the global model must be sufficiently refined to provide reasonably accurate information at the boundaries of the 
local model. What this means may be quite varied, depending upon the specifics of the problem of interest, and the nature of the local model.

\subsection{Global Modeling Options}

In its most common usage, the phrase "global-local model" refers to a hierarchy of finite element models, where both the global and the local models are true finite element models. This approach is not always strictly necessary, when the global behavior can be extracted from a simpler model. For example, Roddis and Zhao (2003) were able to use a relatively simple truss analysis for the "global" model in analyzing the distortion induced stresses for a two girder system with multiple floor beams. This simplification was possible because the cross-trusses were primary members in the load carrying system, and it was relatively straightforward to determine the loads transmitted to the girders through the cross-frames. DePiero et al (2002) used a global truss and beam model to obtain the input to two- and three-dimensional local solid finite element models, because the structure under consideration was a truss structure. By contrast, Aljutali (2007) examined stresses in a region adjoining twin box girders, and found it necessary to analyze the entire superstructure, using plate elements for the global model and solid elements for the local model. At the other extreme, both global and local models may be solid three-dimensional models, in which case the local model may be considered a substructure model embedded within the global model. This case is in some ways particularly simple and attractive, since the large number of internal nodes within the local model can simply be condensed out during the global analysis, with the 
substructure response calculated subsequently by back-substitution after the global response has been calculated.

In many multi-girder bridges, such as the Carter-Glass Bridge, the cross-frame or diaphragm members are secondary members that do not directly transfer loads from floor beams to the girders, but are loaded indirectly through the girders themselves. The functions of the secondary members comprising the cross-frames or diaphragms are then to help distribute vehicular loads between multiple girders, to brace the girder system against lateral instability during construction, and to provide wind bracing during the service life. Since the loads transmitted between girders depend upon both the relative displacements of the girders and the details of the diaphragms or cross-frames, simplified approximate global models are generally not suitable; and it is necessary to construct a global model that represents the full complexity of the structure. Even in this case, a number of choices are available for the level of refinement of the global model. It may be possible to model the multi-girder system using beam elements only, with an offset from a plate model used for the slab, if the diaphragm consists of solid beam members. The local bending moments and displacements within the intersecting girders and diaphragm members can then be used to construct boundary conditions for the local model. Some additional refinement is needed for the girder model if the diaphragm consists of a discrete cross-frame. Tedesco et al (1995) discretized the girders and the slab using plate elements, and modeled a solid diaphragm member as a beam element connected to the girders using rigid links. Although they were not interested in constructing a local model, Chung et al (2006) modeled the girders and connecting discrete cross-frames in ABAQUS using beam elements and rigid links. It is likely that 
this model would capture global response adequately, but would probably not provide satisfactory input to a local model. A variation on this approach is to use plate elements for beam webs, and beam elements for the beam flanges. This approach seemingly reduces the number of degrees of freedom, while still capturing the beam depth to provide attachment points for a discrete cross-frame, but it is not clear how much model size reduction is actually gained, and spurious results could arise from displacement incompatibilities between beam and plate elements. It is also not clear that the boundary conditions needed for a detailed local model could be adequately determined.

Several researchers at the University of Virginia have utilized superstructure models of multi-girder bridges that are composed entirely of plate elements, an approach that has been largely successful, particularly if the girders are connected to the slab using deformable haunch elements instead of rigid links (Tilley, 2004; Simons, 2005; Lydzinski, 2006). Most recently, Rostek (2010) used ABAQUS plate elements to discretize the entire superstructure of a three span section of the Carter Glass Bridge, an approach that should provide adequate information for the boundaries of a local model. He also considered a very simple local model using plate elements only, to determine whether high bending stress regions of the plate could be identified near the connection plates, but he did not attempt to investigate the local stresses at the connection fillet welds, which would require solid element modeling, and he did not attempt to construct a mapping from the global model to the local model boundary conditions. For the current work, it was decided that Rostek's model contains sufficient refinement that it can be used as the global model for a solid element local model, provided the boundary conditions are carefully constructed. 


\subsection{Partial Local versus Complete Local Modeling}

Regardless of the level of discretization, or the detailed form of the global structural model, the results of that model will often be unable to provide the detailed stress and deformation information required at the location of severe local stress gradients. This is particularly likely when the scales between the structure and the local regions of interest vary widely. In this case, a separate local model can be constructed, with the output of the global model used to "inform" the local model. The level of information provided to the local model distinguishes between complete and partial local modeling.

A local model only represents a small section of the structure where details of interest occur that cannot be adequately represented in the global model. In order for the local model to provide useful results, it has to be provided with input obtained from the global model. For a fatigue/fracture problem, the local model is needed to predict the driving force for crack initiation and growth. It is possible to distinguish between two levels of local models, which are referred to herein as complete local models and partial local models.

A complete local model uses all information provided by the global model at the boundaries of the local model as input. For example, the complete displacement field of the global model at the boundaries of the local model can be used as the "loading" to the local model. A special case of this is the case where the global and local models use the same element types, so the local model may be treated as a substructure, or "superelement" within the global model. More generally, the global and local models may use different element types that are appropriate to the level of detail desired in the results. 
For example, a global model using beam elements and a local model using solid elements could be satisfactory for a multi-girder bridge with a beam type diaphragm, provided an adequate boundary condition mapping between the two models can be established. Alternately, the girders and diaphragms (or cross-frames) could be modeled with plates in the global model, and two- or three-dimensional solid elements used for the local model. When there is a mismatch of element types between the global and local model, or if there is a mismatch of mesh refinement between the global and local models at the boundaries of the local model, the global model must be properly interpreted in order to provide suitable boundary conditions for the local model. Even so, some spurious results may occur in the elements very near the boundary of the local model as a numerical artifact of the boundary condition mapping.

By contrast, a partial local model does not attempt to establish a complete set of boundary conditions from the global model. As an example, Roddis and Zhao (2003) only extracted the force in the cross-frame members from the global model, and idealized the displacement boundary conditions at the ends of the local model as fixed. A partial local model also may not attempt to portray the full geometric complexity of the local region of interest. For instance, DePiero et al (2002) considered both two-dimensional and three-dimensional models of a crack prone angle connection. Rostek (2010) only examined the local stresses in the web of the Carter Glass Bridge at the plate level, and examined the stresses under a nominal, as opposed to actual loading. Frequently a partial local model replaces the actual boundary conditions, at the boundaries remote from the detail, with fixed boundaries and applies the actual load or some nominal equivalent. It is the simplest way to construct a local model and therefore is widely used. The 
consequences of such an incomplete idealization have not been fully examined, but some unanticipated results may occur if the contributions of the far field, global stresses are not correctly represented.

\subsection{Global- Local Approach for the Carter Glass Bridge}

\subsubsection{Global Model}

A global model of the Carter Glass Bridge previously was created by Rostek (2010) using ABAQUS. In his study, he modeled the entire superstructure, including girders, cross-frames and slabs using plate elements. The superstructure model provides a satisfactory representation of overall response, but is insufficiently refined to predict the localized stresses introduced at the fillet weld tips in the web. As a part of his study, he examined local stresses caused by plate distortional bending, but the plate elements used were incapable of predicting stresses near fillet weld ends in the web, which is the most likely site of crack initiation. The stress concentration and stress intensity factors were not the emphasis of Rostek's study. Although the locations of interest in the global model were not modeled in sufficient detail for the current study, it is believed to provide an adequate global model. One concern with Rostek's model is that the global model does not include a gap in the stiffener between the connection plate and the top of the web when modeled, so that using displacements at the boundaries of the local model might not allow the anticipated distortion to take place.

\subsubsection{Local Model}

For the current study, it was decided to utilize the commercial program ANSYS, because of the versatility afforded by the ANSYS Parametric Design Language (APDL) for model creation. The connection plate region of Girder A was modeled twice in 
ANSYS; once with an additional fillet weld connecting the stiffener to the tension flange, to investigate whether such a weld would alleviate the high stress concentration at the end of the connection plate to web fillet weld. The models were created using APDL with parameters allowing easy edits and use for multiple bridges. The objective is to use the models as a building block for subsequent analysis of multiple skewed bridges with different angles of skew, and girder dimensions. The models were created by key points, then areas, then volumes using APDL. A solid 10 node tetrahedral element was used for all elements to obtain superior convergence. Smart size was enabled to create a fine mesh without too much refinement. The active size level was a one, which is the finest mesh, and a scaling factor for the computed mesh sizing was 0.2. This allowed an element refinement only to be needed at the four roots of the notch on the tension flange side. A level three refinement, middle refinement, was used at those locations. Figure 9 shows the element view of the completed local model.

A segment of the girder with a length of 42.5 inches was modeled, which extends halfway between the connection plate and the adjacent stiffeners on either side. The stiffener and connection plates were centered in the model. The angle was represented with a length of five inches; this was cut off right before the additional angle cross frames. In addition, several simplified two- and three-dimensional models were created of the region along the fillet welds connecting the connection plate to the girder web to allow various aspects of the stress field to be considered within a simplified loading scenario. 


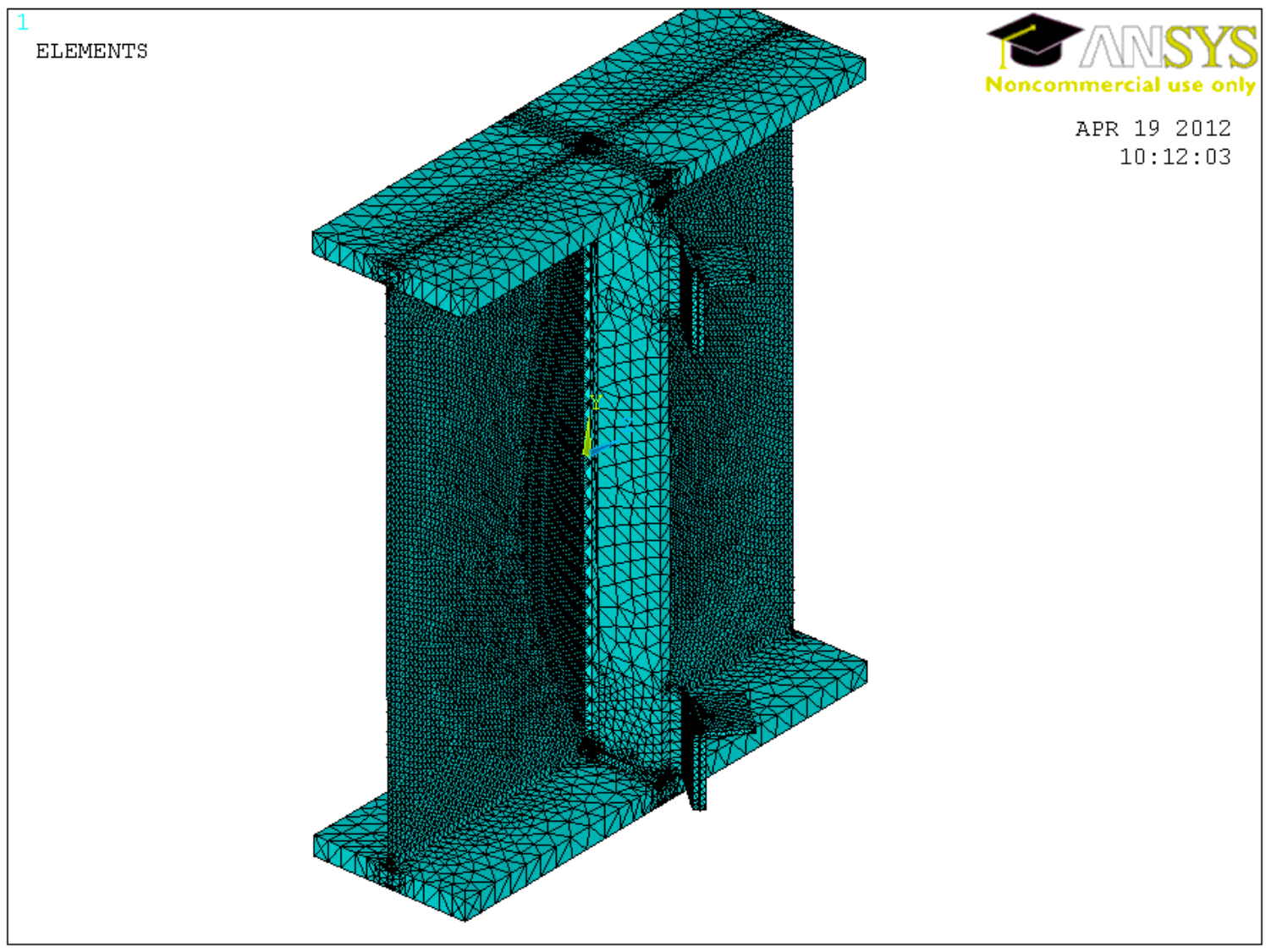

Figure 9: Local Model- Girder A

\subsubsection{Boundary Conditions}

After the model was completed; boundary conditions were created using Excel and MATLAB. For the complete global-local model, input conditions were taken from ABAQUS. Rostek's global model was created using center line plate elements. Therefore, a mismatch exists between the nodal displacements of the global and local model. The node displacements and rotations had to be interpolated to the node locations in the ANSYS model. A MATLAB code was written to use a Newtonian polynomial interpolation scheme and find the values along the center lines. Extrapolation was used to find the values for the rest of the nodes. Values for all nodes on the both plate girder 
cross-sections, along the cross-section of the angles, and top of the top flange along the length were evaluated.

Input boundary conditions might be inaccurate for several reasons. There were some bearing stiffeners missing in the global model. The center line of the flanges of the girders was located at the end of the web instead of the center line of the flange itself. The significance of this error is considered to be relatively small, given the overall depth of the model. The angles were modeled at equal distance from the center of the plate girder, which is a simplifying approximation that is not quite correct.

\subsubsection{Local Models used in the Study}

A global model of the Carter-Glass Bridge was previously created in ABAQUS, but only limited local results were obtained, using plate elements and highly idealized boundary conditions that do not reflect the true deformation and loading. The current study will focus on local connection models of the same bridge. Currently, there are cracks located at pier 10 at a skewed exterior connection. This location can be seen from the bridge plans, shown in Appendix 8.3. This observed fatigue crack is the major focus of modeling.

There were multiple objectives for this study. After a series of simplified twoand three-dimensional models were considered to evaluate the capability of ANSYS to represent stress singularities and evaluate stress intensity factors, more detailed threedimensional local models were constructed for the skewed diaphragm Four local models were constructed in ANSYS; skewed exterior joint, skewed interior joint, non-skewed exterior joint, and non-skewed interior joint. These models were developed using parametric dimensions, allowing it to be used for multiple bridges. The detailed studies 
herein focus only upon the skewed exterior joint. This is intended so that the CarterGlass Bridge can be considered as an example of cracking that occurs on skewed bridges. Initiation and propagation of cracks occurs at high stress locations, also known as hot spots, as well as meeting other fracture mechanic criteria. Therefore, locations of hot spots on all model schemes will be investigated. Figure 10 displays different locations of hot spots that may occur depending on loading and penetration. These hot spots are important in locating critical sites, and evaluating the probability of fatigue crack growth. As discussed in Chapter 2, an important measure of the importance of a location is the stress intensity factor. Unfortunately, few results are available for complex threedimensional geometry

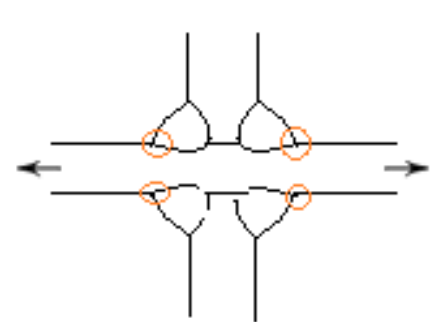

Partial Pentration Fillet Welds

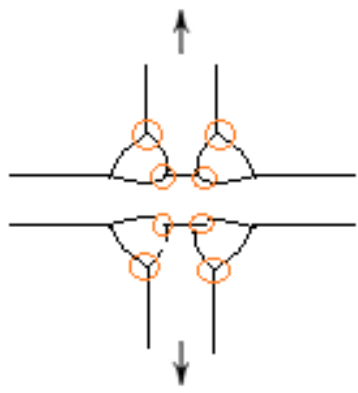

Partial Pentration

Fillet Welds

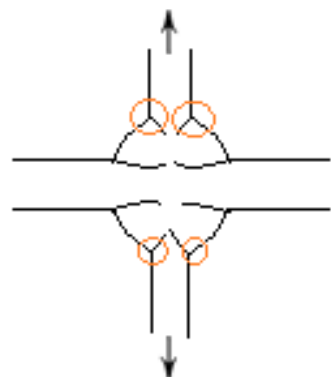

Full Pentration

Fillet Welds

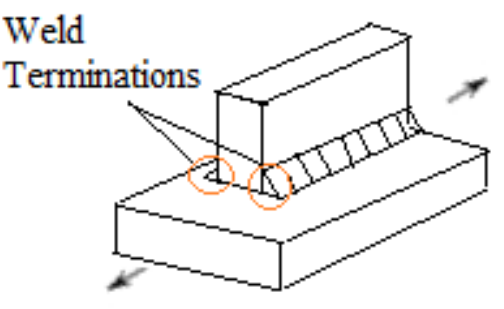

Partial Pentration Fillet Welds

Figure 10: Stress Concentration Regions in Fillet Welds (Barsom and Rolfe, 1999) 
Boundary Conditions are vital in finding accurate information for the goals stated above. Currently, several studies are available that have used a partial-local modeling; this involves using fixed boundary conditions instead of actual boundary condition. For the present study, the actual boundary conditions were taken from the outputs of the previously created global model. Stresses computed using both a partial-local and a fully-local model were then compared. The goal was to determine whether this partiallocal modeling technique is acceptable as an alternative to complete local modeling for the given configuration.

\subsubsection{Limitations in ANSYS}

After analyzing the models, it became apparent that ANSYS has some limitations for the type of modeling emphasized in the study. According to the ANSYS manuals, the program has a singularity and fracture mechanics capability. This feature was favorable for the purposes of the study. The two- and three-dimensional quadratic elements in the program have the capability of representing $1 / \sqrt{r}$ singularities, using the approach pioneered by Henshell and Shaw (1975) and Barsoum (1976, 1977). These features are incorporated into the program, but unfortunately their implementation did not appear to be particularly effective and it was not easily implemented in general three-dimensional models. In general the models are most conveniently formulated using key points, lines, areas and volumes. However, to implement the singularity features of the elements, it is necessary to create the model using nodes and elements directly. The mid-side nodes then have to be moved to the quarter points to implement the singularity. The same procedure is also possible in both two- and three-dimensions. However, in three dimensions, moving the nodes and creating the elements is even more complicated, 
making the feature difficult to use for complex geometries. It was found that refinement of the mesh near the location, while not reproducing the singularity, produces better stress results near the crack tip than explicitly introducing the singularity. A specialized pre- and post-processor specifically targeted toward analysis of cracks and fracture type problems might alleviate this problem, but was beyond the scope of the thesis.

ANSYS utilizes two different procedures to evaluate fracture potential. The first approach is based upon modeling the singularity using the crack tip elements, and using the KCALC command to evaluate the stress intensity factor. This feature is only available for planar conditions. KCALC uses displacement extrapolation during the postprocessing section. Alternately, following an approach developed by Shih et al (1986), the $\mathrm{J}$ integral is evaluated numerically on a path around the location of the singularity, using the CINT command. CINT should evaluate the stress intensity factor using the interaction J- integral approach during the solution phase. This command can also evaluate the J-integral calculation and the direct energy-release rate calculation. This information indicates that the CINT command should be used for problems with complex geometry and loading. This feature in ANSYS is complicated to use for even relatively simple three-dimensional geometries, and given the complexity of the region of interest for this problem, it was not considered to be feasible to use in the current study.

\subsubsection{Modeling Approximations}

Even within the local model, it is necessary to introduce some simplifying approximations to make the problem tractable. The end of the fillet weld of the bearing stiffener and connection plate to the web plate near the tension flange is the expected location of a critical stress concentration, both based upon available literature such as 
Fisher (1984) and from the photographs of the fatigue cracking observed on the Carter Glass Bridge. Cracks did not appear to originate in the longitudinal fillet welds joining the web to the tension flange. Therefore, it was decided that detailed modeling of the fillet welds on the plate girders connecting the web to the flanges was unnecessary. Thus the flange interfaces were modeled as continuous materials, and the fillets were not modeled. This simplified the model construction considerably, without sacrificing much useful information.

In modeling the fillet welds between the connection plates and the web and between the bearing stiffener and web, another assumption was introduced concerning the size and penetration of the welds. The exact shape of a fillet weld is only known if it is actually measured in the field. For modeling purposes, it was assumed that all fillet welds are triangular in shape. In reality, the shape is either concave or convex, and may be expected to be a function of the position along the weld. Also, the weld penetration was not specified explicitly on the available set of drawings. It was assumed that there was no penetration of the weld into the stiffener or connection plates, so the welds end flush with the surfaces of these plates. Finally, it was assumed that the fillet welds end precisely at the cut off or notch in the stiffener and connection plate. Since any imperfections in a weld are highly site specific, and will vary between even two nominally identical welds, it was also assumed that there were no imperfections in this welded joint. The ends of the weld are likely sites for imperfections, so a refinement of the model might include such imperfections.

Construction plans from Virginia Department of Highways from 1972 and pictures from the site were used to model all the connections. The cut-out dimensions of 
the stiffeners and connection plates at the top of the web were not given explicitly on the plans. Therefore, the dimensions were scaled off a photograph taken at the site by comparing the measured size of the notch in the photograph with the known width of the stiffener. Based upon these measurements, three quarters of an inch was used for both sides of the cut-off.

For negative bending, at the skew connections, the plans stated that the stiffeners were the same size as half of the flange width. If this is the case, the combined width of the stiffener plus the web thickness would exceed the width of the flange and the stiffeners and connection plates would overhang the edges of the flange. The site photographs did not show an obvious overhang, so it was assumed that the stiffeners only in the negative bending region would be terminated flush with the outside of the flange. It was felt that any error in this approximation would have negligible influence on the results.

\subsection{Preliminary Simplified Models}

Several different preliminary models were created before the detailed threedimensional model of Girder A was constructed. These models served to provide insight into modeling in ANSYS, with a particular emphasis on identifying potential regions of interest for stress concentrations in fillet welded joints as well as to learn the limitations of modeling in ANSYS.

\subsubsection{2-D Model}

Before attempting to model the problem of interest, it was decided to model a simple problem for which available solutions already exist. Thus, a simple finite width rectangular plate with a center crack was created first. Specifically, the plate was 
assumed to have a half width of 3 inches, with a half crack width of one inch. A finite length segment was loaded perpendicular to the crack orientation. The plate displays symmetry about the both the horizontal and vertical centerlines of the crack, so only a quarter of the plate had to be modeled. A $10 \mathrm{ksi}$ tension pressure was applied perpendicular to the existing crack. This case was evaluated with a relatively coarse grid of rectangular elements and again with automatic re-meshing. It was also assessed by using triangular elements only at the crack edge. Four- and eight-node planar elements were used. When using the eight-node elements, it was attempted to create a singularity by moving the mid-nodes to quarter points, in order to evaluate ANSYS's capabilities to predict stress intensity factors.

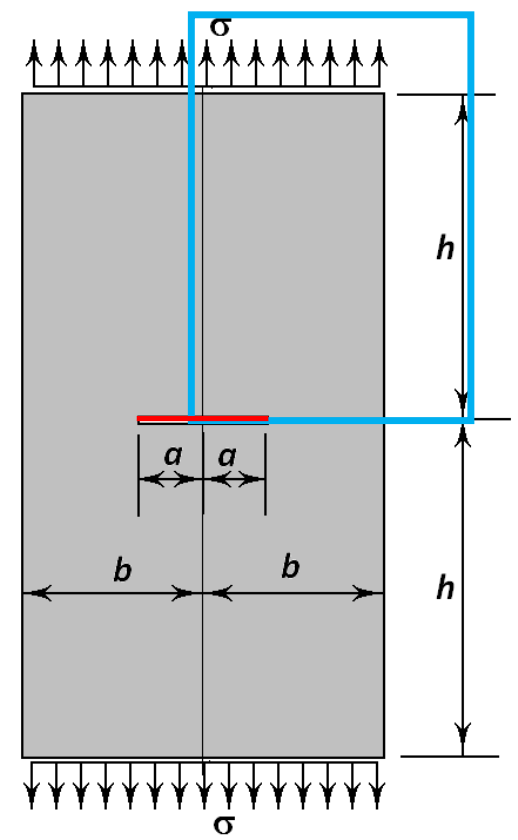

(a)

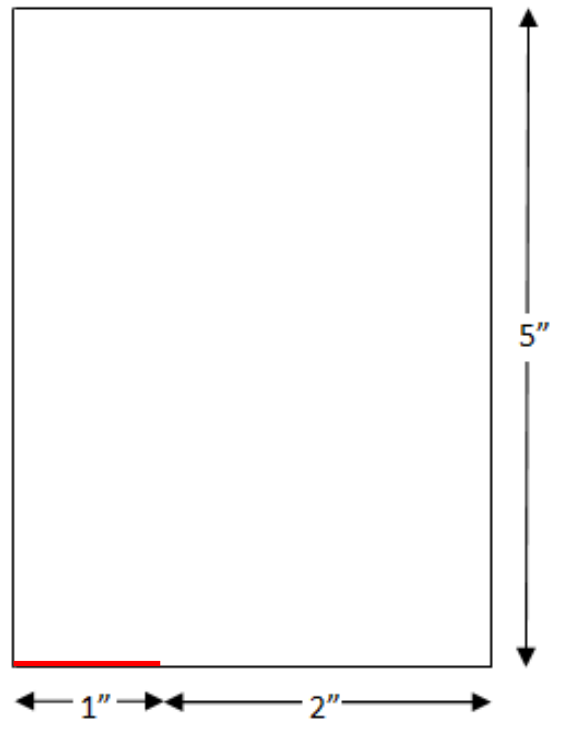

(b)

Figure 11: Plate Model (a) full model (b) section modeled, crack displayed in red

Once ANSYS's capabilities to handle a simple problem with a known solution had been explored, a simple fillet welded T-connection was created. This was analyzed 
to provide insight into the stresses that might occur in a simple connection that has similar geometry to the joint that was to be modeled, but without the three-dimensional complications. Two perpendicular plates were created with a fillet weld connecting them as shown in Figure 12. A gap was placed between the two plates, as in the actual connection. The $10 \mathrm{ksi}$ tension pressure pulled on the vertical plate. Four different sets of boundary conditions were used for the horizontal plate, in order to explore the influence of different degrees of transverse restraint of that plate in Chapter 3.

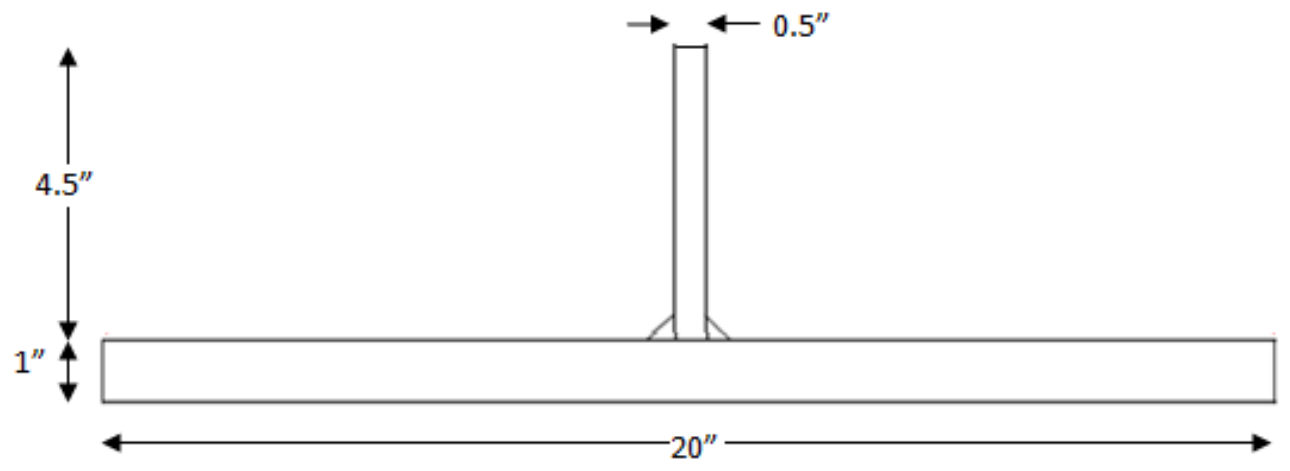

Figure 12: 2-D T-connection Boundary Conditions

In the first model, the ends of the horizontal plate away from the vertical plate were fixed (Figure 18). This model attempted to capture the stress transition if the horizontal plate is free to move transversely at the point of the T-connection. In the second model, the back of the horizontal plate was constrained against vertical motion, to evaluate the stress transition if the horizontal plate is not free to move transversely at the T-connection. The other two conditions were to compare the stress concentration for different boundary conditions. The size of the elements, type of elements, and methodology was modified as well. These modifications were the same for all the boundary conditions. Multiple levels of refinement were completed as well. 
In constructing the T-connection model, a small gap of 0.001 inches was included between the horizontal and vertical plates, to permit a model of an internal crack to be included, at the root of the fillet welds. As in the three-dimensional models, it was assumed that weld penetration into the vertical plates was negligible, a worst case scenario. It was assumed at the outset that this location is where the largest stress concentration would probably occur. The results of the numerical studies, along with subsequent research into similar studies suggest that this assumption is probably incorrect, and that the toe of the weld is a more likely site of crack origin. Several authors, such as Ferreira and Branco (1991), have indicated that the crack will initiate at the toe of the weld, rather than the root. This site of crack initiation is likely for several reasons. The geometry of the toe tends to cause a stress concentration even if no flaws exist. It is not unusual for flaws that can act as initial cracks to occur at the toe. Finally, the welding sequence tends to introduce significant tensile residual stresses at the toe. The presence of these residual stresses at the toes tends to cause the root to be in compression. Therefore, even if the fillet weld is not full penetration, the "crack" at the root will not tend to be in tension, and the highest concentration of tension stresses will tend to be at the toe. The T-connection model was altered to verify the results of that paper. The gap was removed and the same boundary conditions were tested. As in other studies, residual stresses were not included, but could be simply added to the calculated stresses, as long as the yield stress is not exceeded.

\subsubsection{3-D Models}

Once the two-dimensional models were understood, a couple of simple threedimensional models were created before the full local model was constructed. The goal 
was to evaluate the best way to obtain and observe accurate results before investigating the full local model, which has significantly more complex geometry. The first simplified three-dimensional model used an extruded two-dimensional T-connection. The force applied along the vertical plate was changed to affect more accurate representation of loading in the larger model. The majority of the loading was biaxial, tension pressure and along the depth of the connection. For the second simplified threedimensional model, the horizontal plate was extended past both ends of the vertical plate, as shown in Figure 13. The three vertical sides of the horizontal plate A, B, and C were then fixed. The vertical plate was pulled toward the free edge with a load of 20 kips divided between the four top corners of the vertical plate. Since the major focus of the stress concentration is at the gap in the stiffener, a cut-out was also added to the extended T-connection model. The boundary conditions were the same. The free end was on the opposite side of the notch at D.

The results of numerical studies conducted using the several simplified models, together with the complete local model, are presented in Chapter 4. 

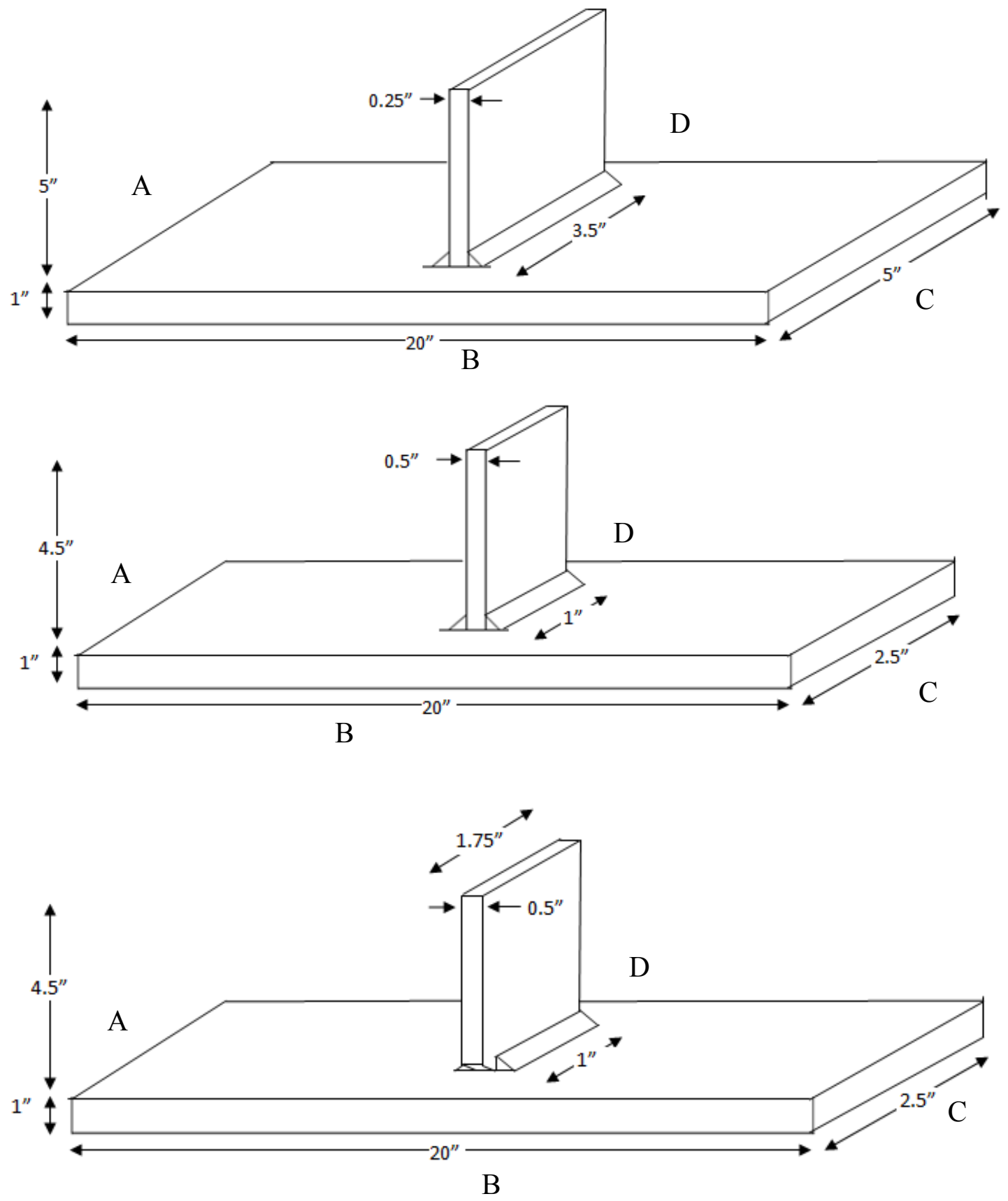

Figure 13: 3-D T-connection Dimensions for 8 Node Element, 20 Node Element, and Cut-out Model respectively 


\section{Results}

\subsection{Preliminary Model Studies}

The geometry of the three-dimensional regions around the girder/connection plate region is deceptively complicated to construct. Initially, it was not clear what steps were necessary to ensure that volumes that shared a side act as separate volumes. Simply specifying two areas sharing common key points did not suffice, since ANSYS simply made them the same area. The resulting volumes acted as if they were continuous material. This caused problems at the interfaces where full continuity does not exist, such as the interfaces between the connection plates and the tension flange, where no weld is present, and between the connection plates and the web, where full weld penetration was not assumed. When forces were applied, no gaps between the materials were observed, and no cracks or stress concentrations were present. It was decided to construct several simplified two- and three-dimensional preliminary model in order to develop an understanding of the available options for modeling the geometry of the fillet welds/connection plate/web properly as well as to gain some preliminary insight into the available techniques for evaluating stress intensity factors and the likely locations of regions of stress concentration in the complete model. The simplified models also permitted several options for material separation/crack introduction, without the complexities of the complete three-dimensional model. The overall goal was to find hot spots, how to model cracks using the capabilities of ANSYS, and the size of elements required to obtain convergence. 


\subsubsection{2-D Plate Model}

The first model investigated was a simple two-dimensional finite width plate with a center crack. This model was created using an eight-node element, and subsequently refined using six-node triangular elements. The purpose of the model was to investigate the capability of ANSYS to reproduce the known solution, and to consider the degree of mesh refinement needed for reasonable solutions to be obtained. Lesser purposes of the model were to investigate the operation of the KCALC command in a case where the actual stress intensity factor is known, and to study the properties of the six and eight node elements when the $1 / \sqrt{r}$ singularity is simulated using the quarter point node location for the mid-side nodes. The far-field stress is a uniform stress applied at the top of the two-dimensional plate. Five meshes with differing levels of refinement, shown in Figure 14 were analyzed. In each mesh, symmetry was used to permit only one quarter of the plate to be modeled. Comparison of the stress field at the edge of the crack for the five different meshes is shown in Figure 15.

All five models were constructed starting with the regular rectangular mesh shown in Figure 14(a). Following an initial analysis with the rectangular mesh, each subsequent model was refined to study the rate of convergence of the mesh. The first refinement replaced the two rectangular elements at the crack tip with four triangular elements, as shown in Figure 14(b). To improve the capability of the model to predict the stress intensity factor, the $1 / \sqrt{r}$ singularity was introduced by moving the mid-side nodes of the two triangular elements to the quarter points. Next, a level five refinement at the crack tip node was created by using the automatic mesh tool. Refinement occurred on the model with a triangle element at the crack tip. The resulting mesh is shown in 
Figure 14(c) with a zoomed view of the area immediately around the crack tip shown in Figure $14(\mathrm{~d})$. The main purpose of this refinement was to investigate the capability of predicting the stress field near the crack without a singularity element. Finally, the midside nodes in the elements adjacent to the crack tip were moved to the quarter points to create the singularity once again. Figures 15 and 16 show the $\sigma_{y y}$ stress acting on the horizontal plane, extending from the crack tip to the edge of the plate. It is apparent from the model that all of the models converge to reasonably consistent values roughly a half inch away from the crack tip, which is half the length of the crack.

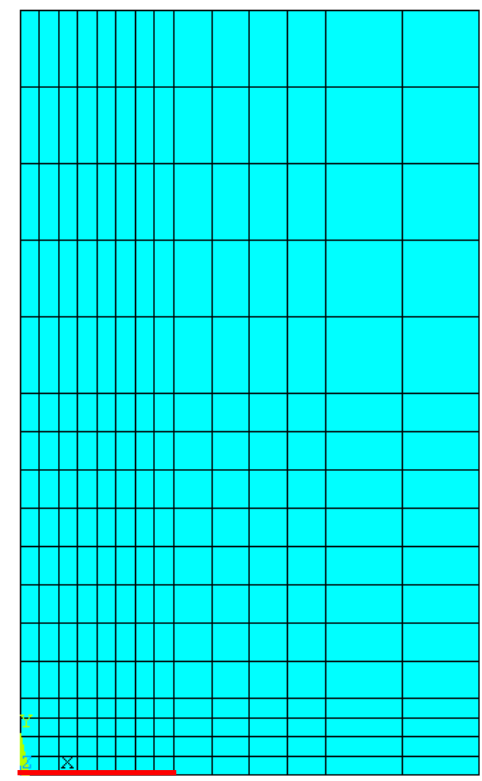

(a)

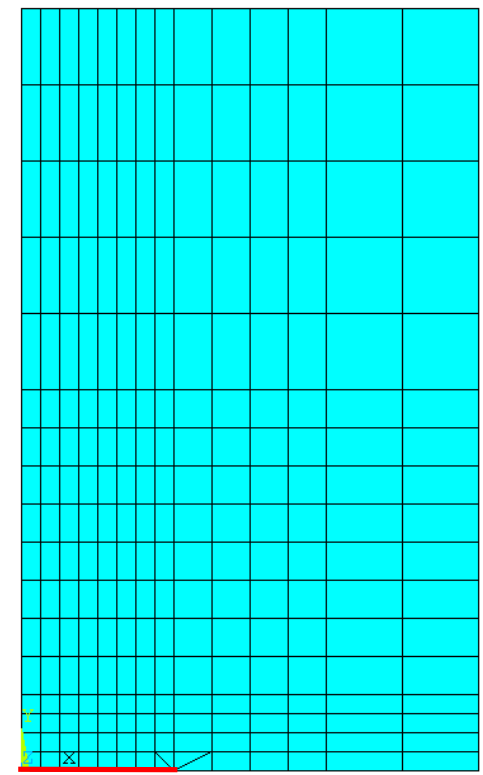

(b)

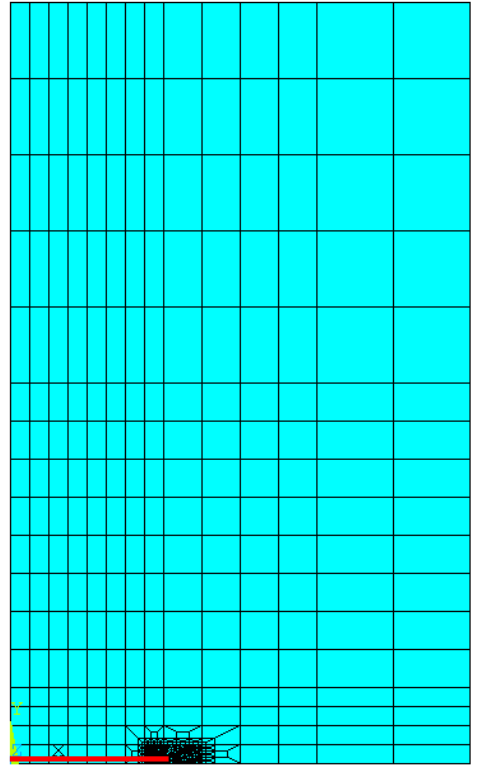

(c)

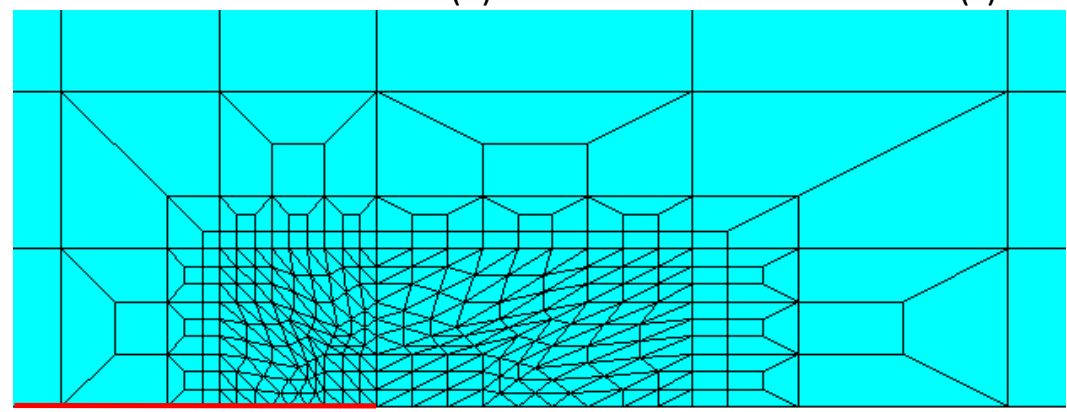

(d)

Figure 14: Plate Meshing, crack displayed in red 


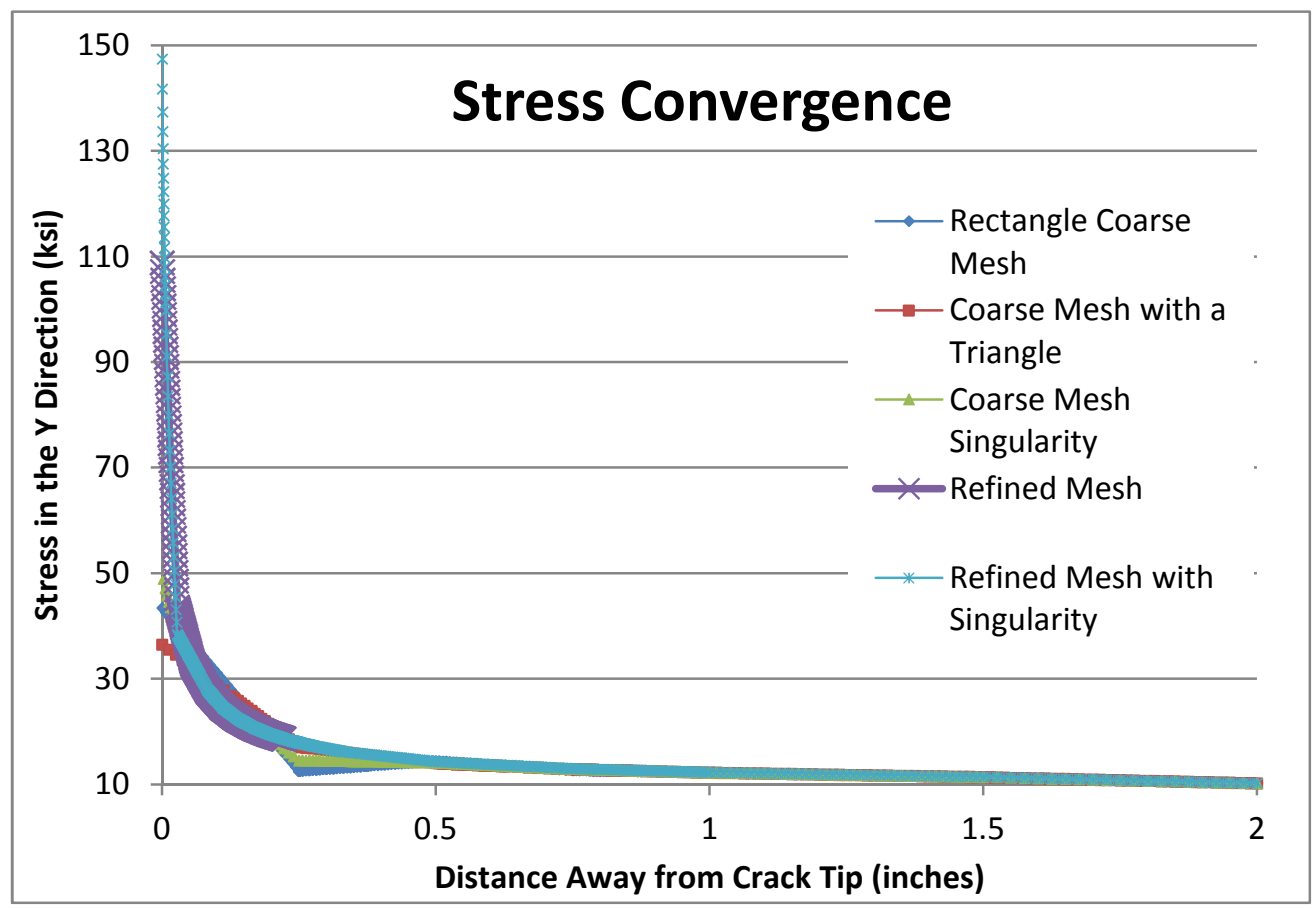

Figure 15: Stress along the Crack

In Figure 15, the region within the half inch closest to the crack is shown to permit the differences between the solutions to be calculated. None of the models predict infinite stresses at the crack tip, because the stresses in the singularity elements are not actually evaluated exactly at the singular node. However, it is seen that adding singularity elements does lead to larger predicted stresses than comparable meshes without the singularity elements. Obviously, it would be impossible for the software to provide output stresses exactly reflecting the singularity. 


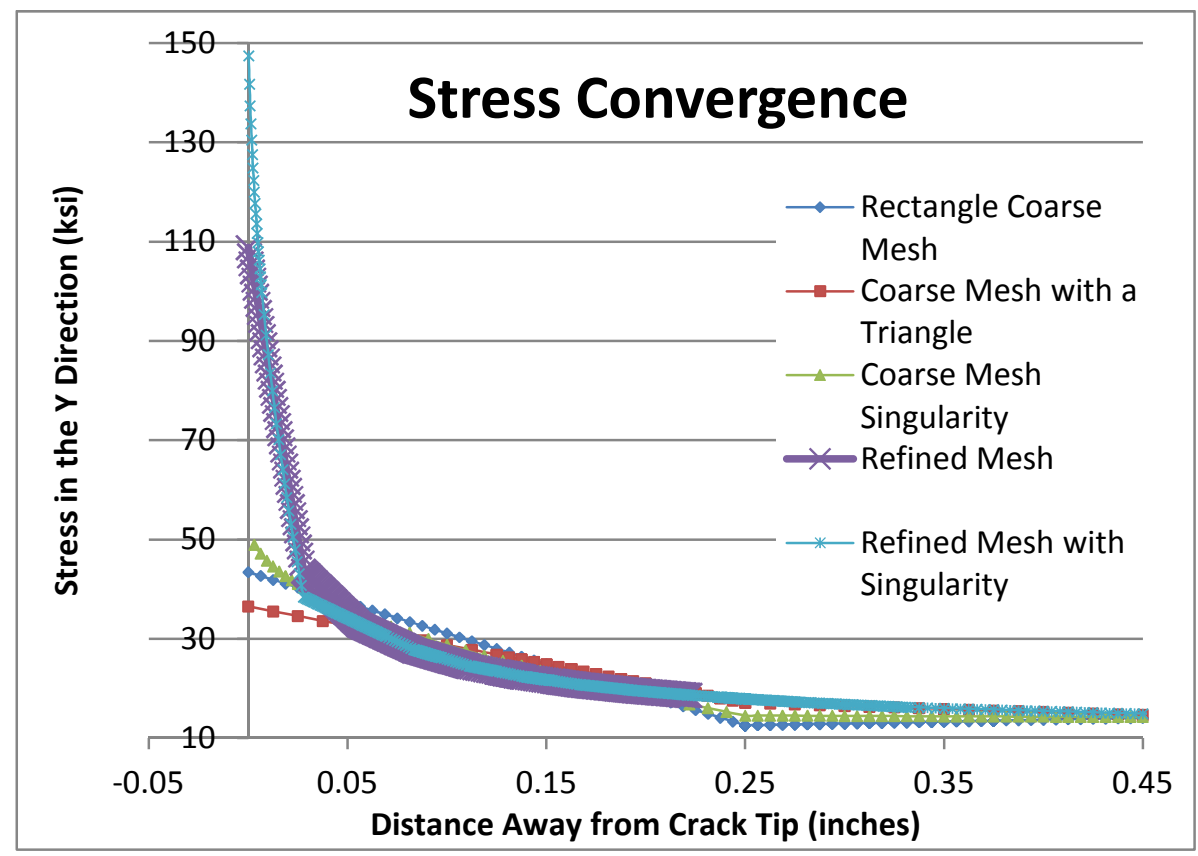

Figure 16: Stress Closest to the Crack

Large stresses were expected at the crack tip with an exponential decrease to the far-field stresses as the distance from the crack tip increases. The two refined models showed this behavior except right at the crack tip, where the analytical solution would predict a stress singularity, which cannot be reproduced exactly in a numerical model. The coarse meshed models were much less effective. The level five refinement using singularity elements was the closest to the expected results. It is believed that the singularity exists in this model, but stresses at the singularity cannot be reported. The refined models with and without the singularity elements were used to verify the stress intensity factor using the KCALC command, which uses a displacement curve fit for the region adjacent to the crack to estimate the stress intensity factor according to the ANSYS Theory Manual. Each model was executed for a half-crack with symmetrical boundary conditions for plane stress. Koiter indicated that the correct multiplier for a one 
inch long half crack in a plate with a half width of three inches is $F(a, b)=1.011$ (Tada, Paris, Irwin, 2000). The stress intensity relationship $K_{I}=F(a / b) \sigma_{y y} \sqrt{\pi a}$ with $F(a, b)=1.011$ and a far field stress $\sigma_{y y}=10 \mathrm{ksi}$, yields a stress intensity factor of $K_{I}=17.9203 \mathrm{ksi} \sqrt{\mathrm{in}}$.

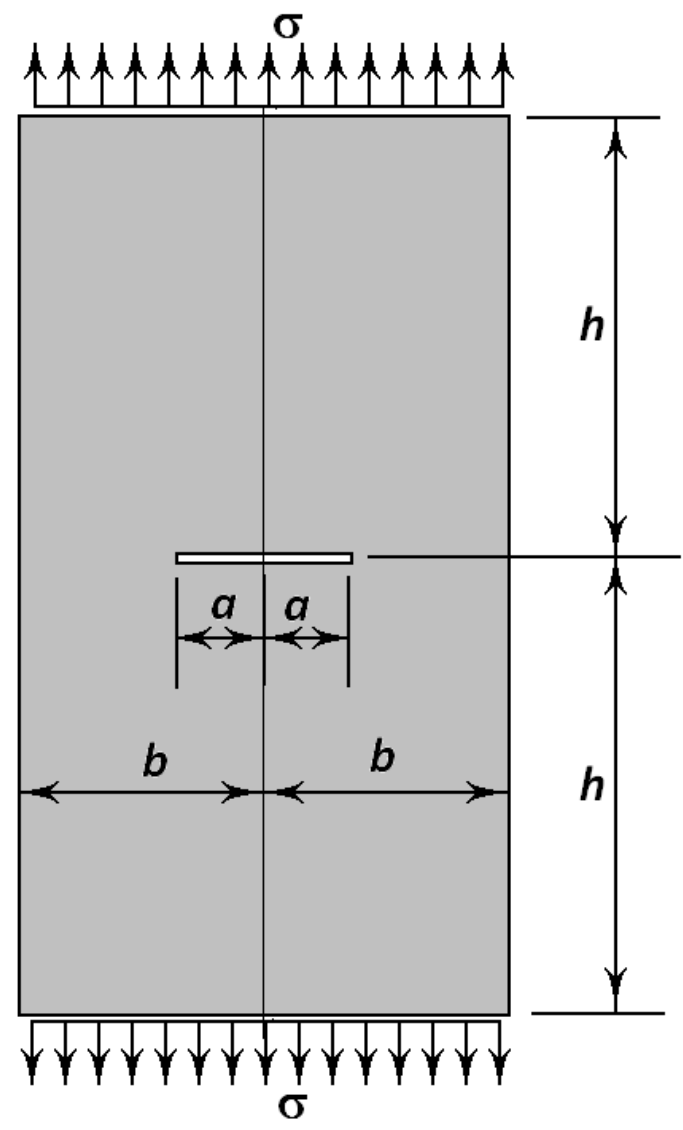

Figure 17: Finite Center Crack (Tada et al, 2000)

The stress intensity factor was evaluated for all five models, to investigate both the effectiveness of mesh refinement in the absence of the singularity elements, and the effectiveness of the singularity elements. Both plane strain and plane stress models were constructed, with the plane stress models providing somewhat improved results. The predicted stress intensity factors are given in Table 1. 
Table 1: Stress Intensity Factor Predictions, Finite Plate with Center Crack

\begin{tabular}{|l|l|c|}
\hline Mesh & $\begin{array}{l}\text { Predicted Stress Intensity } \\
\text { Factor } K_{I}\end{array}$ & Percent error \\
\hline $\begin{array}{l}\text { Coarse Rectangular mesh, no } \\
\text { singularity }\end{array}$ & $5.452 \mathrm{ksi} \sqrt{\text { in }}$ & $-69.6 \%$ \\
\hline $\begin{array}{l}\text { Coarse mesh with triangular } \\
\text { elements, no singularity }\end{array}$ & $14.445 \mathrm{ksi} \sqrt{\text { in }}$ & $-19.4 \%$ \\
\hline Refined mesh, no singularity & $14.419 \mathrm{ksi} \sqrt{\text { in }}$ & $-19.5 \%$ \\
\hline $\begin{array}{l}\text { Coarse mesh with singular } \\
\text { triangular crack tip elements }\end{array}$ & $18.278 \mathrm{ksi} \sqrt{\text { in }}$ & $2.0 \%$ \\
\hline $\begin{array}{l}\text { Refined mesh with singular } \\
\text { triangular crack tip elements }\end{array}$ & $18.180 \mathrm{ksi} \sqrt{\text { in }}$ & $1.4 \%$ \\
\hline
\end{tabular}

The tabulated values indicate several valuable features for fracture modeling. The first observation, from the two coarse meshes without singularity is that the triangular elements at the crack tip outperform the rectangular elements. Secondly, by comparing the coarse and refined meshes with triangular elements but no singularity elements indicates that simply refining the mesh does not significantly improve the $K_{I}$ calculation. This behavior should be expected, since the KCALC command only uses information from the element directly adjacent to the crack tip. The third observation is that introducing singularity elements at the crack tip provides significant improvement in the stress intensity factor calculation, even without accompanying mesh refinement, and that mesh refinement provides only small incremental improvement beyond that point. The stresses plotted in Figures 15 and 16, while indicative of the overall mesh refinement, do not provide any information about the accuracy of the stress intensity factor information, because the stresses are not used in the $K_{I}$ calculation.

The stress intensity calculation shows that ANSYS does, in fact, incorporate a singularity that can be exploited in two-dimensional models, by using the KCALC 
command, together with singularity elements. The situation in three dimensions is somewhat more complex. The KCALC command is not available in this case, so it is necessary to use some form of domain integral technique, such as a numerical $\mathrm{J}$ integral using the CINT command, together with three-dimensional singularity elements. Rather than pursue this approach, which is quite complicated to implement in ANSYS, given the necessity of introducing the singularity elements manually within complex threedimensional regions, and the more complex stress field around any incipient cracks, it was decided to focus instead on investigating the likely sites of crack initiation by examining the detailed local geometry of various two- and three-dimensional models that approximate to some degree the actual connection of interest.

\subsubsection{2-D T-connection Models}

In order to gain additional insight into the types and locations of stress concentrations of interest at the web-connection plate intersection, it was decided to next model a simple two-dimensional version of the connection. This model is somewhat limited, in that it provides no information about the condition at the termination point of the weld, but it does allow some consideration of the relative stresses at the toe and root of the fillet welds. This model also allowed convergence of the stress field in the vicinity of the complex geometry, as the number of elements is increased to be studied. The model also allowed the relative convergence properties of four-node and eight-node quadrilaterals, or 3-node and 6-node triangles to be evaluated. For this purpose, a simple two-dimensional T-connection with fillet welds was modeled. The model was not intended to represent the web-stiffener connection, but simply to gain insight into the flow of stresses that might be expected at such a $\mathrm{T}$ connection under different constraint 
conditions. In the two-dimensional T-connections, a distributed tension force was applied along the top of the vertical plate, as shown in Figure 18. Four and eight node brick elements were used in these models. It should be noted that if singularities were to be included, only the six node triangle and the eight node quadrilateral would be available.

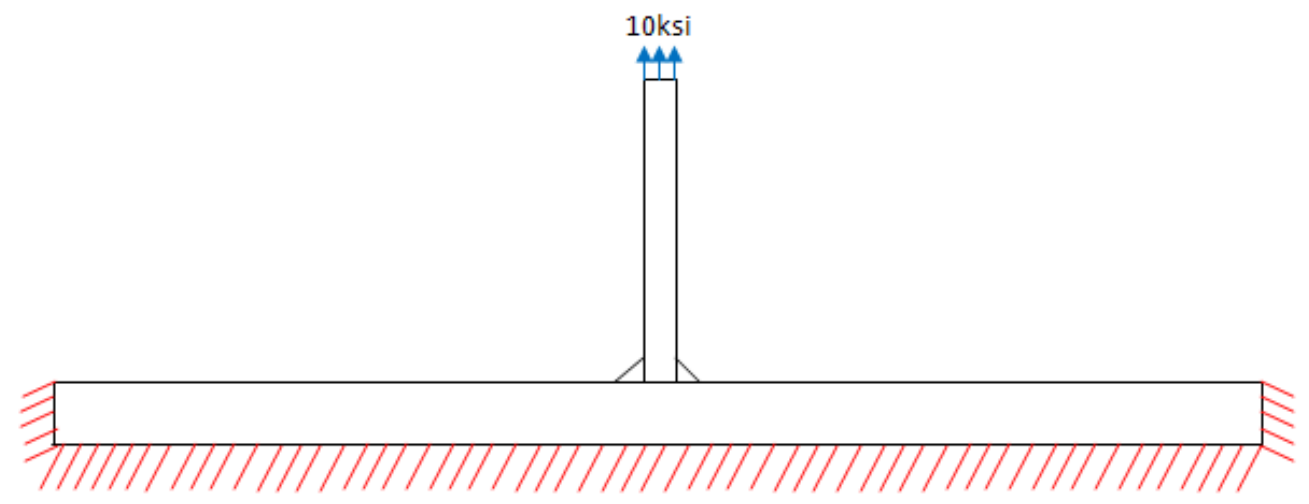

Figure 18: Boundary Condition

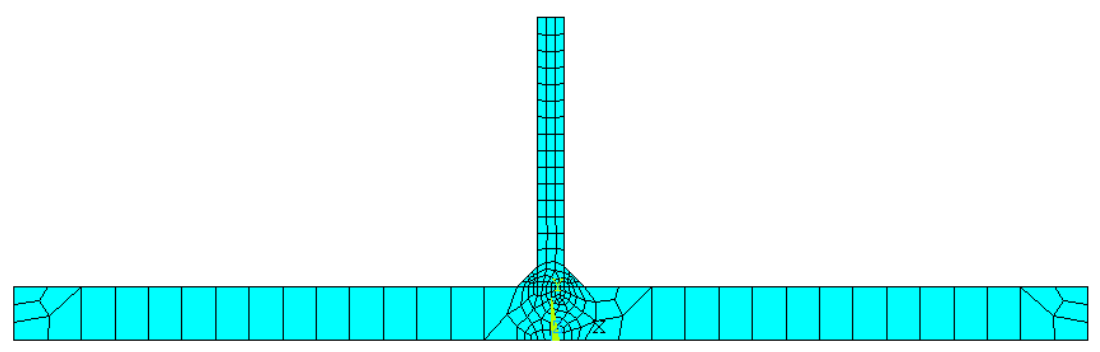

(a)

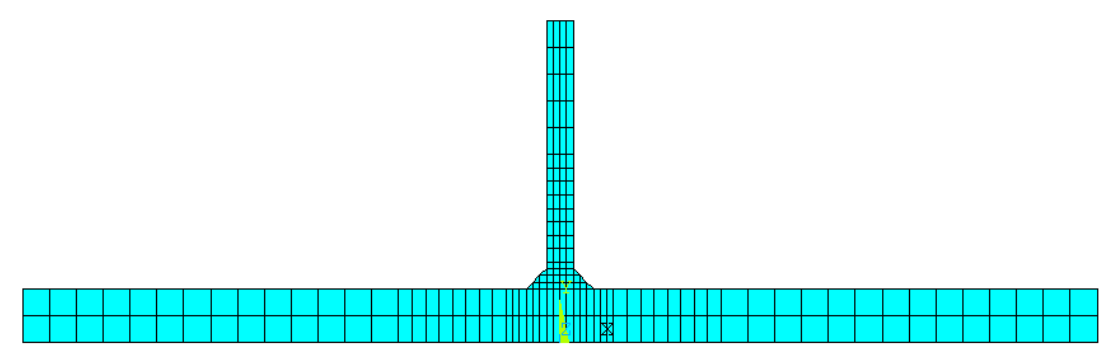

(b) 


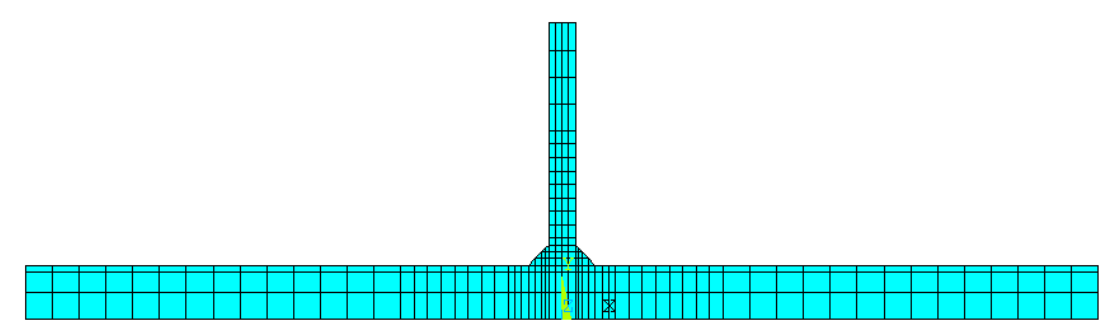

(c)

\section{Figure 19: Meshing for (a) 4 Node Element by Key Points, (b) 4 Node Element by Nodes, and (c) 8 Node Element by Nodes}

In order to investigate the potential for the root of the connection to act as a crack, the T-connection models included a small gap between the vertical and horizontal plates. As indicated in chapter 3 , no weld penetration into the vertical plate was assumed. in this model, and compressive residual stresses at the root of the welds were not considered. In the absence of a gap, which would occur under a full penetration weld, or if the vertical plate was compressed against the horizontal plate as a result of welding residual stresses, the highest stress concentration occurred at the toes of the fillet welds. Once the gap was added, the hot spot location moved to the root of fillet weld rather than the toe. Treating the small gap as a crack tip, the stress at the crack tip was about $24.6 \mathrm{ksi}$ and $23.5 \mathrm{ksi}$ with models constructed using the key point method and node method, respectively, using a four node element. The model constructed using key points and areas predicted slightly higher stresses because there are slightly smaller elements at the root of the fillet weld. Using the quadratic eight node elements resulted in a stress of $25.8 \mathrm{ksi}$, which is about the same stress, but this model had a significantly coarser mesh, verifying that the quadratic 8- node elements are providing comparable accuracy to the bilinear four-node element with a coarser mesh. 


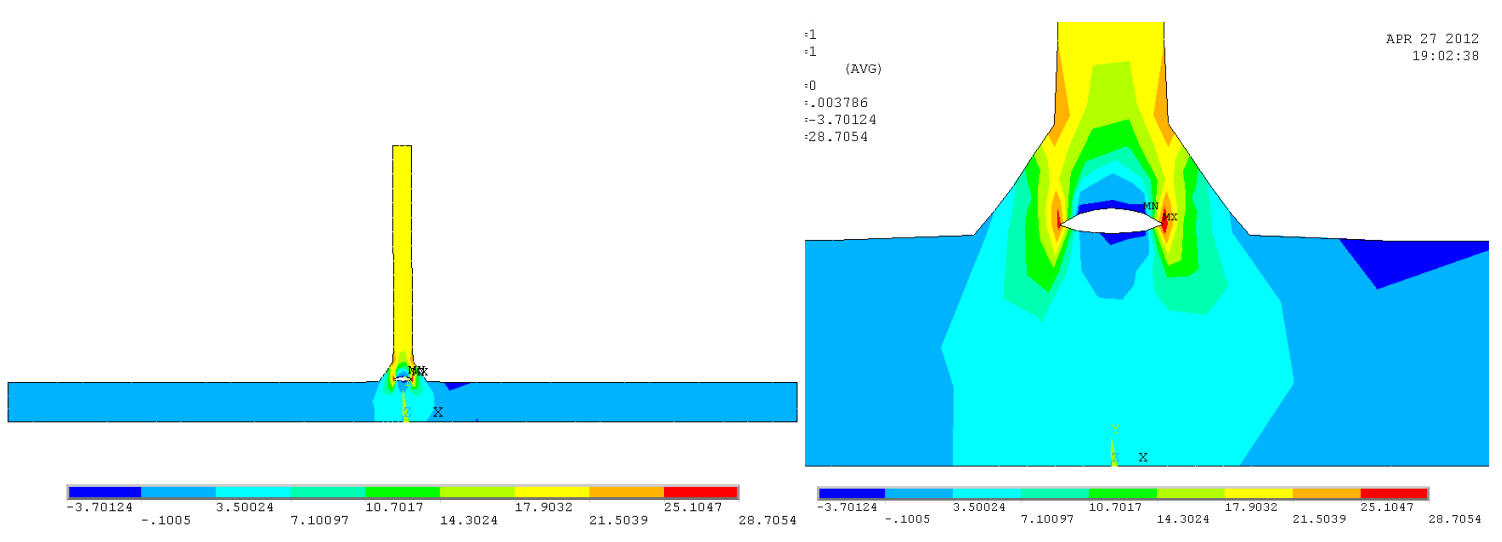

Figure 20: 4 Node Element Results By Key Points

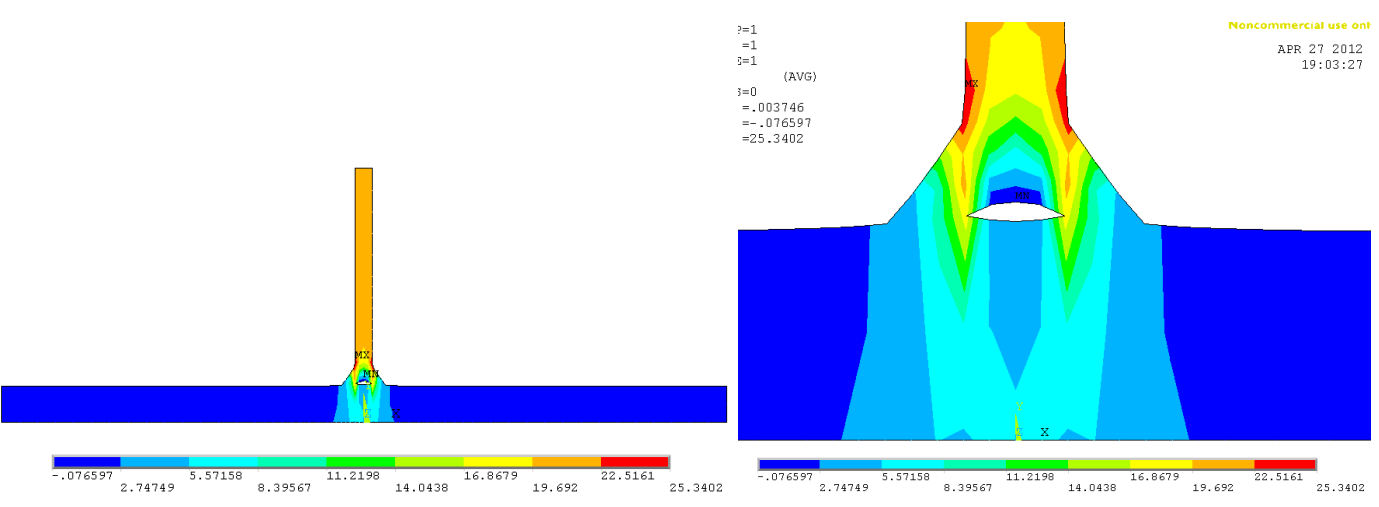

Figure 21: 4 Node Element created by Nodes

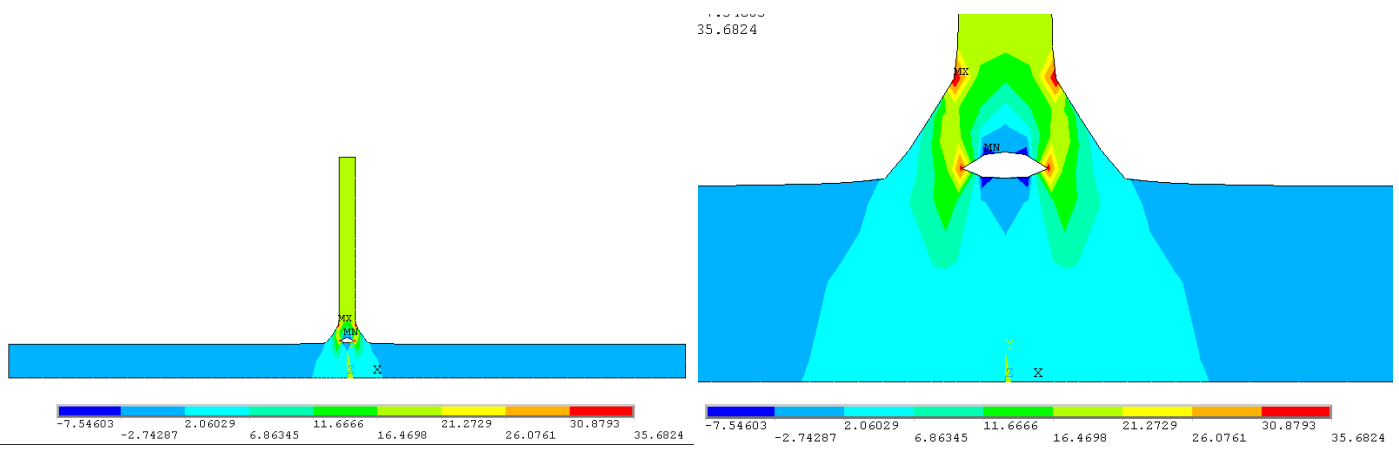

Figure 22: 8 Node Element

Several authors, (Chang \& Lee, 2009; Teng, et al, 2001) have indicated that the crack will start at the toe, and not the root, of the fillet weld. In Teng et al (2001), a mechanism is discussed wherein the weld causes the bottom plate to distort upward, 
introducing a compression region where the two plates intersect. In this case, no singularity would exist at the root of the weld, and a crack would not grow from the root of the fillet weld. Ferreira and Branco, (1991) provided correction factors for the stress intensity factor for cracks starting at the toe of the weld going through the plate thickness. The likely reasoning for choosing this site for the crack initiation is the presence of tensile residual stresses. These correction factors also incorporated the type of stress field causing these cracks. It was then an objective to model this and try to replicate high tension stresses at the weld toes resulting from applied loadings.

The previous study included an initial crack at the fillet weld root, assuming that the root is a likely source of cracking. Following the above considerations, it was decided to investigate the stress state that would exist if either the welds are full penetration or the fillet weld residual stresses cause the root to be in compression. To investigate this case, the model was modified to eliminate the crack between the two plates. For the purpose of the current studies, there was no attempt to reproduce the tensile residual stresses at the toes that have been reported in the literature. The first attempt to produce maximum stresses at the weld toes connected the elements forming the two plates as if they were welded. The forces were upward at the end of the vertical plate and only the top corners of the horizontal plate were pinned, as shown in Figure 23. The outcome, shown in Figure 25, did show an angle distortion as in the paper. The toes on the horizontal plate developed a stress of $73.65 \mathrm{ksi}$ in tension. 


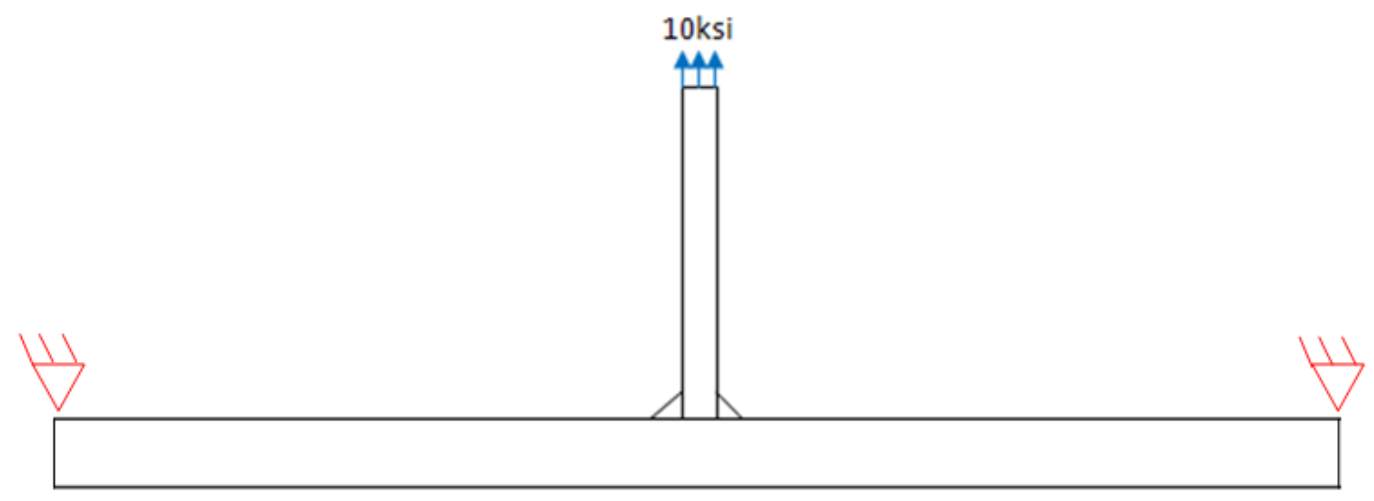

Figure 23: 2-D Boundary Condition Pin
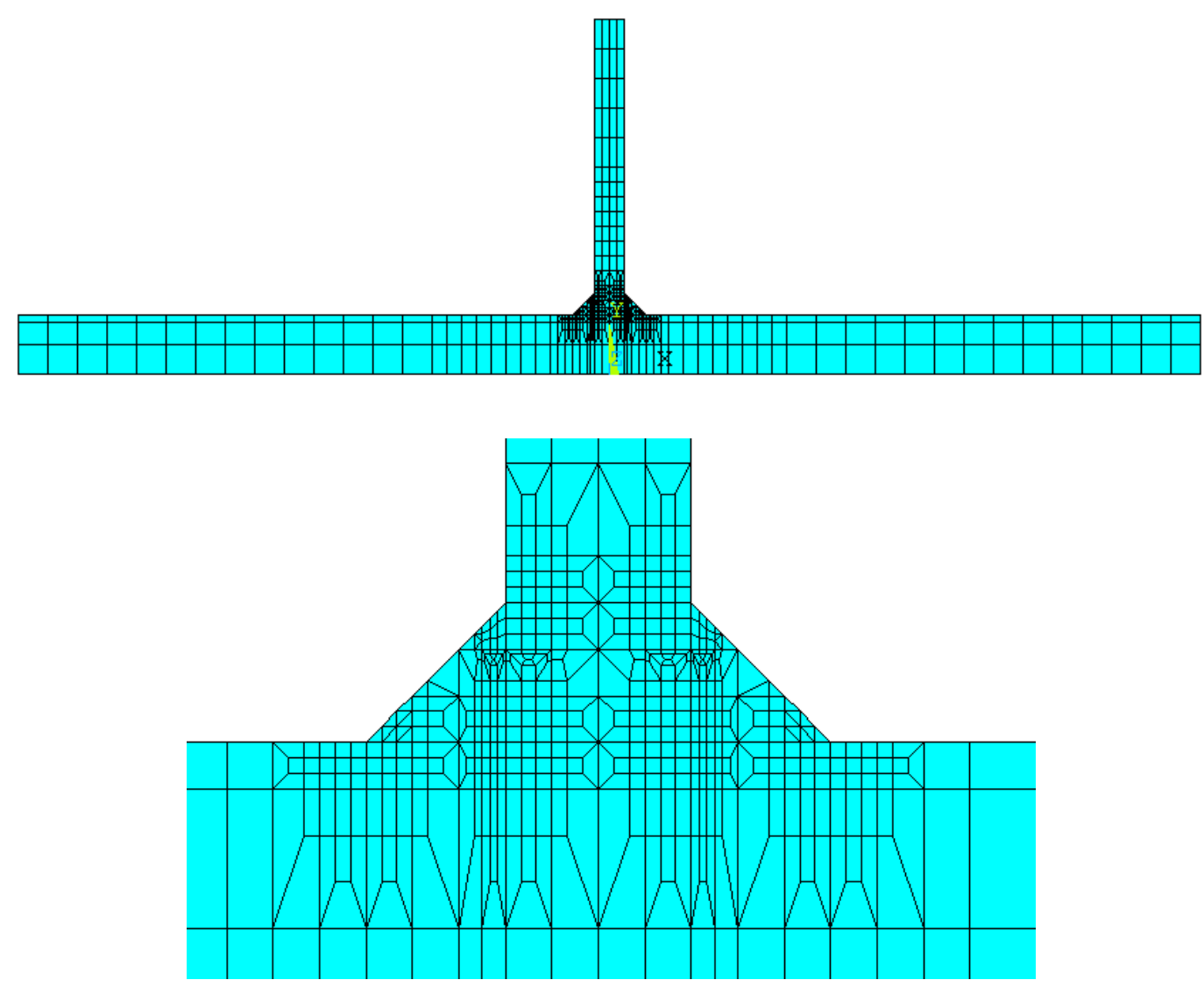

Figure 24: Meshing for all 2-D Models 

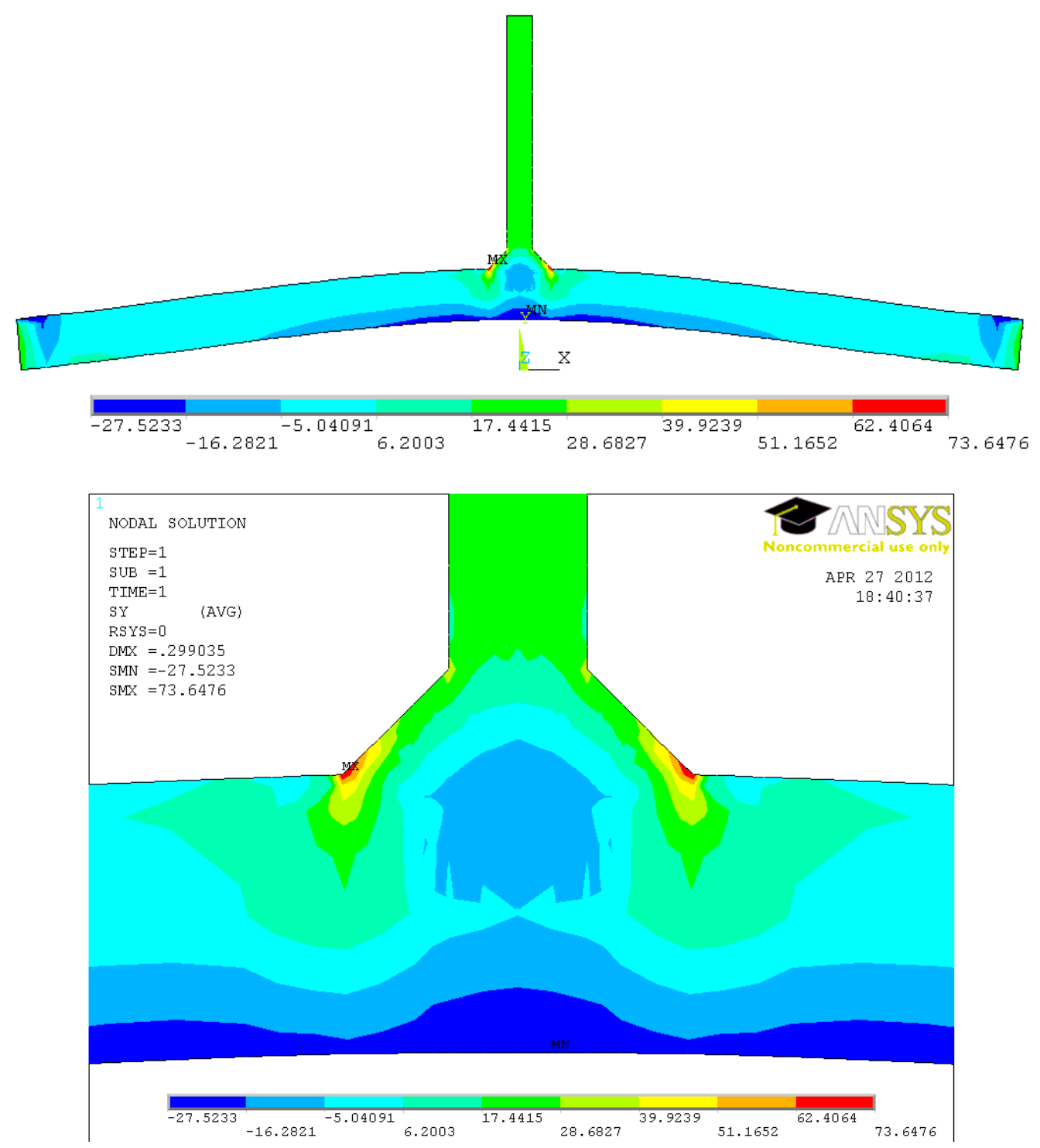

Figure 25: 2-D T-connection without Gap

Another attempt was made to create a large stress concentration in the toe adjacent to the vertical leg. In order to do this, the horizontal plate was restrained against vertical motion by applying restraints against vertical translation at the bottom of that plate, as shown in Figure 26. Under this highly constrained state, the maximum stress was located at the toe on the vertical plate as seen in Figure 26. It was about half of the previous stress. Since both sites of likely crack initiation predicted by Ferreira and 
Branco, (1991) were replicated, using the two limiting boundary condition cases, the toes may be considered as the likely sites of crack initiation.
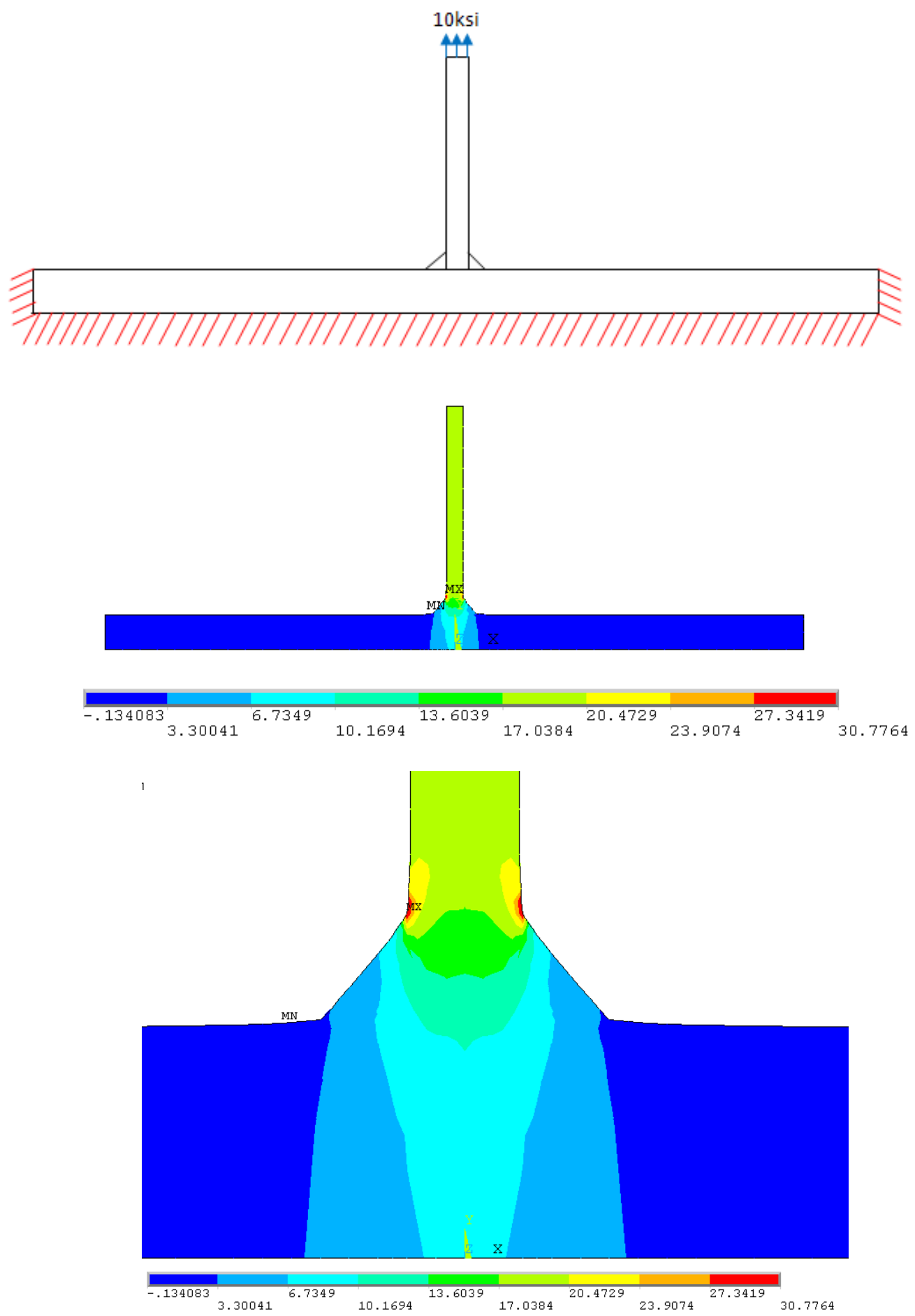

Figure 26: Fixed Boundary Conditions of 2-D T-connection 49 
The importance of the toe verses the root of the fillet weld depends on the boundary conditions, the presence of an initial crack at the root of the fillet welds, and the existence of compressive residual stresses at the root of the fillet welds.

A final study using the two dimensional T-connection model was completed to investigate the influence of different boundary conditions. Four boundary conditions were investigated. The toe stress, with the top corners of the horizontal plate pinned, is about the same both with and without a crack at the root of the welds. All the locations in question have relatively high stress concentration factors: the toes adjacent to the horizontal plate have a factor of 10 . This factor was calculated by comparing it to the applied tensile stress. In Figure 28, the stress in the toe of the weld is almost twice that of the root, and the root is in compression. This indicates that the toe is the likely site for a crack to start even without considering residual stresses. In Figure 29, rollers are added to the exterior ends of the bottom plate, resulting in fixity of the ends against rotation. The toes at the horizontal plate have a stress concentration factor of 20.2, while the root has a stress concentration factor of 16.5 , but the stress is compressive. Again, the weld toe is the likely place for the crack to start. 

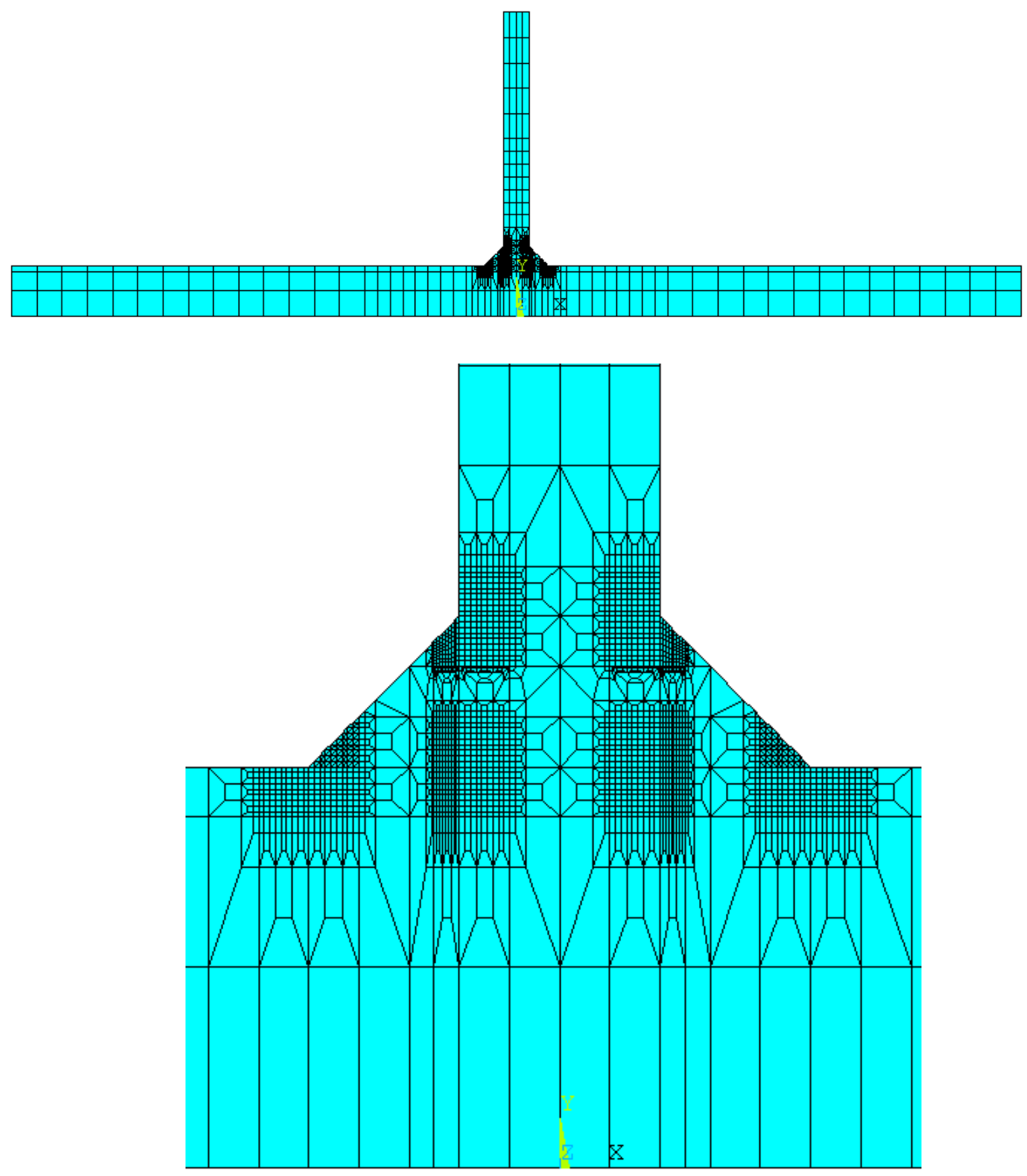

Figure 27: Mesh for Four Boundary Conditions 

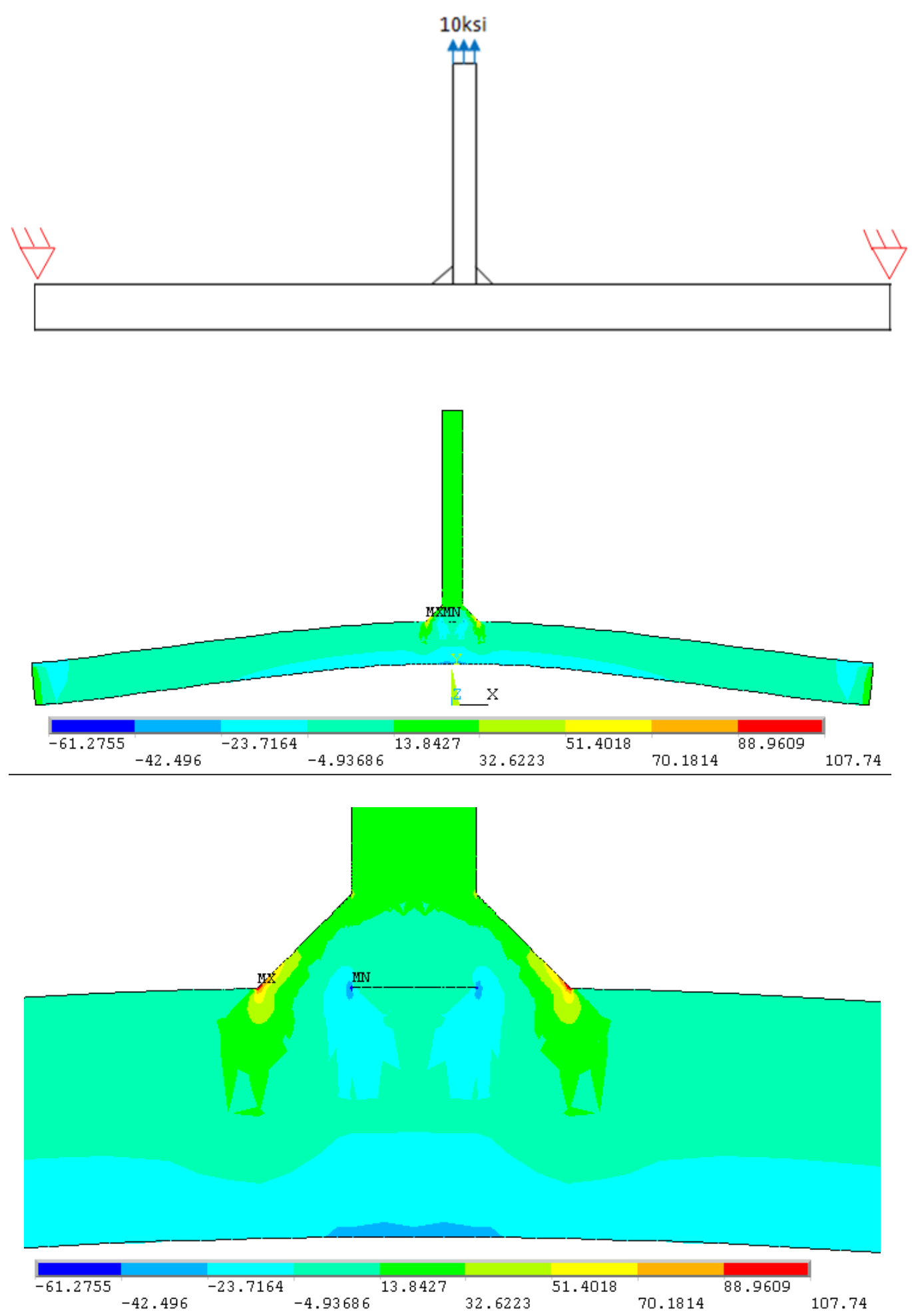

Figure 28: Pin at top corners of bottom plate 

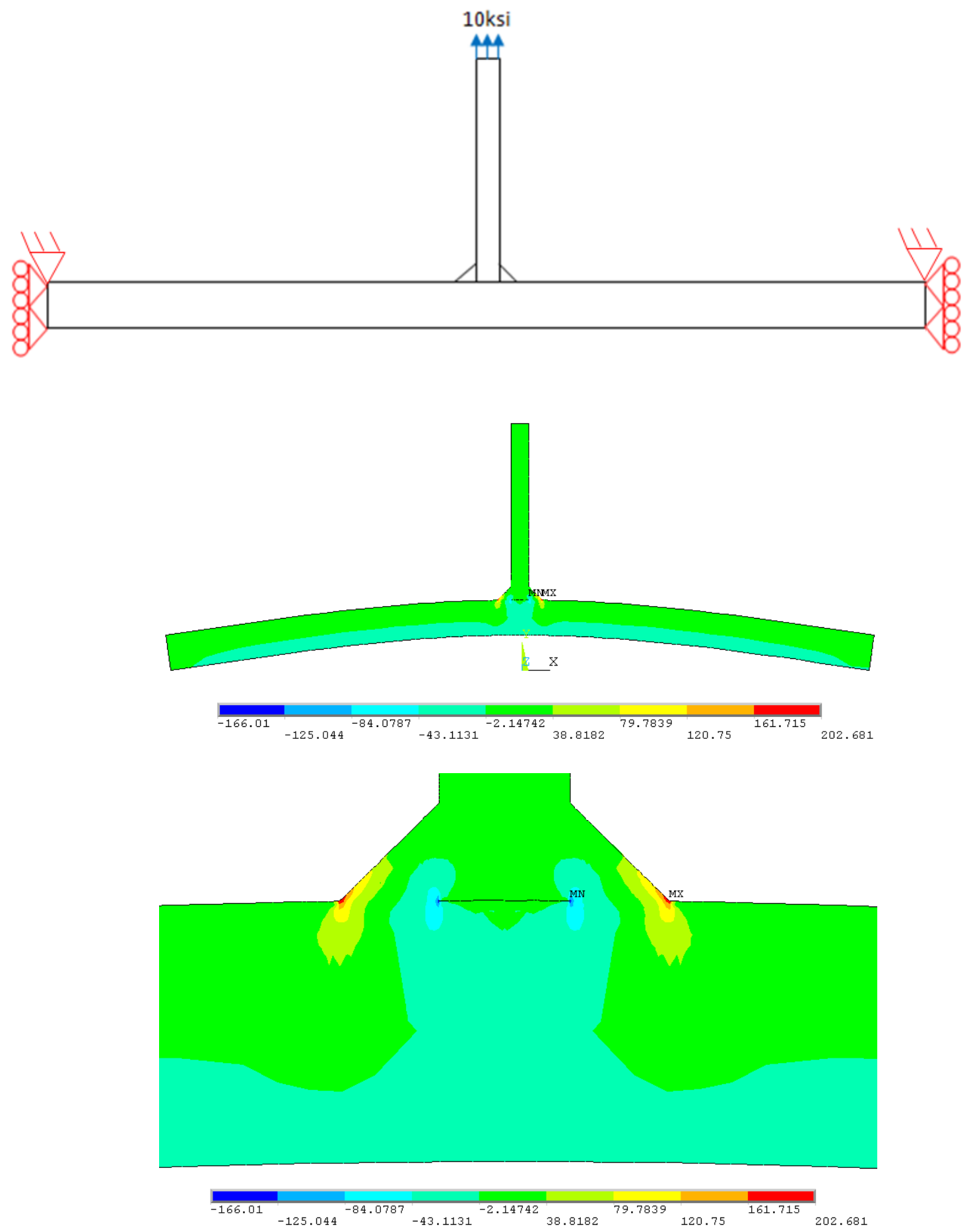

Figure 29: Pin at top corners of bottom plate and rollers along verticals 
The next boundary condition considered only increases the fixity slightly, by replacing the rollers on the vertical ends of the horizontal plate with complete fixity, as shown in Figure 30. The stress concentration factors are significantly smaller than the previous conditions: one toe has a factor of 9.7. The root is again in compression with these boundary conditions, the toe adjacent to the horizontal plate remains the most likely place for the crack to start.
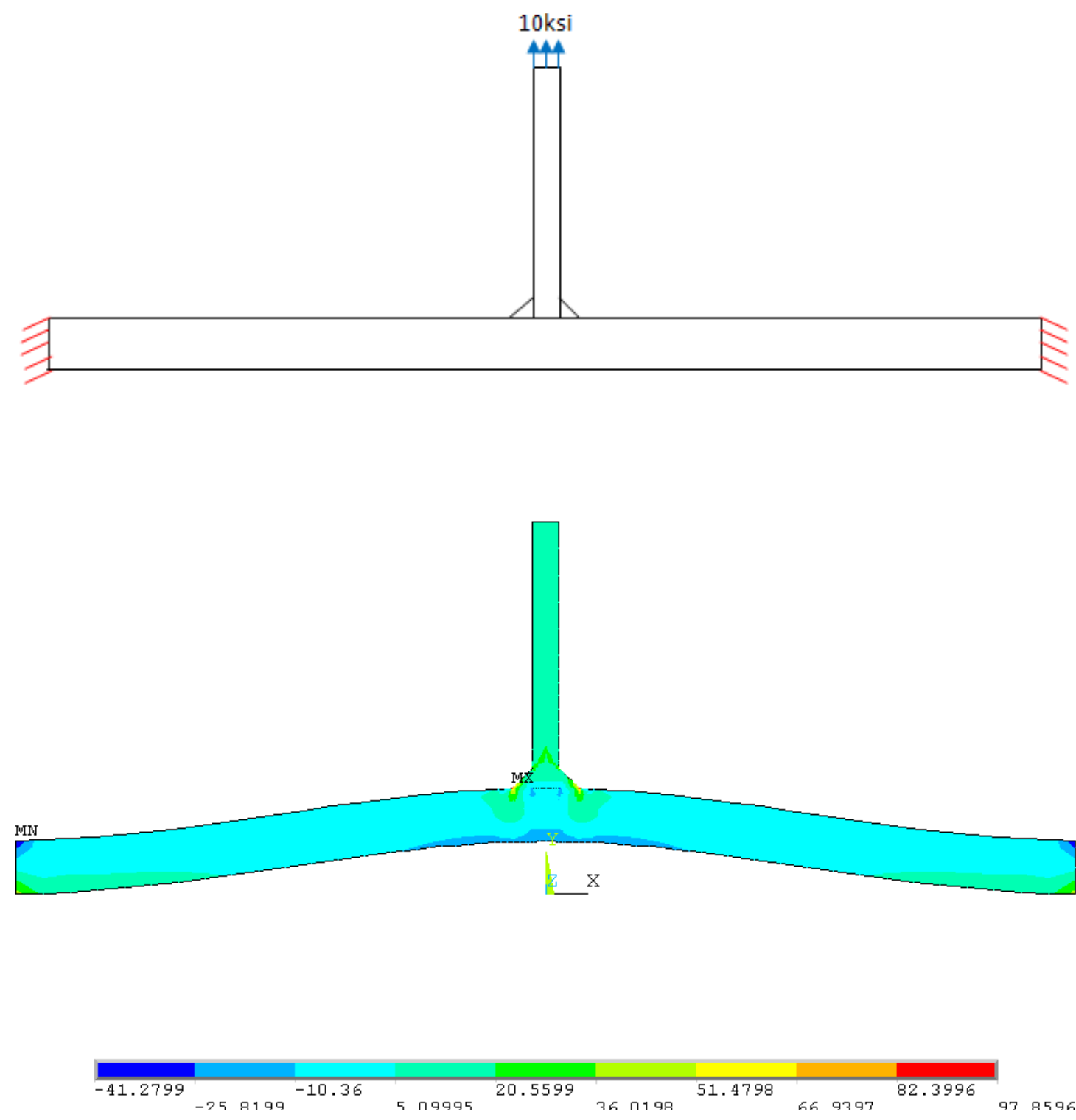


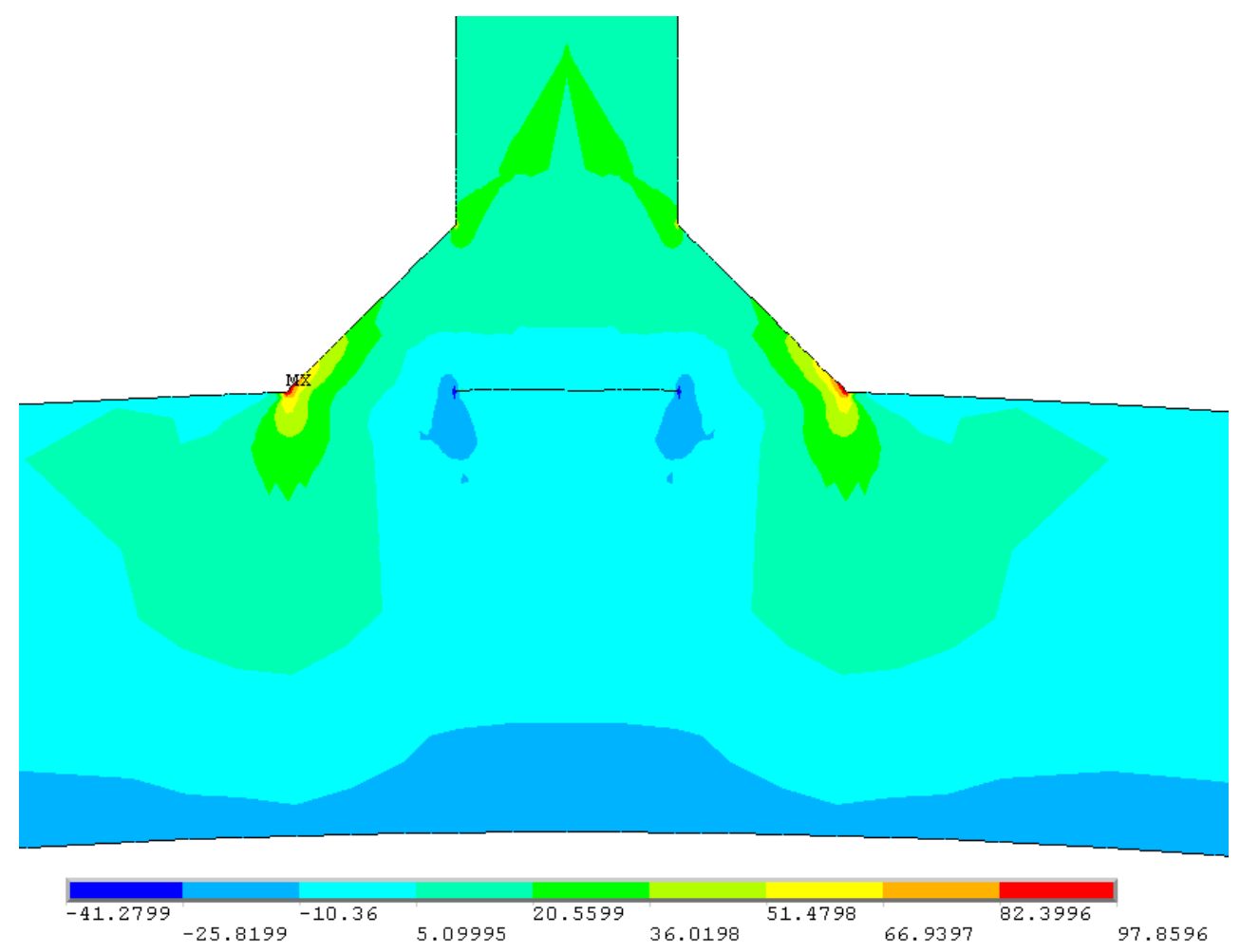

Figure 30: Fixed along verticals of the bottom plate

The exact degree of resistance of the horizontal plate to translation depends upon conditions out of plane; so to examine the most highly constrained case, the bottom of the horizontal plate was constrained against vertical translation, as shown in Figure 31. When the bottom of the plate is fixed as well, the stress at the root of the fillet weld is higher than that of the toe in Figure 26. The toe stress is about the same as if without a crack. This is the only boundary condition where the root is a more likely place for the crack to start. This is logical, because with this constraint state the stress flow simply moves around the hole to reach the fixed boundary on the bottom of the horizontal plate, rather than flowing laterally toward the ends of the horizontal plate. 

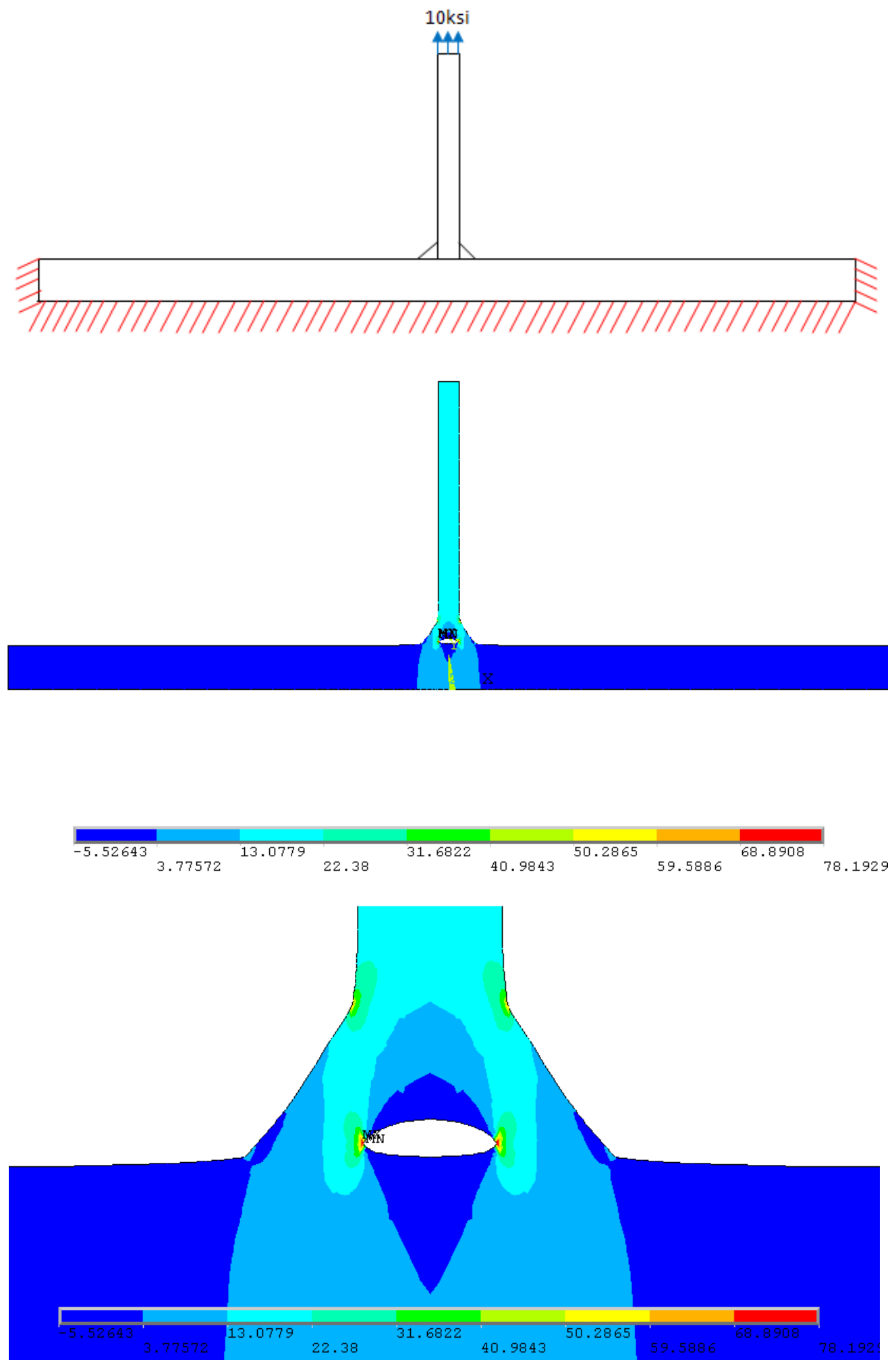

Figure 31: Fixed along bottom plate verticals and along bottom 
In the Carter Glass Bridge, the web plate between the end of the connection plate and the flange will provide partial fixity against translation, so an idealized set of boundary conditions intermediate between the second and fourth boundary conditions appears to be a reasonable approximation. Since the locations of the highest stress concentrations differ between these two boundary conditions, it is not possible to state definitively where crack initiation will occur upon these models. It does appear more likely that with only partial restraint the expected area of interest will be at the toe of the fillet weld, particularly if residual stresses and partial or complete penetration of the weld are also taken into account. It is more likely for a defect at the toe of the fillet weld to lead to a crack than at the root. This was considered in the results of the model of the actual girder, but it was decided to retain the crack between the web and connection plates in the full three-dimensional model.

\subsubsection{3-D T-connection Hot Spots}

After gaining insight from the two-dimensional studies, a simplified threedimensional study was undertaken. The geometry of the simplified three-dimensional model is shown in Figure 13. The three-dimensional study applied a concentrated load at the ends of the vertical plate as shown in Figure 32, in order to locate the stress concentrations at the end of greatest interest. The first model was constructed using eight-node brick elements. In an attempt to partially replicate the weld termination point, and the web gap created by the connector plate cut-out, the horizontal plate was extended beyond the edge of the vertical plate. Fixed boundary conditions were applied on sides A, B, and C of Figure 32.The stresses were examined at the weld end adjacent to side B. Figures 34 and 35 show the results of this simulation. The largest stress concentration is 
at the root of the fillet weld, but some larger stresses do occur at the toe adjacent to the connector plate model. This reinforces the fact that a crack could occur at either location.

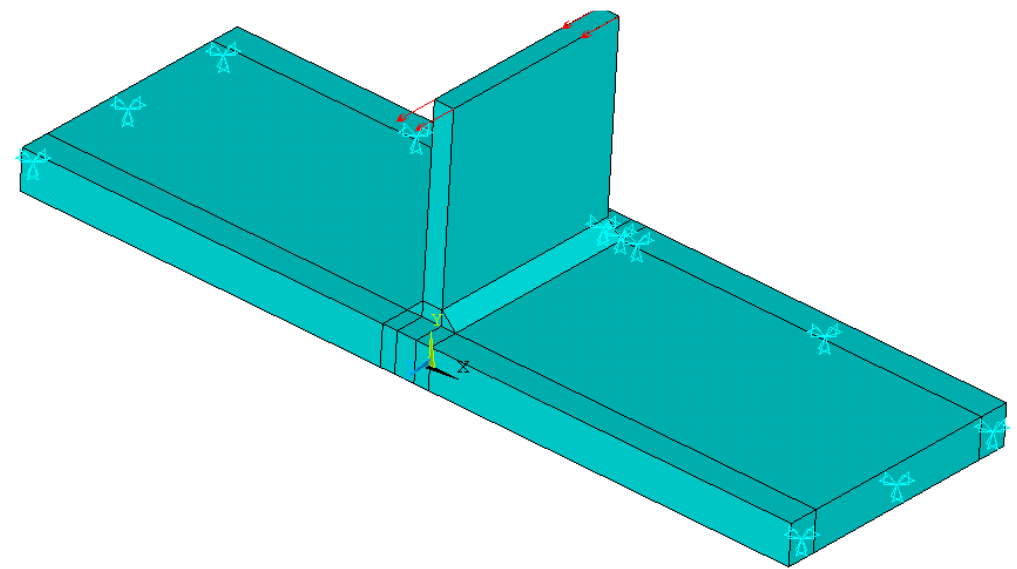

Figure 32: 3-D Boundary Conditions

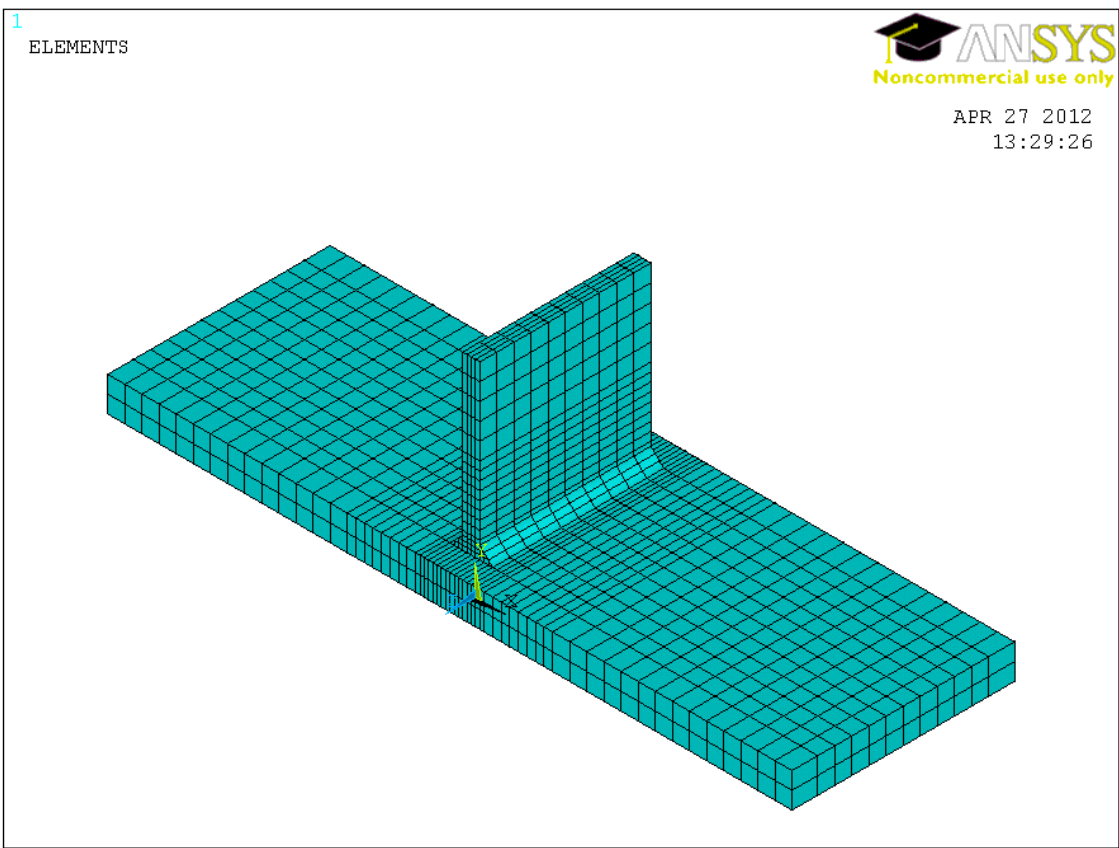

Figure 33: 3-D 8 Node Model Mesh 


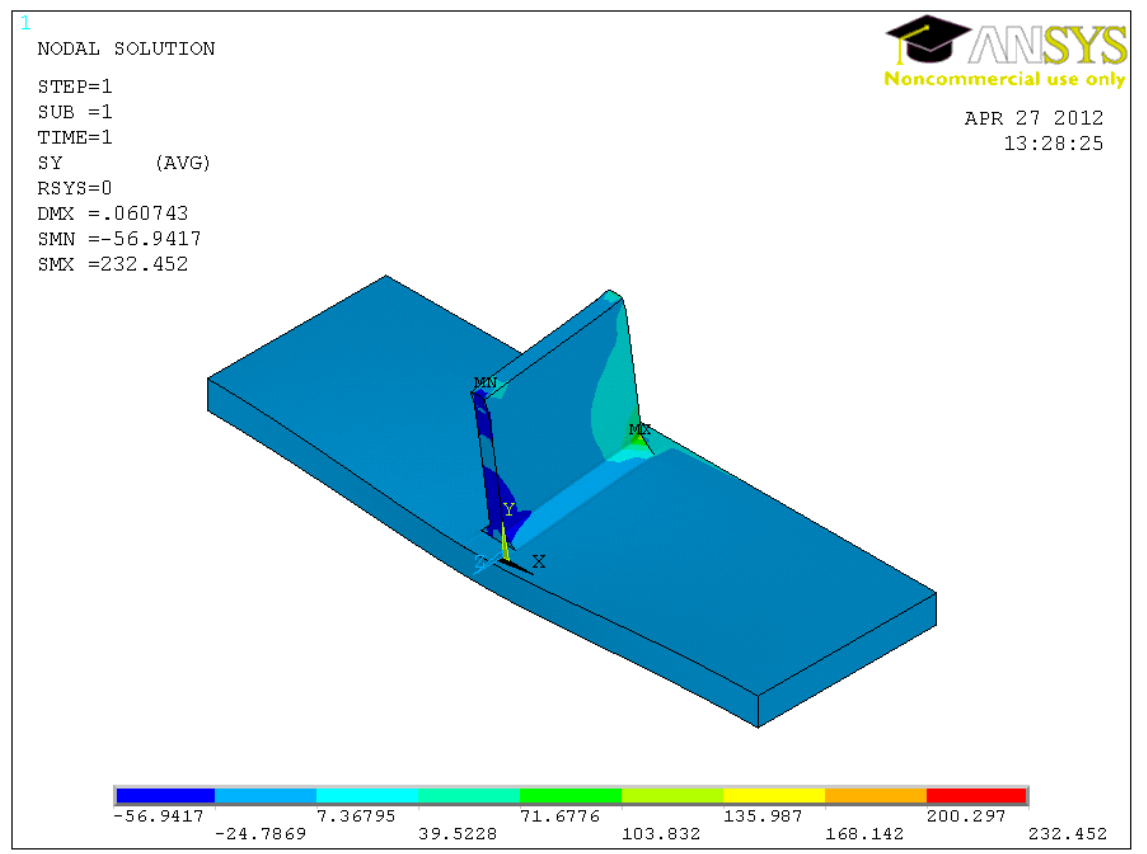

Figure 34: 3-D 8 Node Model Deflected Shape with Stress Contours

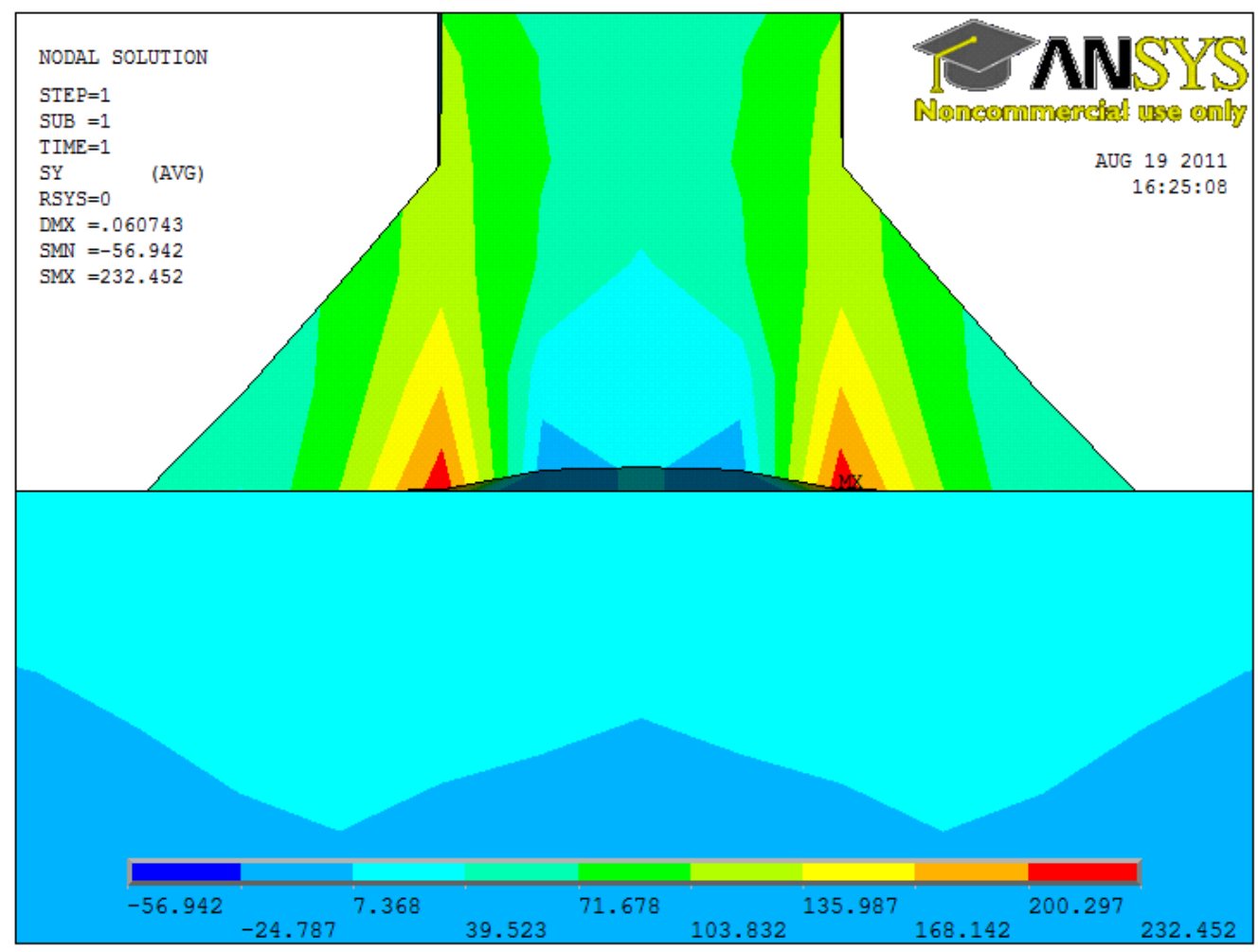

Figure 35: 3-D T-connection with 8 Node Element 
To improve upon the accuracy of the model, the eight node brick elements were then replaced with twenty node brick elements as shown in Figure 36, and the study was repeated. Figures 37 and 38 show high stresses at the top of the fillet weld, which was unexpected, since this had not appeared with the eight node elements. The stresses at the crack tip, provided by the root, increased and the stresses at the toe decreased when the mesh was further refined. The relatively high stress concentration, at the toe of the fillet weld reinforces the idea that the cracks might start at weld toes. The absence of a significant stress concentration at the toe adjacent to the "web plate" was somewhat surprising, and suggests that the restraint provided by the flange against web translation might be substantial.

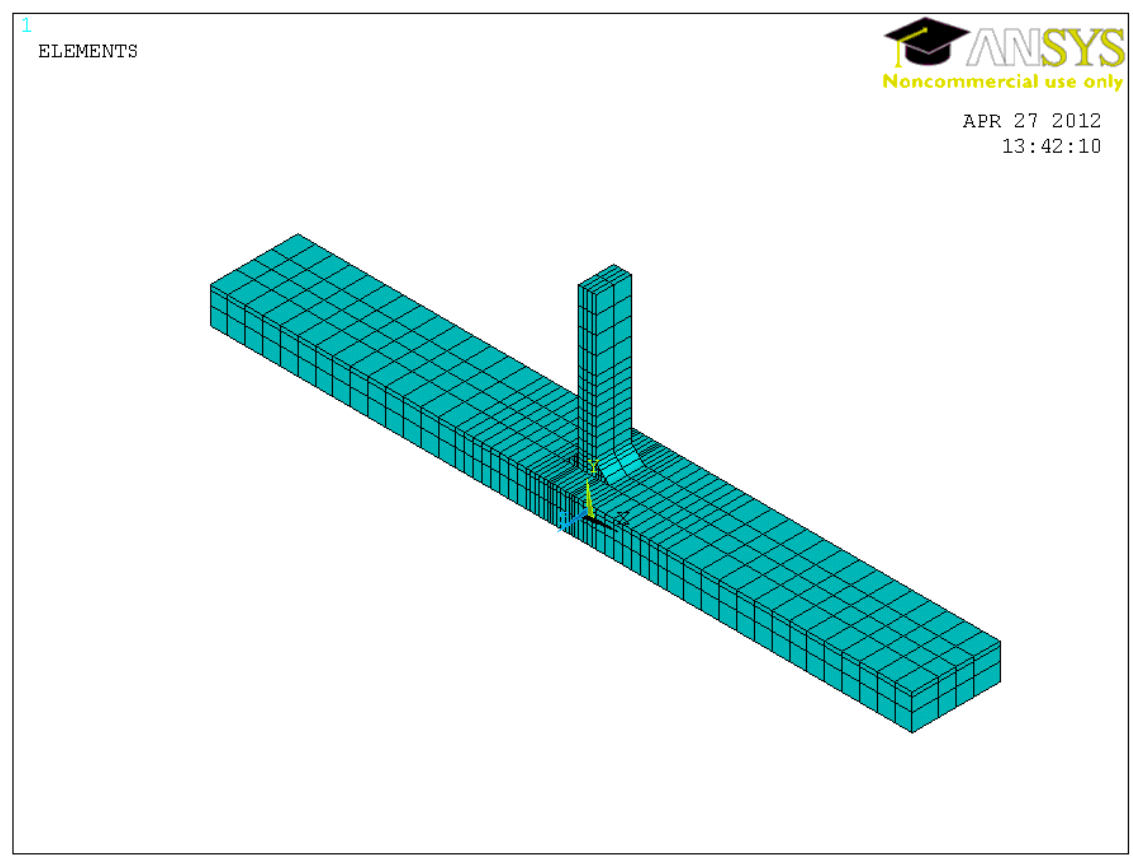

Figure 36: 3-D 20 Node Model Mesh 


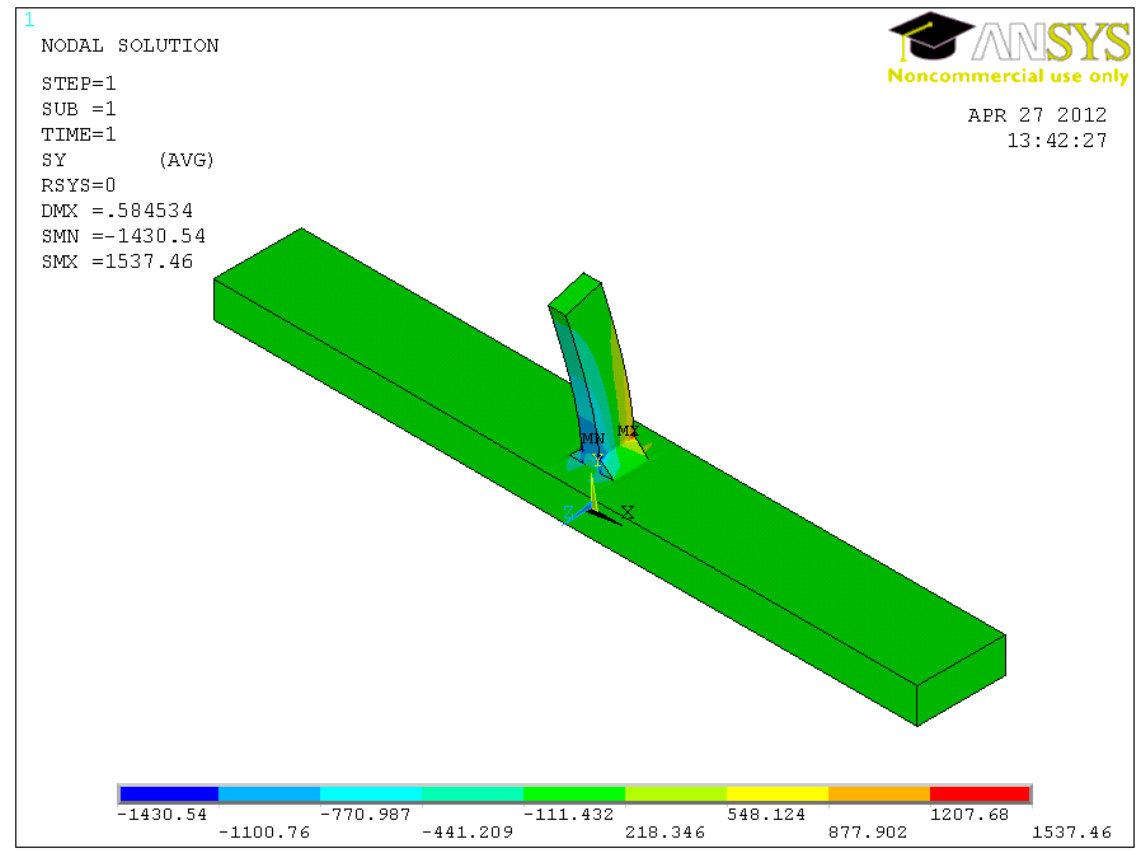

Figure 37: 20 Node Model Deflected Shape with Contours

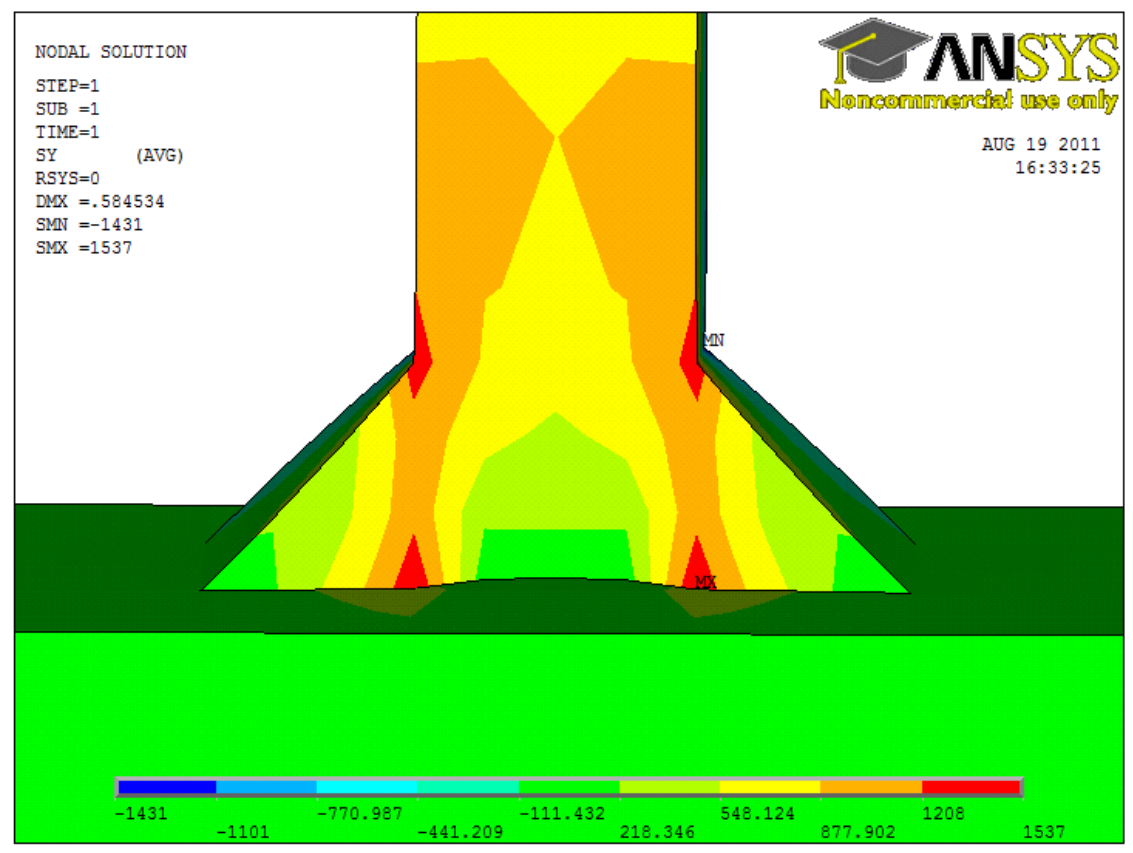

Figure 38: 3-D T-connection created with 20 Node Element

A closer look was made to look into hot spots for the geometry of the connection plate cut-out. This time a horizontal plate was attached to a stiffener with a cut-out by 
fillet welds as shown in Figures 39 and 40. This is still a simplified model but closer to the actual connection. The stress distribution occurred as shown in Figure 41. The largest stress concentration occurred at the root, as originally expected. The toe stresses are still a third of that value. This means that both parts of the fillet weld must be refined to give accurate results. It also can be concluded that the fillet weld information is vital for correct outputs. The constraint introduced at edge $\mathrm{B}$, where the flange connection would be, has a significant influence on the stress flow, and the location of the highest stress concentration.

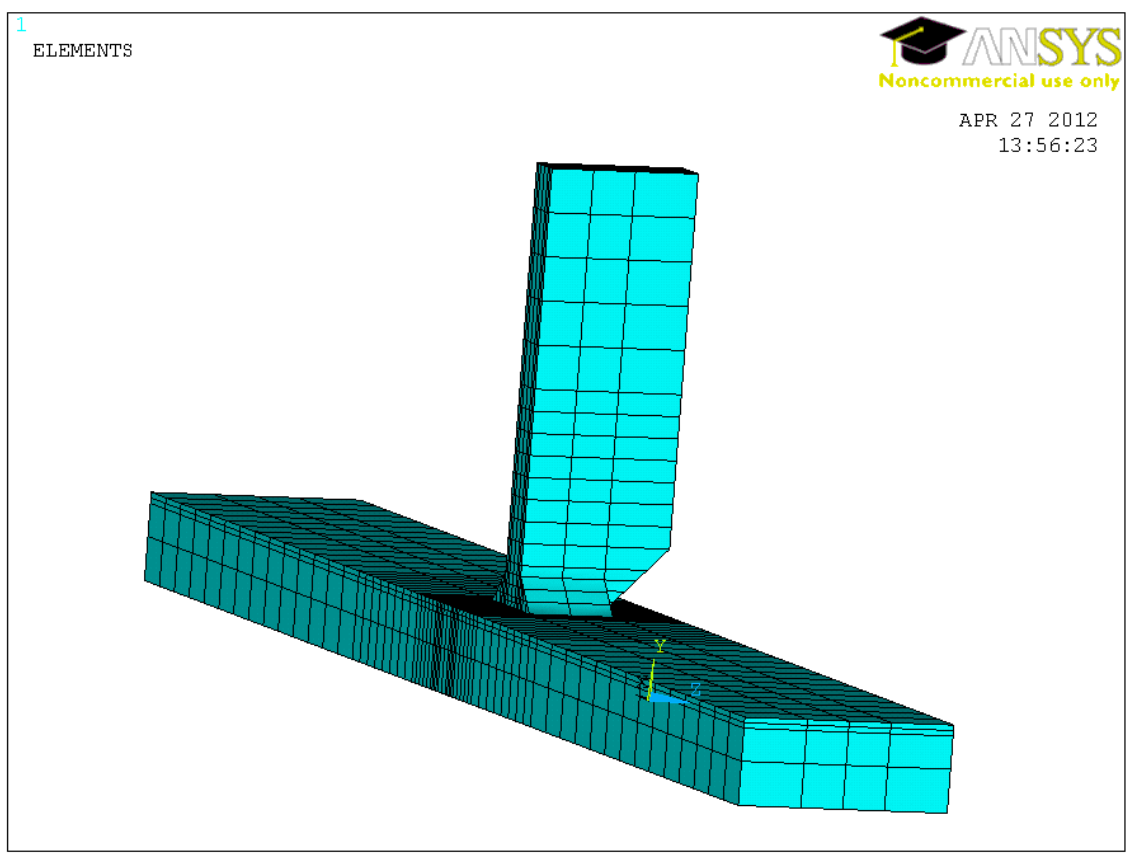

Figure 39: 3-D Cut-out Model Mesh 


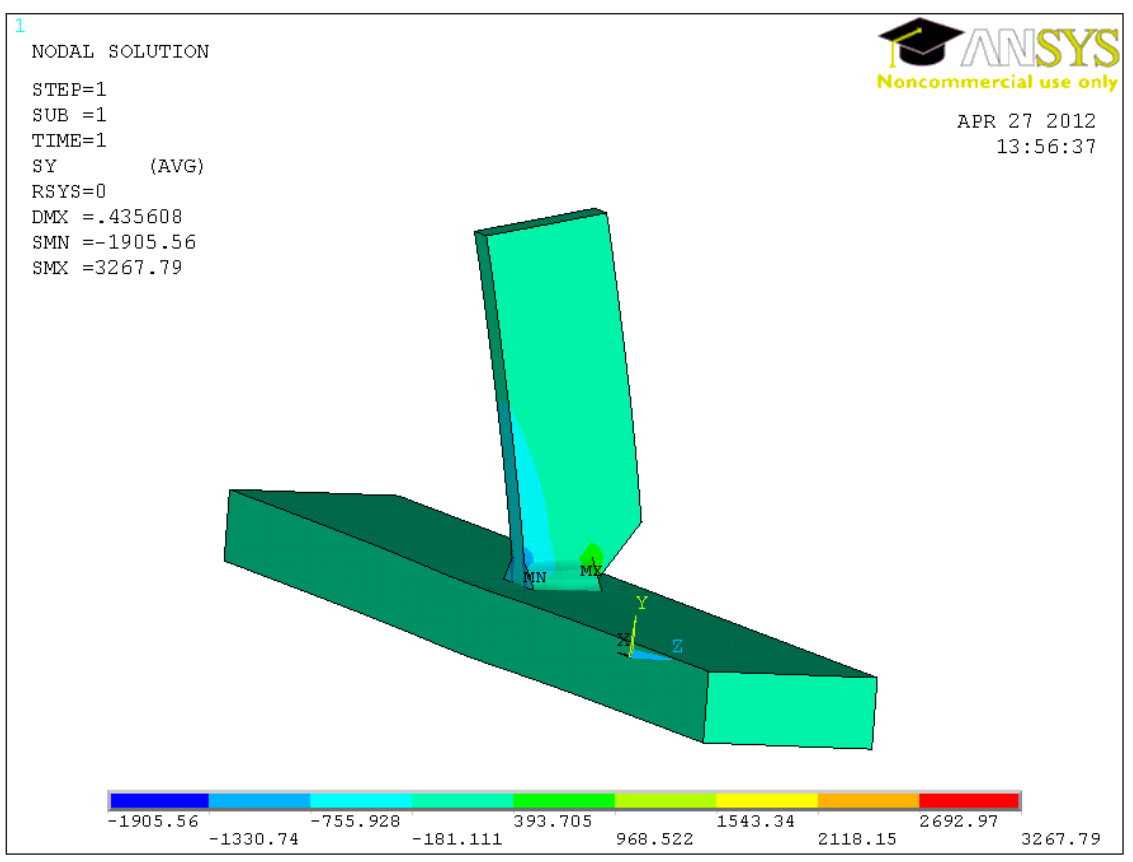

Figure 40: 3-D Cut-out Model Deflected Shape with Contours

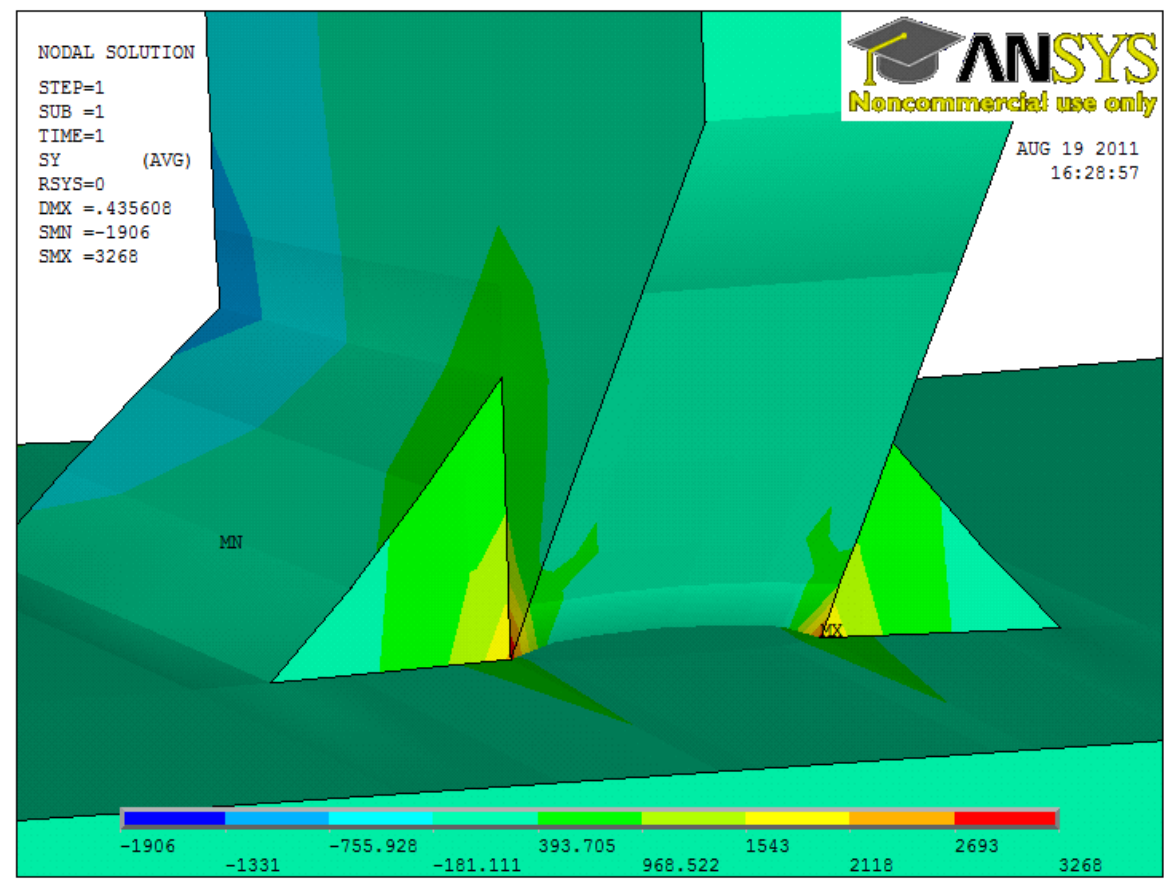

Figure 41: 3-D T-connection with Cut-out created with 20 Node Element 


\subsubsection{Preliminary Model Summary}

The stress concentrations in the preliminary models showed that the hot spots are dependent on the degree of constraint applied. The stress fields from the boundary conditions applied create large stress concentrations at the root and/or toe of the fillet weld. A highly meshed region must be applied at both locations, as a result. Accurate boundary conditions, including fillet weld shape, penetration, and residual stress, must be utilized for the correct results at the crack front.

\subsection{Results from Complete Local Model}

It was decided to develop the complete local models using the ANSYS Parametric Design Language, with the objective of making a useful model for multiple bridges. The boundary conditions for the model were extracted from Rostek's global model, and processed using a MATLAB routine to provide the necessary mapping from the global model to the local model boundaries. The model displacement boundary conditions were used as the loading for the complete local ANSYS model. Since the global model was constructed using plate elements, and the local model uses solid elements, it was necessary to interpolate the displacements from the plate element global model to the local model. Some care had to be exercised in this interpolation, since the node locations of the solid and plate models do not overlap. Some local stress concentrations resulted at the ends of the model where the boundary conditions were applied. These were quite localized, and were far enough away from the region of interest that they could be ignored. However, other extrapolation problems occurred in establishing the boundary conditions for the top flange that were somewhat more problematic. After several tries at interpolating between nodes across the top of the flange, where the large interpolation 
distances caused serious problems, it was decided that the only workable solution for the current model is to constrain the edges of the top flange only.

After simulating the response of the local model using the global model, it was observed that the top flange and stiffener materials overlapped on the diaphragm side. There are several ways to resolve this issue: add contact elements, couple degrees of freedom, or increase the gap between stiffener and flange. Contact elements would create a non-linear model that would increase the processing time significantly. It was felt that increasing the gap would compromise the model's fidelity. Consequently, it was decided to use coupling degrees of freedom. The disadvantage to using this approach was that the model had to be run twice. The first run was used to determine the elements that overlapped in the displaced state, and establish the degree of freedoms that needed to be coupled. The second run introduced coupling and provided the final results.

The actual boundary conditions from the previously constructed global model resulted in hot spots on the exterior stiffener. The results are shown in Figure 44. Figure 42 shows a cross-section of Girder A. Figure 43 shows a better location of Figure 44. The red circle is the location of the next Figure. All cut-out Figures are located at the red circle on Figure 43 unless otherwise specified. Originally, it was thought that the large tension stress concentrations would be on the side of the diaphragm cross-frame. This actually is not the case. 


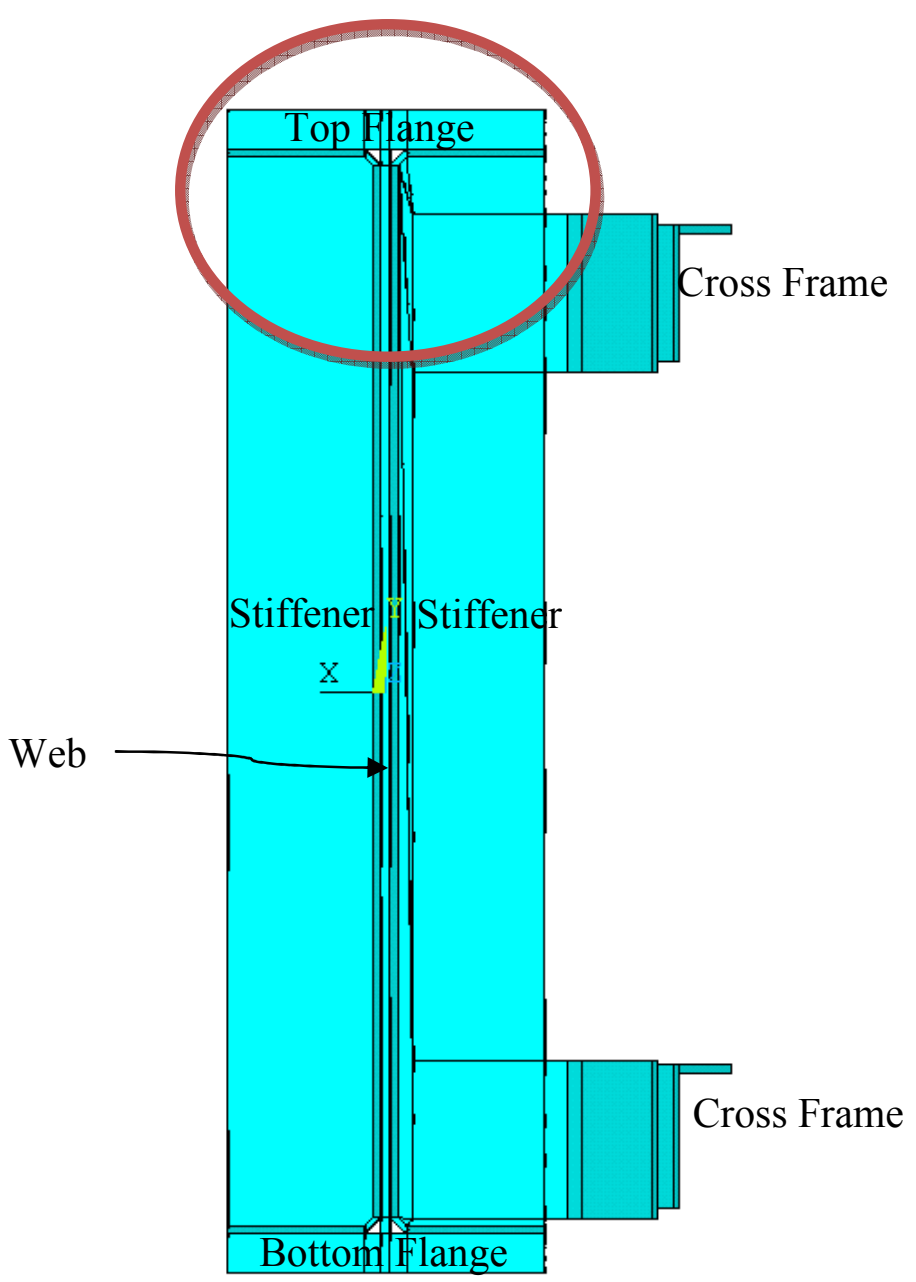

Figure 42: 2-D Cross-section of Girder A

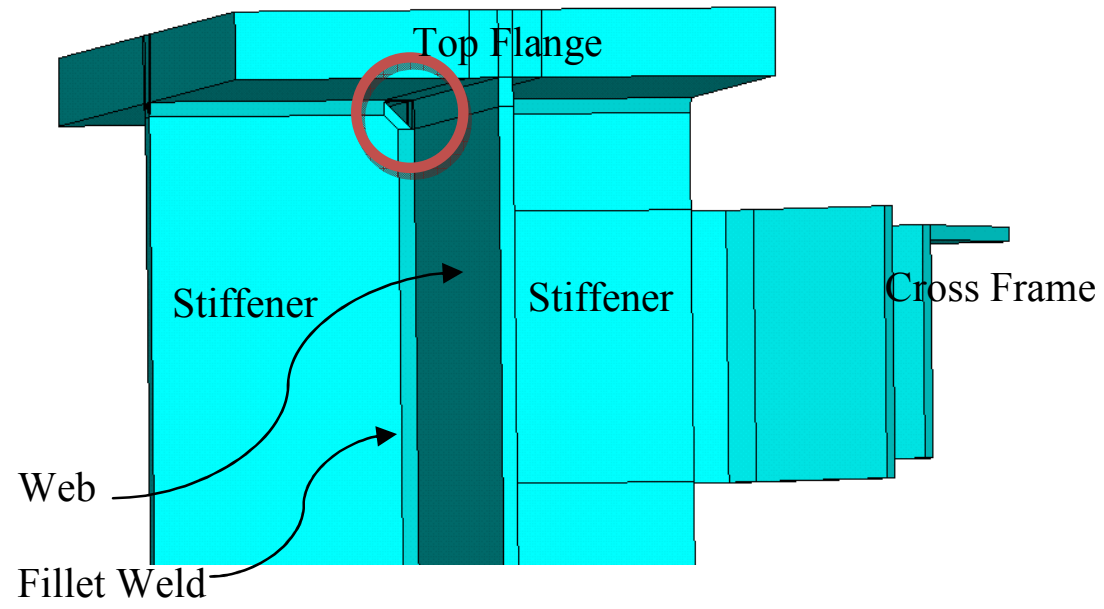

Figure 43: Zoomed Location of Stress Concentrated Cut-out 


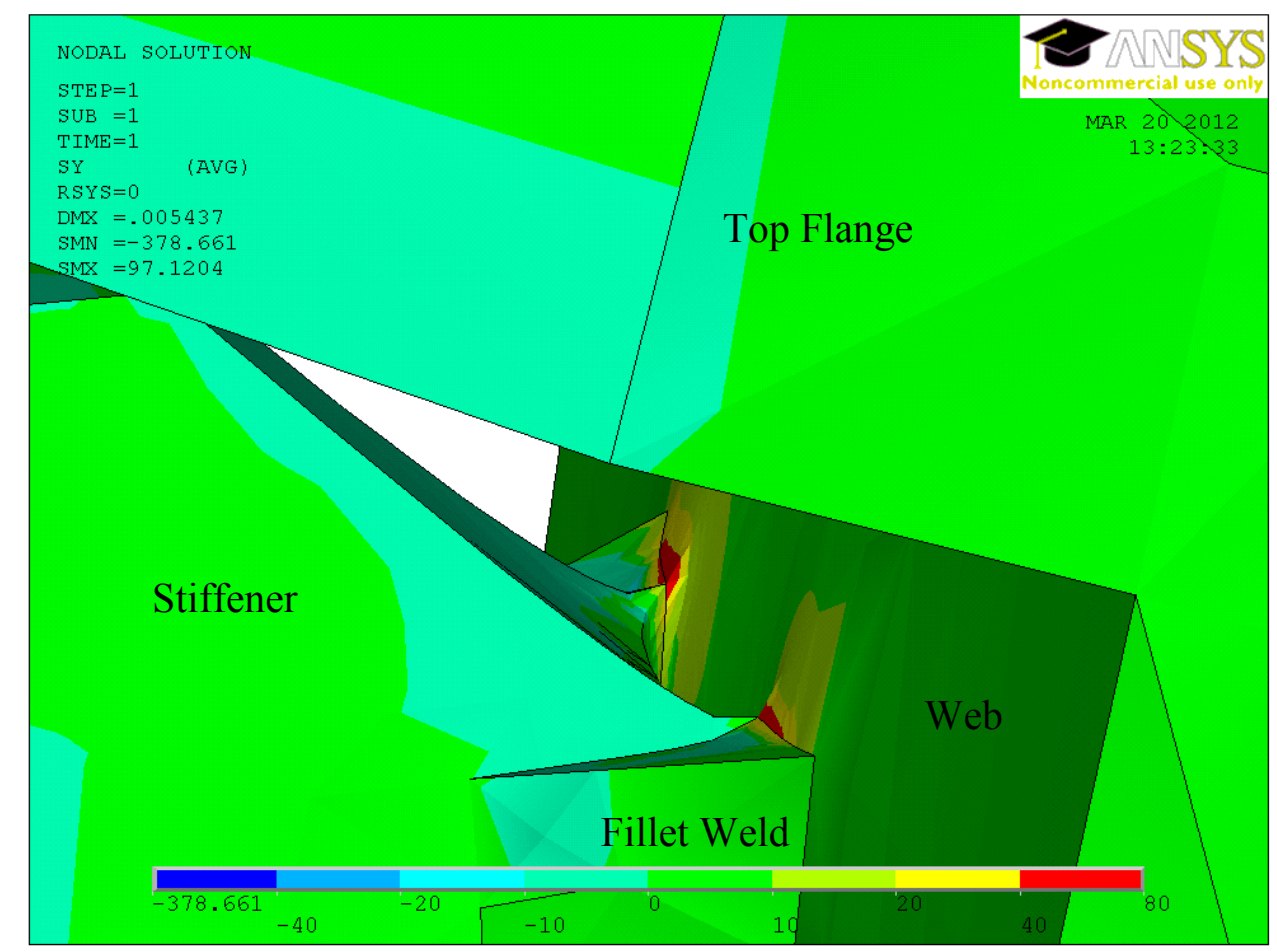

Figure 44: Cut-out of Exterior Stiffener

The largest tensile stress concentration occurs at the root of the fillet weld on the exterior stiffener, which was somewhat surprising. This condition is explained by examining the displacements of Rostek's global model. The displaced shape of the region of the global model corresponding to the complete local model, in Figure 29, shows that the top flange rotates toward the side of the diaphragm, leading to web distortion that tends to open the stiffener side, with the diaphragm connector plate being in compression. This accounts for the tension concentration on the exterior side. A comparison between local model and global model section was then conducted. The behavior of the local model was consistent with the observed displacement of the global model. The global model did show that the top angle was pushing and the bottom angle was pulling, shown in Figure 45. It also showed the bottom flange curved in Figure 46. 


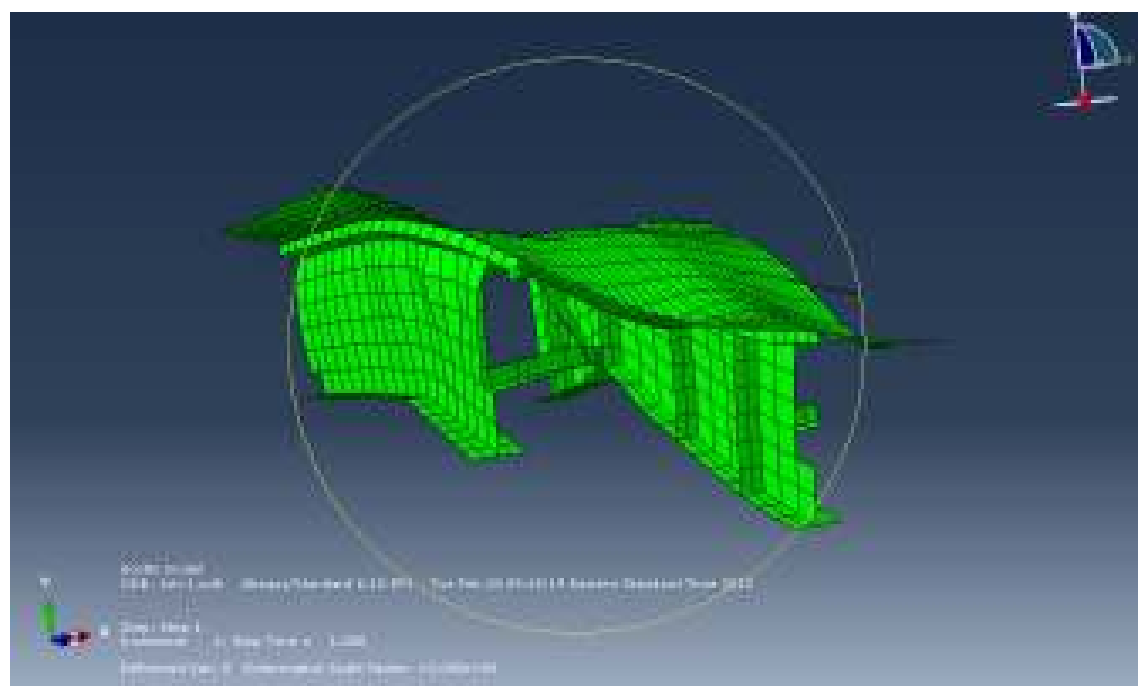

Figure 45: Global Results, Girder A is on Left

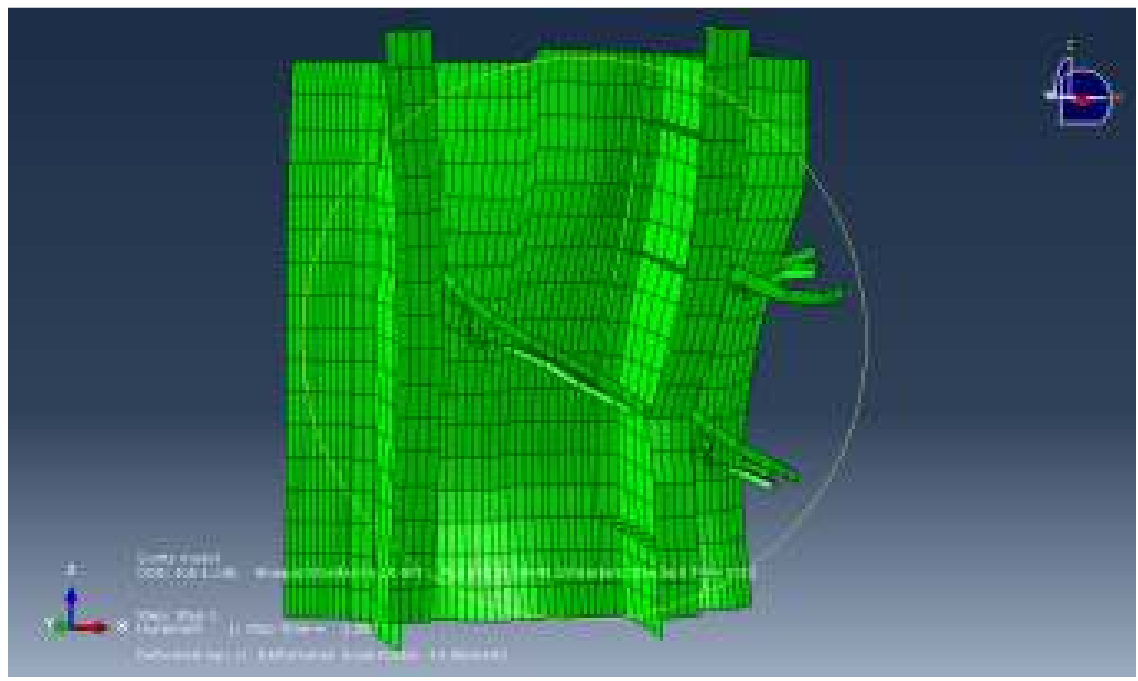

Figure 46: Bottom View of Global Results, Girder A is on Left

The complete partial model discovered that the stress concentration in the connector plate on the diaphragms is minimal. This would not have been discovered if the global model was not created. Initially, the stresses in the angles were hypothesized to be the largest. The idea was that one girder would displace due to the load, which would cause the diaphragm to pull/push the next. In actuality, the deck rotation is the primary motion causing such a displacement. This in turn causes the hot spots to be 
located on the exterior side of the girder at the root of the fillet weld. In this model, the side with the diaphragm had compression at the notch, while the exterior stiffener had tension.

The location of the load could be one reason for the low stresses in the diaphragm. The truck loading was applied on top of girder B directly adjacent to pier 10, causing maximum shear. A different stress concentration pattern is to be expected if the truck loading is located at a different position on the bridge. Since the support points at the skewed diaphragms do not translate vertically, the forces in the cross-frames can only be influenced by relative rotations of girders A and B. Higher stresses in the fillet welds of the connection plates might be observed if the load is placed at position that causes the maximum difference in rotation between girder $\mathrm{A}$ and girder $\mathrm{B}$.

Since the source of motion was not the diaphragms, stress intensity equations from previous literature, such as Roddis and Zhao (2003), cannot be used. Simplified models to explore how this factor could be found were tried.

\subsection{Results from a Partial Local Model}

The complete local model described in Section 4.2 utilizes all available information from the global model to construct the boundary conditions for the local model. This process can tend to be somewhat tedious, since matching the global and local models may involve interpolation of nodal displacement data between dissimilar model types. Therefore a partial local model is considered in this section. The objective of a partial local model is to replace the actual boundary conditions, which are tedious to construct, with a simplified set of boundary conditions that nonetheless capture the important behavior of the system. For example, Roddis and Zhao (2003) were able to use 
a partial local model because it was straightforward to construct the loading from the cross-frame members, which are primary members in the structure under consideration. Unfortunately, the geometry and loading of the multi-girder bridge with skewed crossframes over the supports is not particularly suitable for partial local models. The source of the difficulty is easily seen from the deformed section cut from the global model, and shown in Figure 45. Normally, the partial local model applies idealized displacements to the cross sections and then applies an appropriate compression or tension force through an adjoining member. For example, Roddis and Zhao (2003) fixed the top flange, assuming that the slab acts to restrain the motion of the girder's flange, and applied the cross-frame force as a direct tension load. Unfortunately, the loading mechanism for the multi-girder system under consideration is the slab itself. Moreover, the ends of the section undergo considerable rotation, so it is inaccurate to provide a short length of the girder with fixity at the ends of the segment. Since the cross-frames respond to the girder deformation, rather than applying the load to the girders, it is not at all clear what the correct magnitude of force to apply through the cross-frame members should be. Consequently, it was decided to apply a nominal load through the cross frame members to determine the nature of the local stress concentrations at the fillet weld ends. A one ksi traction was applied to the cross-frame extension in tension and compression to evaluate the feasibility of this modeling approach. There was no attempt to establish the correct magnitude, so any results of this approach are purely qualitative. Even if the magnitude had been correct, the results did not show the "hot spots" in the anticipated locations. On both sides of the web, one side of the notch was in tension and the other in compression as shown in Figure 47. This result is not surprising, because of the angle at which the 
cross-frame member exits the connection plate, and on the side of the plate that is in tension, a stress concentration exists at the root of the weld. However the result does not correspond to the complete local model. This indicates that a partial local model may be of limited value for this configuration.

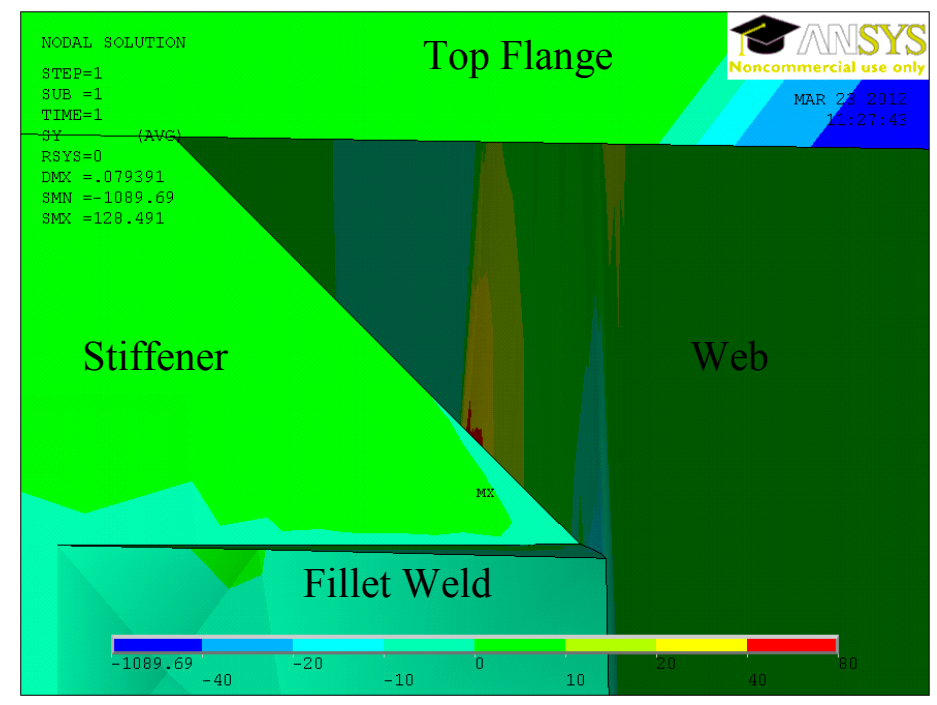

\section{Figure 47: Fixed Boundary Condition with Push-Pull Diaphragm Side}

Several additional simplified models were constructed in an attempt to find a partial local model that would provide results similar to the actual boundary conditions. One simplified boundary conditions involved rotating the top of flange and fixing the rest of the girder. It was thought that this might work since it is apparent from Figure 45 that the slab rotation was the driving force of the displacements. As shown in Figure 48, this model provided a much smaller stress intensity at the cut-out. 


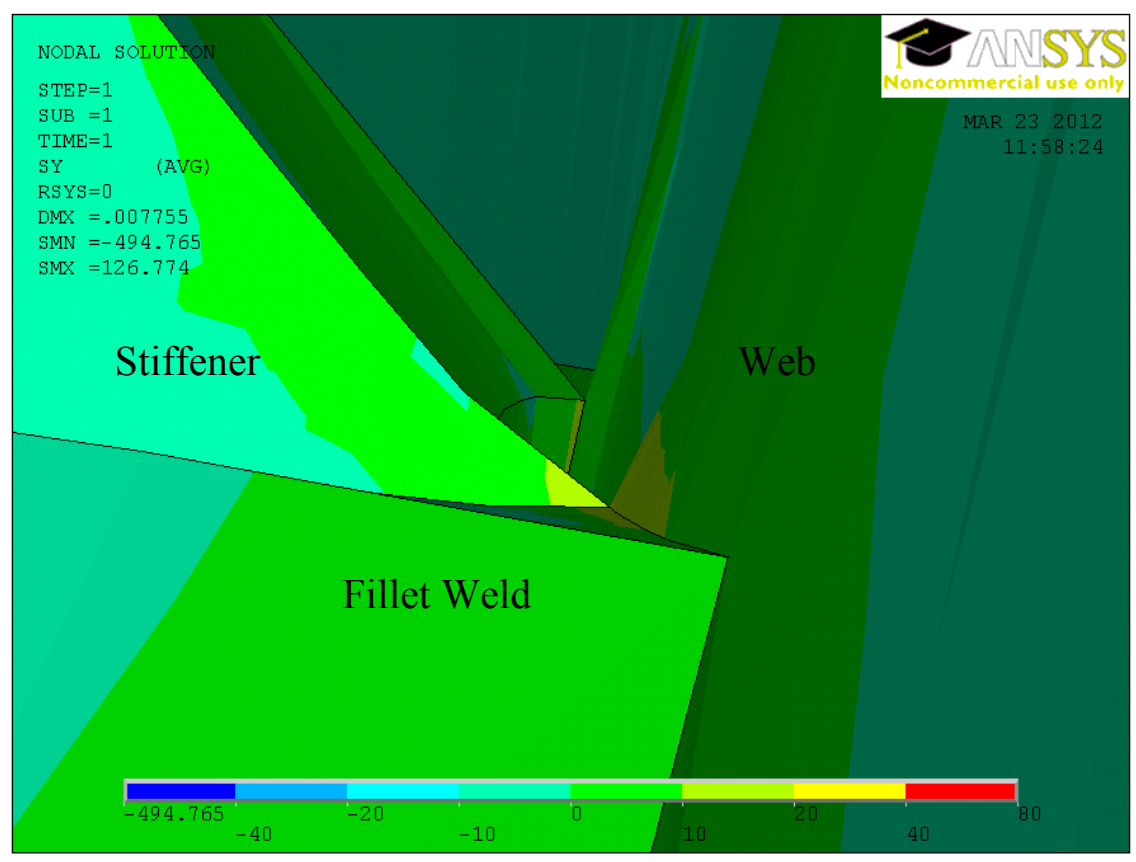

\section{Figure 48: Rotation of Top Flange Rest Fixed Diaphragm Side}

The last simplification was to use the correct displacements everywhere, except at the diaphragm but push and pull on the diaphragm. The stresses predicted using this model are shown in Figure 49 and are similar to those obtained from the fixed boundary conditions with the push and pull tractions. No other modified boundary conditions were tried. It was concluded that a truly partial local model was not feasible for the multigirder skewed configuration under consideration. 


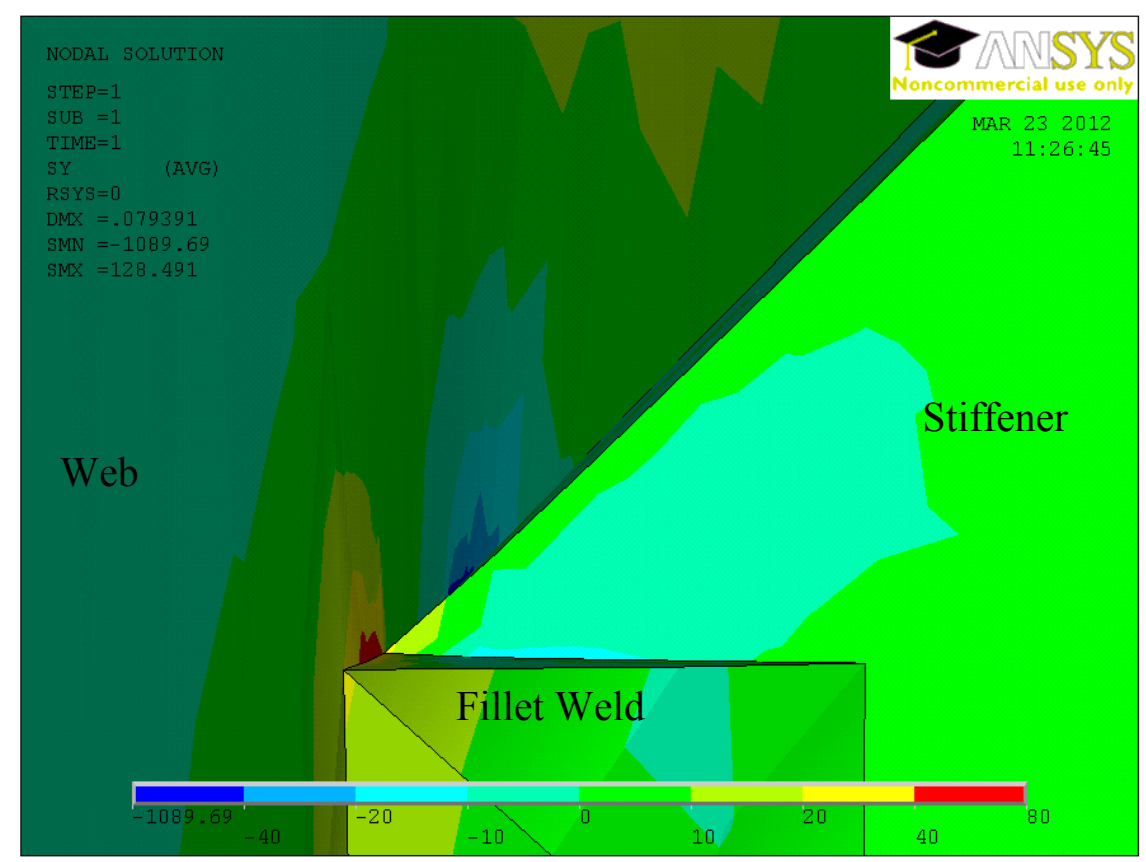

Figure 49: Actual Displacements on Cross Section with Push Pull on Diaphragm

\subsection{Web-Distortion Corrections}

As stated before, web distortion occurs because the web is allowed to rotate, between the flange and the connection plate cut-out. One remedial measure that has been recommended in past is to fasten the connection plate to the top flange, either by bolting or welding. Bolting is not a feasible retrofit procedure in most cases, so a fillet weld was added on the tension flange to see if this would reduce the stresses at the cut-out. The actual displacements from Girder A were used on this model. The resulting stress field indicates that this retrofit measure does, in fact, reduce the stress concentration significantly, as shown in Figure 50. 


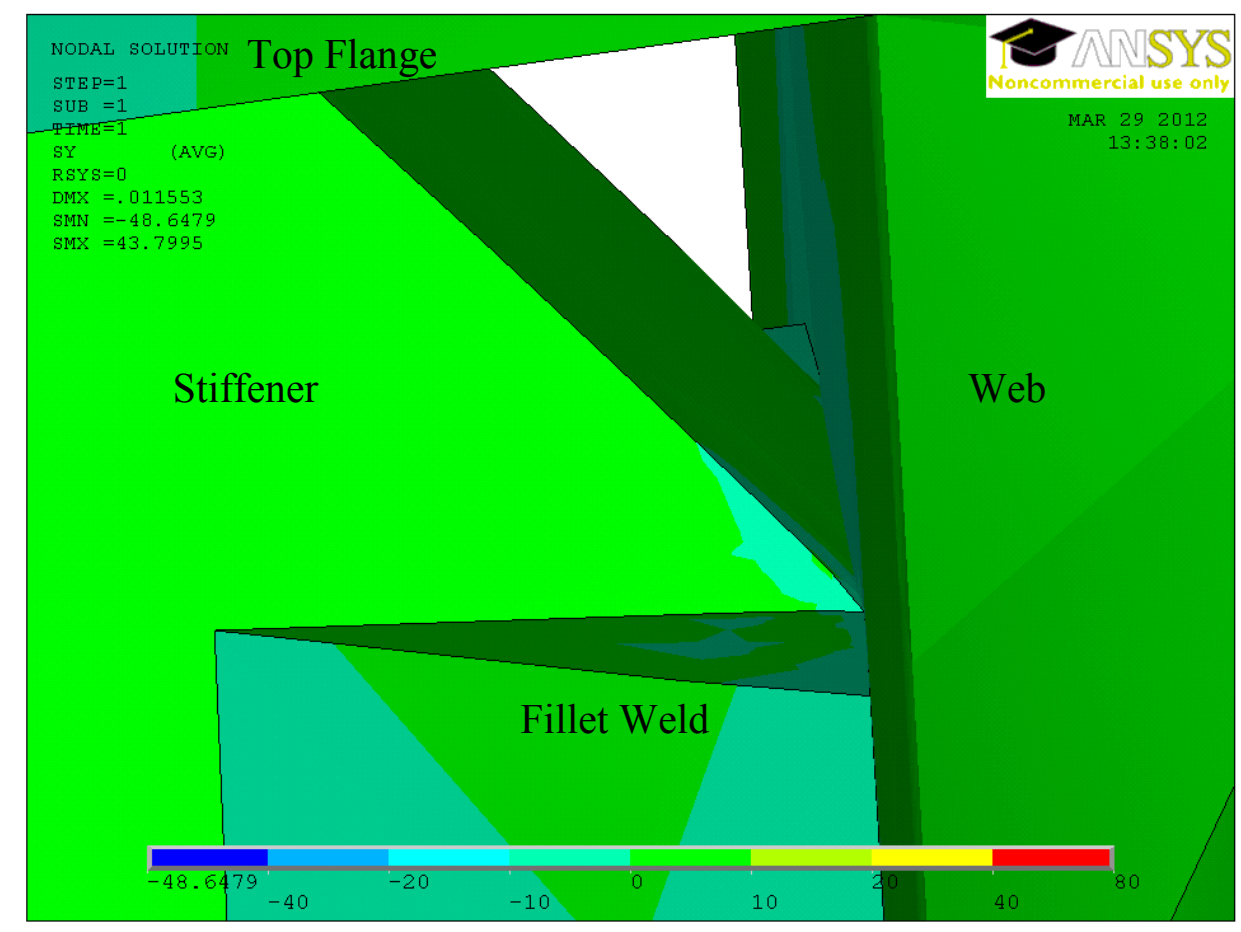

Figure 50: Modified Cut-Out

All the stresses in this model are relatively small. A high stress concentration at the edge of the flange where it connects with the stiffener on the diaphragm side was created. This was due to the flange constraint introduced by the welded connection plate displacements, at that point. Because of the imposed boundary conditions in the local model, the flange wants to separate from the stiffener but the added fillet weld does not allow it. It would have been beneficial to add the fillet weld in the global model and reexamine the results in the local model. Based upon this preliminary model, it does appear that the tension fillet weld will help eliminate the web distortion problem.

\subsection{Stress Intensity Factor}

For the three-dimensional geometry of the connection plate to web cutout, no simplified two-dimensional model is available that will properly calculate the stress 
intensity factor. Since there is no tabulated stress intensity factor equation for this specific geometry and loading, the correct stress intensity factor could not be determined without recourse to $\mathrm{J}$ integral calculations. In view of several limitations in the model, including the absence of residual stress effects, and uncertainty concerning the depth of weld penetration, it was decided that this calculation would be of limited value, and instead it was decided to search the literature for a stress intensity factor that appears to be somewhat suitable. After reviewing the available stress intensity factors in Tada et al (2000), one equation that used simplifications in geometry and in loading was selected as being most like the situation being modeled. This equation was used to provide a rough estimate of the stress intensity factor. Normally, the actual crack length and actual stress are used as inputs. Since, the actual crack length is unknown; the critical crack length will determine if the equations work accurately for the geometry and load case.

The plane strain fracture toughness will be used as the relevant condition for the critical stress intensity factor so the critical crack length can be found. This is applicable due to the fact that the plane strain fracture toughness is the minimum stress intensity factor at which a crack becomes unstable. The applied stress used is taken from the model output. The plane strain fracture toughness was found to be $148 \mathrm{MPa} \vee \mathrm{m}$ (Brnic et al, 2010) for A709 steel. Although A709 was not explicitly stated in the plans, it is representative of the steels used in this type of structure, so this was an acceptable assumption, given the approximate nature of the model. Two critical crack lengths were determined; one leading to a through web crack and another leading to a crack across the fillet weld These two sceneriors are the mostly likely to occur. 
In the tabulated case that was considered most appropriate, shown in Figure 51, the mode I stress intensity factor has the form: $\mathrm{K}_{\mathrm{I}}=1.1125 \sigma \sqrt{ } \mathrm{a}$. The crack is a combined mode crack, so $K_{I I}$ and $K_{I I I}$ factors are also tabulated for this case, but they are not considered here. The stress in the $\mathrm{x}$ direction was used when assuming that the crack extends through the web. The stress in the $\mathrm{z}$ direction was used when assuming the crack is a fillet crack. The stress outputs at the toe of the fillet weld provided by ANSYS is $\sigma_{x x}=12.358 \mathrm{ksi}$ and $\sigma_{z z}=14.925 \mathrm{ksi}$. The stress components at the root of the fillet weld predicted by ANSYS are $\sigma_{x x}=50.409 \mathrm{ksi}$ and $\sigma_{z z}=36.135 \mathrm{ksi}$.

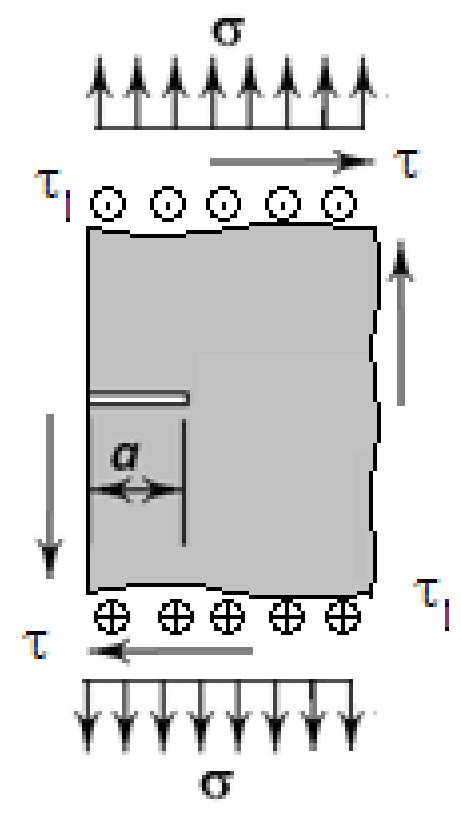

Figure 51: Stress Intensity Factor Case (Tada et al, 2000)

In the absence of residual stresses, the critical crack length would have to be 20.49 inches if the crack started at the toe and went through the web. It would be larger in this case if the crack started at the toe and cracked off the fillet weld. These values indicate that if a crack occurred, beginning at the toe, it would not reach a critical length 
until it has propagated beyond the point where the present calculations are valid. It is believed, based upon field observations that the critical crack length is considerably shorter, and suggests that this is not the correct equation for this failure event. The same simplified equation was used to evaluate critical crack lengths for cracks starting at the root as well, since that is the location of maximum calculated stress concentration in the local model studies. If the crack started at the root extending into the web, the critical length would be 3.52 inches. The crucial length for a fillet weld crack would be 1.81 inches. These values would seem more likely to be of the correct order of magnitude, but does not fully represent the complexity of the geometry. In order to further evaluate the stress intensity factor for this problem, it is necessary to introduce cracks of different types, and evaluate the three-dimensional problem using techniques such as a $\mathrm{J}$ integral, for different crack lengths. 


\section{Summary and Conclusions}

The global-local modeling approach has considerable promise for the evaluation of stress intensity factors in structural systems under complex loadings. This thesis investigated one particular scenario, in which a truck is located in a region that creates high shear in the web of the exterior girder near the site of an observed unstable crack. The complex interaction between girders introduced by the multi-girder system, and the skewed cross-frames over the piers makes simplified global models impractical, and it was necessary to use a full global-local modeling approach.

Originally, it was believed was that the diaphragms would cause the greatest web distortion due to the interaction of girders caused by vehicle loads. In this study, the skewed diaphragm over pier 10 actually experiences very low stresses, serving primarily to hold the girder in place instead of introducing the distortion. A major factor in this behavior is the fact that the girders cannot translate vertically at the location of the skewed diaphragm, so the diaphragm cannot serve the function of distributing vehicular loads between girders. The diaphragm hence acts as a secondary element, meaning that it is not directly involved with causing the stresses, at least under the loading considered. It was assumed that the tension or compression forces in the diaphragm's members were driving the stress at the cut-out, but due to this geometry and support conditions, the rotation of the flange was in fact driving it. The pins at the piers support the bridge all the way across. The original goal was to create multiple girder models to compare the stress concentrations with different diaphragm conditions. Since the slab controlled this stress at the pier location, it was decided not to use the other models that were created in 
the current study. Without comparing the local model and the global model, the mechanism controlling of the stresses at the connection would not have been properly understood.

The local model indicated that larger stress concentrations exist at the root of the fillet weld than at the toes. The location could have been affected by several assumptions that were made in this study. Specific weld information would have been helpful to acquire more accurate results. For example, the location of the most severe residual tension stresses, presumed to be at the toes, would have strongly influenced the location of the maximum tensile stresses. It was assumed that there was a crack between the stiffener and the web, but in reality, if a full penetration weld were used, this crack would not have been present, in which case there would be no stress concentration at the root. Even if there is no weld penetration, as assumed, the residual tension at the toes could cause residual compression at the root. The actual shape of the weld would have been helpful, but the only way to get that information would be to go to the site and measure it. In particular, a simplified shape was chosen for the weld end. For future modeling, the surface profile as well as the shape of the weld end is important. It was assumed it was a flat outside shape and a perpendicular end to the weld instead of a rounded outside and end edge. The shape of the weld and the residual stresses can be altered by the number of weld passes and the start and ends of the weld passes. It was assumed that the weld had no defects, but the weld could have defects that could cause the crack at the toe rather at the root. It is now assumed that if a flaw is present in the weld, the natural propagation would be through the web. The correct input into the local model, including boundary conditions, weld configuration details, and residual stresses, are essential for accurate 
results. For a multi-girder bridge, the best way to get accurate results using a local model is to use the deflection boundary condition obtained from a complete global model. Simplified load application and geometry for the local model were both attempted, but no models produced similar results as the complete local model for a multi-girder bridge configuration.

Since no simplified model were acceptable and there are no stress intensity equations for the exact geometry and loading, the precise stress intensity factor remains unknown for the particular complex three-dimensional geometry with complex loading boundary conditions modeled in this study. Although this study did not find a straightforward approach to solving this problem; it did however provide a further understanding of this problem that will be useful in developing improved approaches in the future. The understanding of the accuracy of boundary condition, modeling assumptions, and the extent of fracture mechanics in ANSYS are all vital for future work. 


\section{Future Work}

Although the stress intensity factor was not estimated for a complex threedimensional geometry with complex loading, this study gave some great insight into this topic. Several recommendations are proposed that will provide assistance in developing improved models for the problem. One of the biggest issues was mapping correct boundary conditions between the global and local models, including the incompatibilities between a plate model and a solid model. The element type incompatibilities produced a displacement that oscillated about the actual displacement at the constrained boundaries. This in turn caused an incorrect stress pattern at some locations. Although these regions are generally far enough from the detail of interest to not cause any problems with the solution, some locally high stresses were obtained that made the graphical display of the stress contours of interest somewhat more difficult. It is suggested that more time be spent on understanding this issue and determining how to reduce those effects. One way to accomplish this would be to use sub-structuring, which embeds the local model into the global model. Multiple element types, like plate and solid elements, could be used in this model although a more suitable model might be constructed using a single element type for both global and local models, possibly with extensive substructuring to maintain the global model at a reasonable size. A Global-Local-Local model would improve upon the Global Local accuracy by embedding a heavily meshed local model in a larger local model, which then in turn is located in the global model. Such a second order refined local model might be more amenable to including subtle information such as weld shape 
and weld end conditions accurately. Correct boundary conditions and modeling information are vital for an accurate model.

As a prelude to further global-local modeling, accurate field data should be collected on bridges of this type that properly identifies the shape, and penetration of the fillet welds. Further, the magnitudes and distribution of the residual stresses for the fillet weld would provide very useful information. Finally, a survey of observed flaws in the welds at the critical regions would provide information that could be used to provide initial crack data for the local models. As stated in the conclusion, this information could significantly influence the results by increasing or relieving the stresses. After doing the main study, it seemed that some field research would have been helpful not only for the weld information but for accuracy of loading. A major influence not considered in the current study is the presence of thermal loading contributed by expansion and contraction of the slab relative to the girders. It is thought that this expansion and contraction may have the potential to produce distortion stresses of the same order of magnitude as any produced by vehicular loads. The thermal effects would have been a good addition to the global models. This could be a major contributor fatigue cracking that has not been examined fully. This study only addressed mechanical vehicle loading, which was only one static truck load. This loading did not cause a high enough stress to create a fatigue crack. It is suggested to increase the static load by $30 \%$ to include the effect of dynamic loading. In particular, the loaded location did not produce very large stresses in the cross-frame members. Because of the skewness of the bridge, increasing the relative rotations between girders $\mathrm{A}$ and $\mathrm{B}$ appears to be the most feasible way to increase the 
cross-frame stresses. Hence, it is also recommended to move the vehicle load to the location on the bridge that create the largest rotational difference between girder A and B. Another interesting study would be to create a small crack into the local model at the toe of the fillet weld and apply different boundary conditions to investigate crack tip stresses. This would result in a better understanding on how the cracks form with this specific geometry. Once the critical boundary conditions are found, a method could be attempted to fix this issue, like an addition of a fillet weld on the tensile flange. Originally, this weld was absent to decrease fatigue effects. The stress concentration developed from the ANSYS local model showed a significant decrease of stress at the cut-out with the additional fillet weld. It should be experimentally determined if the additional fillet causes better or worse fatigue stress than web-distortion.

Any data collected from field studies or numerical experiments using the global model should be used to provide improved boundary conditions for the local model. If displacement boundary conditions are used, as in this study, it is proposed that a refined global model be produced in the region of the local model to provide more complete displacement boundary conditions for the local model. If strains are used instead, stresses, calculated from these strains, may then determine the stress intensity factor. It was found that ANSYS did not produce the stress intensity factor in three-dimensional easily. Most literature reviewed during the course of this study used ANSYS to find the far field stresses and use those as inputs into a specialized fracture analysis program. This technique or producing the whole model in a generalized fracture analysis program is suggested. Finally, several programs are available that feature built-in global-local 
modeling capability, and permit adaptive remeshing for crack growth modeling. Such models would be invaluable for future studies. 


\section{Bibliography}

Alam, M., \& Wahab, M. (2005). Modeling the Fatigue Crack Growth and Propagation Life of a Joint of Two Elastic Materials using Interface Elements. International Pressure Vessels and Piping 82, 105-113.

Aljutaili, Dhari S. (2007) Distortion-Induced Fatigue Cracking of Girder-to-Crossbeam Connection, M.S. Thesis, Department of Mechanical Engineering, Oregon State University, Corvallis OR.

Anderson, T.L. (1995). Fracture Mechanics: Fundamentals and Application $2^{\text {nd }}$ ed. New York: CRC Press, Inc.

Altus, E., Tirosh, J., \& Yifrach, Y. (1992). A New Method For Evaluating Fracture Toughness of Brittle Materials. International Journal of Fracture 58, 211-222.

Bakker, A. (1992). Three-Dimensional Constraint Effects on Stress Intesnity Distribution in Plate Geometeries with Through Thickness Cracks. Fatigue and Fracture of Engineering Material Structures 15, 1051-1069.

Barsom, J. M., \& Rolfe, S. T. (1999). Fracture and Fatigue Control in Structures: Applications of Fracture Mechanics. Woburn: Butterworth Heinemann.

Barsoum, R. S. (1976). On the Use of Isoparametric Finite Elements in Linear Fracture Mechanics. International Journal for Numerical Methods in Engineering, 10, 25-37.

Barsoum, R. S. (1977). Triangular Quarter-Point Elements as Elastic and Perfectly Plastic Crack Tip Elements. International Journal for Numerical Methods in Engineering, 11, 85-98.

Berglund, Evan and Schultz, Arturo. (2006). Girder Differential Deflection and Distortion-Induced Fatigue in Skewed Steel Bridges. Journal of Bridge Engineering, ASCE, Vol. 11, No. 2, pp. 169-177.

Brnic, J., Canadija, M., Turkalj, G., \& Lanc, D. (2010). Structural Steel ASTM A709Behavior at Uniaxial Tests Conducted at Lowered and Elevated Temperatures, ShortTime Creep Response, and Fracture Toughness Calculation. Journal of Engineering Mechanics, 1083-1089.

Carpinteri, A. (1991). Stress-Intensity Factors for Semi-Elliptical Surface Cracks Under Tension Or Bending. Engineering Fracture Mechanics 38 , 327-334.

Chang, J.H., \& Wu, W.H., (2011). Using M-integral for Multi-cracked Problems Subjected to Nonconservative and Nonuniform Crack Surface Tractions. International Journal of Solids and Structures 48, 2605-2613. 
Chang, K.H., \& Lee, C.H. (2009). Finite Element Analysis of the Residual Stresses in T-Joint Fillet Welds Made of Similar and Dissimilar Steels. Int J Adv Manufacuturing Technology 41 , 250-258.

Chung, W., Liu, J., and Sotelino, E. D., (2006). Influence of Secondary Elements and Deck Cracking on the Lateral Load Distribution of Steel Girder Bridges, Journal of Bridge Engineering, ASCE, 11, 2, 178-187.

Connor, R. J., and Fisher, J. W. (2006) . Identifying Effective and Ineffective Retrofits for Distortion Fatigue Cracking in Steel Bridges Using Field Instrumentation. Journal of Bridge Engineering, ASCE, Vol. 11, No. 6, pp. 745-752.

Cousins, T. E., Stallings, J. M., Lower, D. A., and Stafford, T. E., (1998,. Field Evaluation of Fatigue Cracking in Diaphragm-Girder Connections, Journal of Performance of Constructed Facilities, ASCE, Vol. 12, No. 1, pp. 25-32.

DePiero, A. H., Paasch, R. K., and Lovejoy, S. C. (2002). Finite-Element Modeling of Bridge Deck Connection Details. Journal of Bridge Engineering, ASCE, Vol. 7, No. 4, pp. 229-235.

Dodds Jr, R., \& Reed, D. (1984). Elastic-Plastic Response of Tensile Panels Containing Short Center Cracks. Computational Fracture Mechanics- Nonlinear and 3-D Problems , 25-34.

Ferreira, J. M., \& Branco, C. M. (1991). Influence of Fillet Weld Joint Geometry on Fatigue Crack Growth. Theoretical and Applied Fracture Mechanics 15, 131-142.

Fisher, J. W. (1984). Fatigue and Fracture in Steel Bridges: Case Studies. New York: John Wiley \& Sons, Inc.

Fisher, J. W., Jin, J., Wagner, D. C., and Yen, B. T. (1990) Distortion induced Fatigue Cracking in Steel Bridges, NCHRP Report No. 336, National Research Board, Washington, DC.

Fraser, R. E. K., Grondin, G. Y., and Kulak, G. (2000) Behavior of Distortion-induced Fatigue Cracks in Bridge Girders, Structural Engineering Report No. 235, Department of Civil and Environmental Engineering, University of Alberta, Edmonton.

$\mathrm{Fu}$, Kuan-Chen and Lu, Feng. (2003). Nonlinear Finite-Element Analysis for Highway Bridge Superstructures. Journal of Bridge Engineering, ASCE, Vol. 8, No. 3, pp. 173179.

Guo, W., She, C., Zhao, J., \& Zhang, B. (2006). Advances in Three-Dimensional Fracture Mechanics. Key Engineering Material 312, 27-34.

Henshell, R. D. and Shaw, K. G. (1975) "Crack Tip Elements are Unnecessary," International Journal for Numerical Methods in Engineering, 9, 495-507. 
Kujawski, D., \& Stoychev, S. (2008). Crack-tip Stresses and Their Effect on Stress Intensity Factor for Crack Propagation. Engineering Fracture Mechanics 75 , 24692479.

Lovejoy, S. C., (2003) "Determining Appropriate Fatigue Inspection Intervals for Steel Bridge Members," Journal of Bridge Engineering, ASCE, Vol. 8, No. 2, pp. 66-72.

Lydzinski, John C. (2006). Finite Element Modeling of a Horizontal Curved Bridge. M.S. Thesis, Department of Civil and Environmental Engineering, University of Virginia, Charlottesville, VA.

Massarelli, Peter J. (1997). Fatigue Reliability of Steel Highway Bridge Details, PhD. Thesis, Department of Civil and Environmental Engineering, University of Virginia, Charlottesville, VA.

Miranda, A., Meggiolaro, M., Castro, J., Martha, L., \& Bittencourt, T. (2003). Fatigue Life and Crack Path Predictions in Generic 2-D Structural Components. Engineering Fracture Mechanics 70 , 1259-1279.

Miller, J. E. (2008) Strain Instrumentation of a Multi-span Curved Girder Bridge, M.S. Thesis, Department of Civil and Environmental Engineering, University of Virginia, Charlottesville, VA.

Munse, William H. (1964) Fatigue of Welded Steel Structures, Welding Research Council, New York, NY.

Patricio, M., \& Mattheij, R. M. (n.d.). Crack Propagation Analysis. Retrieved February 23, 2012, from http://www.win.tue.nl/analysis/reports/rana07-23.pdf

Roddis, W. K., \& Zhao, Y. (2003). Finite-Element Analysis of Steel Bridge DistortionInduced Fatigue. Journal of Bridge Engineering ASCE , 259-266.

Rostek, S. R., (2010), Finite Element Analysis of Distortional Web Stresses in a Skewed Plate Girder Bridge, M. S. Thesis, Department of Civil and Environmental Engineering, University of Virginia, Charlottesville, VA.

Schijve, J. (2001). Fatigue of Structures and Materials. Boston: Kluwer Acedemic Publishers.

Shih, C. F., Moran, B., and Nakamura, T (1986) Energy Release Rate Along a Threedimensional Crack Front in a Thermally Stressed Body. International Journal of Fracture, 30, 79-102.

Simons, D.C., (2005), AASHTO LRFD Impact upon Bridge Stiffness and Strength, M.S. Thesis, University of Virginia, Charlottesville. 
Stallings, J. M., Cousins, T. E., and Tedesco, J. W. (1996) Fatigue of Diaphragm Girder Connections, Final Report 930-307, Alabama Department of Transportation, performed at Auburn University. Auburn, AL.

Stallings, J. M., Cousins, T. E., and Tedesco, J. W. (1997). Fatigue of DiaphragmGirder Connections. Transportation Research Record 1594, Paper No. 970404.

Suresh, S. (1998) Fatigue of Materials, Cambridge University Press, Cambridge, U.K.

Tada, H., Paris, P. C., \& Irwin, G. R. (3rd ed. 2000). The Stress Analysis of Cracks Handbook. New York: The American Society of Mechanical Engineers.

Tedesco, J. W., Stallings, J. M. and Tow, D. R. (1995). Finite Element Method Analysis of Bridge Girder-Diaphragm Interaction. Computers and Structures, Vol. 56, No. 2/3, pp. 461-473.

Teng, T.-L., Fung, C.-P., Chang, P.-H., \& Yang, W.-C. (2001). Analysis of Residual Stresses and Distortions in T-Joint Fillet Welds. International Journal of Pressure Vessels and Piping 78, 523-538.

Tilley, M. (2004) Dynamic analysis and testing of a curved girder bridge, M. S. Thesis, University of Virginia, Charlottesville, VA.

Turnage, R. (2007) Vibration Testing of the Wolf Creek Curved Girder Bridge, M.S. Thesis, Civil and Environmental Engineering Department, University of Virginia, Charlottesville, VA.

Xanvhakos, P. (1994). Theory and Design of Bridges. New York: John Wiley \& Sons, Inc.

Yazdani, N., \& Albrecht, P. (1990). Probabilistic Fracture Manchanics Application to highway Bridges. Engineering Fracture Mechanics 37, 969-985.

Zhao, Y. and Roddis, W.M. (2003). Finite Element Study of Distortion-Induced Fatigue in Welded Steel Bridges. Transportation Research Record 1845, Paper No. 03-3245. 


\section{Appendix}

\subsection{ANSYS Code}

KEYW,PR_SET,1

KEYW,PR_STRUC,1

KEYW,PR_THERM,0

KEYW,PR FLUID,0

KEYW,PR_ELMAG,0

KEYW,MAGNOD,0

KEYW,MAGEDG,0

KEYW,MAGHFE,0

KEYW,MAGELC,0

KEYW,PR MULTI,0

KEYW,PR_CFD,0

/GO

/PREP7

ET,1,solid187,

KEYOPT,1,6,0,

MPTEMP,,,,,,,

MPTEMP, 1,0 ,

MPDATA,EX,1,29000,

*afun,deg

$\mathrm{bf}=14$

$\mathrm{bft}=1.75$

$\mathrm{tft}=1.75$

$\mathrm{wt}=.375$

$\mathrm{wh}=48$

length $=21.75^{*} 2$

$\mathrm{sl}=\mathrm{bf} / 2-\mathrm{wt} / 2$

snotch $=.75$

$\mathrm{st}=1$

fillet $=.375$

dp $1=1$

$\mathrm{dp} 2=1$

$\mathrm{dtp} 1=2+7 / 8$

$\mathrm{dbp} 2=0.625$

ptl1 $=7$

pt12=7

plt $1=.375$

plt2 $=.375$

pta $1=7$

pta2 $=7$ 


$$
\begin{aligned}
& \text { lbent } 1=5 \\
& \text { lbent } 2=5 \\
& \text { ang } 1=40 \\
& \text { ang } 2=40 \\
& \operatorname{cd} 1=15 / 16 \\
& \mathrm{cl} 1=5 \\
& \text { ct } 1=.375 \\
& 1 \mathrm{sc}=6 \\
& \mathrm{ssc}=4 \\
& \mathrm{dftf}=3+3 / 8 \\
& \mathrm{dfbtf}=7+1 / 8 \\
& \text { co1 }=\text { cl1-lbent } 1+\mathrm{cd} 1 \\
& \text { ctop } 1=\text { wh/2-dftf } \\
& \text { cang1 }=\text { ctop } 1 \text {-ct } 1 \\
& \text { cbot } 1=\text { ctop } 1-1 s c \\
& \text { ctop } 2=w h / 2-d f b t f \\
& \operatorname{cbot} 2=\operatorname{ctop} 2+1 \mathrm{sc} \\
& \text { cang2=ctop2-ct1 } \\
& \mathrm{q}=\text { lbent } 1 \text {-col } \\
& \mathrm{h}=\mathrm{wh}+\mathrm{bft}+\mathrm{tft} \\
& \mathrm{dsb}=\mathrm{h} / 2 \text {-bft- } .001 \\
& \mathrm{dst}=\mathrm{h} / 2 \text {-tft-.001 } \\
& \mathrm{d}=\mathrm{wt} / 2+.001 \\
& \mathrm{ds}=\mathrm{sl}+\mathrm{wt} / 2 \\
& \mathrm{dtf}=\mathrm{h} / 2-\mathrm{tft} \\
& \mathrm{dbf}=\mathrm{h} / 2-\mathrm{bft} \\
& \mathrm{dttf}=\mathrm{h} / 2 \text {-tft-fillet } \\
& \mathrm{dbtf}=\mathrm{h} / 2 \text {-bft-fillet } \\
& \mathrm{tsn}=\mathrm{h} / 2 \text {-tft-snotch } \\
& \text { bsn }=h / 2 \text {-bft-snotch } \\
& \mathrm{fd}=\mathrm{wt} / 2+\text { snotch } \\
& \text { lf=length/2-fillet-st } / 2 \\
& \text { ls }=1 \mathrm{f}+\text { fillet } \\
& 1 s s=1 s+s t \\
& \text { lff }=\text { lss }+ \text { fillet } \\
& \mathrm{f}=\mathrm{wt} / 2+\text { fillet } \\
& \mathrm{pd} 1=\mathrm{wt} / 2+\mathrm{dp} 1 \\
& \mathrm{pd} 2=\mathrm{wt} / 2+\mathrm{dp} 2 \\
& \text { pd12=pd1 } 1+\text { ptl1 } \\
& \operatorname{pd} 22=\text { pd } 2+\text { ptl } 2
\end{aligned}
$$




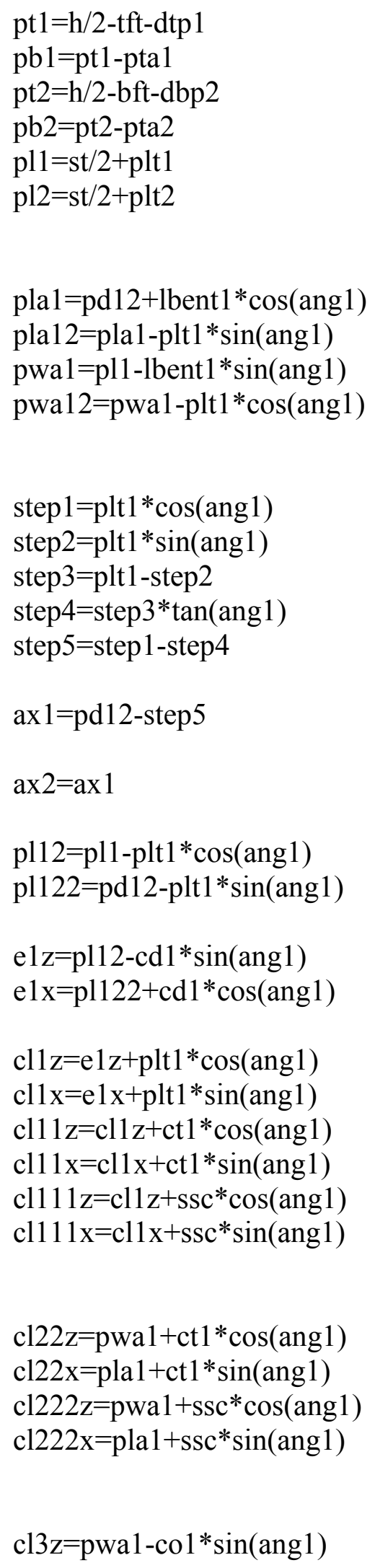




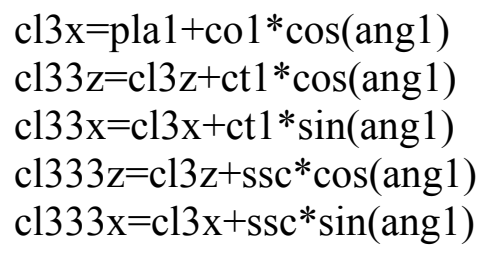

k, 1,bf/2,-h/2,length/2, $\mathrm{k}, 2, \mathrm{fd},-\mathrm{h} / 2$, length/2, $\mathrm{k}, 3, \mathrm{wt} / 2,-\mathrm{h} / 2$,length/2, k,4,-wt/2,-h/2,length/2, $\mathrm{k}, 5,-\mathrm{fd},-\mathrm{h} / 2$, length/2, $\mathrm{k}, 6,-\mathrm{bf} / 2,-\mathrm{h} / 2$, length/2, k, $7,-$ bf $/ 2$, -dbf,length/2, k,8,-fd,-dbf,length/2, k,9,-wt/2,-dbf,length/2, k, 10,wt/2,-dbf,length/2, $\mathrm{k}, 11, \mathrm{fd}$,-dbf,length/2, $\mathrm{k}, 12, \mathrm{bf} / 2$,-dbf,length/2, k, 13,wt/2,-bsn,length/2, k, 14,-wt/2,-bsn,length/2, $\mathrm{k}, 15$,wt/2,tsn, length/2, $\mathrm{k}, 16$,-wt/2,tsn,length/2, $\mathrm{k}, 17, \mathrm{bf} / 2$,dtf,length/2, $\mathrm{k}, 18, \mathrm{bf} / 2, \mathrm{~h} / 2$,length/2, $\mathrm{k}, 19, \mathrm{fd}, \mathrm{h} / 2$, length/2, $\mathrm{k}, 20, \mathrm{wt} / 2, \mathrm{~h} / 2$, length/2, $\mathrm{k}, 21,-\mathrm{wt} / 2, \mathrm{~h} / 2$, length/2, k,22,-fd,h/2,length/2, $\mathrm{k}, 23,-\mathrm{bf} / 2, \mathrm{~h} / 2$, length/2, $\mathrm{k}, 24,-\mathrm{bf} / 2$,dtf,length/2, k,25,-fd,dtf,length/2, $\mathrm{k}, 26$,-wt/2,dtf,length/2, $\mathrm{k}, 27$,wt/2,dtf,length/2, $\mathrm{k}, 28$,fd,dtf,length/2,

kgen, 2, 1,28, 1,0,0,-lf, kgen, $2,1,28,1,0,0,-1 \mathrm{~s}$, kgen, $2,1,28,1,0,0,-1 s s$, kgen, 2, 1,28,1,0,0,-1ff, kgen, 2, 1,28,1,0,0,-length,

k,169,ds1,-dsb,-st/8 


$$
\begin{aligned}
& \text { k,170,dsl,-dbtf,-st/2 } \\
& \text { k,171,dsl,-dbtf,st/2 } \\
& \text { k,172,fd,-dsb,-st/8 } \\
& \text { k,173,fd,-dbtf,-st/2 } \\
& \text { k,174,fd,-dbtf,st/2 } \\
& \text { k,175,f,-bsn,-st/2 } \\
& \text { k,176,f,-bsn,st/2 } \\
& \text { k,177,d,-bsn,-st/8 } \\
& \text { k,178,dsl,dst,-st/8 } \\
& \text { k,179,dsl,dttf,-st/2 } \\
& \text { k,180,dsl,dttf,st/2 } \\
& \text { k,181,fd,dst,-st/8 } \\
& \text { k,182,fd,dttf,-st/2 } \\
& \text { k,183,fd,dttf,st/2 } \\
& \text { k,184,f,tsn,-st/2 } \\
& \text { k,185,f,tsn,st/2 } \\
& \text { k,186,d,tsn,-st/8 } \\
& \text { k,187,-ds1,-dsb,-st/8 } \\
& \text { k,188,-dsl,-dbtf,-st/2 } \\
& \text { k,189,-ds1,-dbtf,st/2 } \\
& \text { k,190,-fd,-dsb,-st/8 } \\
& \text { k,191,-fd,-dbtf,-st/2 } \\
& \text { k,192,-fd,-dbtf,st/2 } \\
& \text { k,193,-f,-bsn,-st/2 } \\
& \text { k,194,-f,-bsn,st/2 } \\
& \text { k,195,-d,-bsn,-st/8 } \\
& \text { k,196,-ds1,dst,-st/8 } \\
& \text { k,197,-dsl,dttf,-st/2 } \\
& \text { k,198,-dsl,dttf,st/2 } \\
& \text { k,199,-fd,dst,-st/8 } \\
& \text { k,200,-fd,dttf,-st/2 } \\
& \text { k,201,-fd,dttf,st/2 } \\
& \text { k,202,-f,tsn,-st/2 } \\
& \text { k,203,-f,tsn,st/2 } \\
& \text { k,204,-d,tsn,-st/8 } \\
& \text { k,211,--pd12,pt1,pl1 } \\
& \text { k,212,-pd12,pb1,pl1 } \\
& \text { k,205,-pd1,pt1,pl1 } \\
& \text { k,206,-pd1,pb1,pl1 } \\
& \text { k,207,-ax1,pt1,st/2 } \\
& \text { k,208,-ax1,pb1,st/2 }
\end{aligned}
$$




$$
\begin{aligned}
& \text { k,213,-pd2,-pt2,pl2 } \\
& \text { k,214,-pd2,-pb2,pl2 } \\
& \text { k,215,-ax2,-pt2,st/2 } \\
& \text { k,216,-ax2,-pb2,st/2 } \\
& \text { k,217,-pd2,-pt2,st/2 } \\
& \text { k,218,-pd2,-pb2,st/2 } \\
& \text { k,219,-pd22,-pt2,pl2 } \\
& \text { k,220,-pd22,-pb2,pl2 } \\
& \text { k,221,-ds1,pt1,st/2 } \\
& \text { k,222,-dsl,pb1,st/2 } \\
& \text { k,223,-dsl,-pt2,st/2 } \\
& \text { k,224,-ds1,-pb2,st/2 } \\
& \text { k,225,-pd1,pt1,-st/2 } \\
& \text { k,226,-pd1,pb1,-st/2 } \\
& \text { k,227,-pd2,-pt2,-st/2 } \\
& \text { k,228,-pd2,-pb2,-st/2 } \\
& \text { k,229,-dsl,pt1,-st/2 } \\
& \text { k,230,-dsl,pb1,-st/2 } \\
& \text { k,231,-ds1,-pt2,-st/2 } \\
& \text { k,232,-dsl,-pb2,-st/2 } \\
& \text { k,233,-pla1,pb1,pwa1, } \\
& \text { k,234,-pla1,pt1,pwa1, } \\
& \text { k,235,-pla12,pb1,pwa12, } \\
& \text { k,236,-pla12,pt1,pwa12, } \\
& \text { k,237,-pla1,-pb2,pwa1, } \\
& \text { k,238,-pla1,-pt2,pwa1, } \\
& \text { k,239,-pla12,-pb2,pwa12, } \\
& \text { k,240,-pla12,-pt2,pwa12, }
\end{aligned}
$$

k,242,dsl,-dsb,-3*st/8

k,243,dsl,-dsb,st/8

$\mathrm{k}, 244, \mathrm{dsl},-\mathrm{dsb}, 3 * \mathrm{st} / 8$

$\mathrm{k}, 245, \mathrm{fd},-\mathrm{dsb},-3 * \mathrm{st} / 8$

$\mathrm{k}, 246, \mathrm{fd},-\mathrm{dsb}, \mathrm{st} / 8$

k,247,fd,-dsb, $3 *$ st $/ 8$

$\mathrm{k}, 248$,dsl,dst, $-3 *$ st $/ 8$

k,249,dsl,dst,st/8

$\mathrm{k}, 250, \mathrm{dsl}, \mathrm{dst}, 3 * \mathrm{st} / 8$

$\mathrm{k}, 251,-\mathrm{fd},-\mathrm{dsb},-3 * \mathrm{st} / 8$

k,252,-fd,-dsb,st/8

k,253,-fd,-dsb, $3 *$ st $/ 8$ 


$$
\begin{aligned}
& \text { k,254,-dsl,dst,-3*st/8 } \\
& \mathrm{k}, 255 \text {,-dsl,dst,st/8 } \\
& \mathrm{k}, 256,-\mathrm{ds} 1, \mathrm{dst}, 3 * \mathrm{st} / 8 \\
& \mathrm{k}, 257, \mathrm{fd}, \mathrm{dst},-3 * \mathrm{st} / 8 \\
& \mathrm{k}, 258 \text {,fd,dst,st/8 } \\
& \mathrm{k}, 259, \mathrm{fd}, \mathrm{dst}, 3 * \mathrm{st} / 8 \\
& \mathrm{k}, 260,-\mathrm{fd}, \mathrm{dst},-3 * \mathrm{st} / 8 \\
& \text { k,261,-fd,dst,st/8 } \\
& \mathrm{k}, 262,-\mathrm{fd}, \mathrm{dst}, 3 * \mathrm{st} / 8 \\
& \mathrm{k}, 263,-\mathrm{ds} 1,-\mathrm{dsb},-3 * \mathrm{st} / 8 \\
& \text { k,264,-dsl,-dsb,st/8 } \\
& \text { k,265,-ds1,-dsb,3*st/8 } \\
& \text { k,266,d,-bsn,-3*st/8 } \\
& \text { k,267,d,-bsn, st/8 } \\
& \text { k,268,d,-bsn, } 3 * \text { st } / 8 \\
& \text { k,269,-ds1,-dbf,-3*st/8, } \\
& \text { k,270,-dsl,-dbf,-st/8, } \\
& \text { k,271,-ds1,-dbf,st/8, } \\
& \text { k,272,-ds1,-dbf,3*st/8, } \\
& \mathrm{k}, 273,-\mathrm{fd},-\mathrm{dbf},-3 * \mathrm{st} / 8 \text {, } \\
& \text { k,274,-fd,-dbf,-st/8, } \\
& \mathrm{k}, 275,-\mathrm{fd},-\mathrm{dbf}, \mathrm{st} / 8 \text {, } \\
& \text { k,276,-fd,-dbf,3*st/8, } \\
& \text { k,277,dsl,-dbf,3*st/8, } \\
& \text { k,278,dsl,-dbf,st/8, } \\
& \text { k,279,dsl,-dbf,-st/8, } \\
& \text { k,280,dsl,-dbf,-3*st/8, } \\
& \text { k,281,fd,-dbf, } 3 * \text { st/8, } \\
& \mathrm{k}, 282, \mathrm{fd},-\mathrm{dbf}, \mathrm{st} / 8 \text {, } \\
& \text { k,283,fd,-dbf,-st/8, } \\
& \mathrm{k}, 284, \mathrm{fd},-\mathrm{dbf},-3 * \mathrm{st} / 8 \text {, } \\
& \mathrm{k}, 285,-\mathrm{ds} 1, \mathrm{dtf},-3 * \mathrm{st} / 8 \text {, } \\
& \text { k,286,-dsl,dtf,-st/8, } \\
& \mathrm{k}, 287 \text {,-dsl,dtf,st/8, } \\
& \text { k,288,-dsl,dtf, } 3 * \text { st/8, } \\
& \text { k,289,-fd,dtf,-3*st/8, } \\
& \text { k,290,-fd,dtf,-st/8, } \\
& \text { k,291,-fd,dtf,st/8, } \\
& \text { k,292,-fd,dtf, } 3 * \text { st/8, } \\
& \text { k,293,dsl,dtf,3*st/8, } \\
& \text { k,294,dsl,dtf,st/8, } \\
& \text { k,295,dsl,dtf,-st/8, } \\
& \text { k,296,dsl,dtf,-3*st/8, } \\
& \mathrm{k}, 297, \mathrm{fd}, \mathrm{dtf}, 3 * \mathrm{st} / 8 \text {, }
\end{aligned}
$$




$$
\begin{aligned}
& \text { k,298,fd,dtf,st/8, } \\
& \text { k,299,fd,dtf,-st/8, } \\
& \mathrm{k}, 300, \mathrm{fd}, \mathrm{dtf},-3 * \mathrm{st} / 8 \text {, } \\
& \mathrm{k}, 301, \mathrm{wt} / 2,-\mathrm{bsn}, 3 * \mathrm{st} / 8 \text {, } \\
& \text { k,302,wt/2,-bsn,st/8, } \\
& \text { k,303,wt/2,-bsn,-st/8, } \\
& \text { k,304,wt/2,-bsn,-3*st/8, } \\
& \mathrm{k}, 305,-\mathrm{wt} / 2,-\mathrm{bsn}, 3{ }^{*} \mathrm{st} / 8 \text {, } \\
& \text { k,306,-wt/2,-bsn,st/8, } \\
& \text { k,307,-wt/2,-bsn,-st/8, } \\
& \text { k,308,-wt/2,-bsn,-3*st/8, } \\
& \mathrm{k}, 309 \text {,wt/2,tsn, } 3 * \text { st/8, } \\
& \mathrm{k}, 310, \mathrm{wt} / 2, \mathrm{tsn}, \mathrm{st} / 8 \text {, } \\
& \text { k,311,wt/2,tsn,-st/8, } \\
& \mathrm{k}, 312, \mathrm{wt} / 2, \mathrm{tsn},-3 * \mathrm{st} / 8 \text {, } \\
& \mathrm{k}, 313 \text {,-wt/2,tsn, } 3 * \text { st } / 8 \text {, } \\
& \mathrm{k}, 314,-\mathrm{wt} / 2, \mathrm{tsn}, \mathrm{st} / 8 \text {, } \\
& \mathrm{k}, 315,-\mathrm{wt} / 2, \mathrm{tsn},-\mathrm{st} / 8 \text {, } \\
& \text { k,316,-wt/2,tsn,-3*st/8, } \\
& \text { k,317,-d,-bsn,-3*st/8 } \\
& \text { k,318,-d,-bsn, st/8 } \\
& \text { k,319,-d,-bsn, } 3 * \text { st } / 8 \\
& \mathrm{k}, 320, \mathrm{~d}, \mathrm{tsn},-3 * \mathrm{st} / 8 \\
& \mathrm{k}, 321, \mathrm{~d}, \mathrm{tsn}, \mathrm{st} / 8 \\
& \text { k,322,d,tsn, } 3 * \text { st } / 8 \\
& \mathrm{k}, 323,-\mathrm{d}, \mathrm{tsn},-3 * \mathrm{st} / 8 \\
& \mathrm{k}, 324,-\mathrm{d}, \mathrm{tsn}, \mathrm{st} / 8 \\
& \mathrm{k}, 325,-\mathrm{d}, \mathrm{tsn}, 3 * \mathrm{st} / 8 \\
& \text { k,326,-ds1,dst,-st/2 } \\
& \text { k,327,-dsl,dst,st/2 } \\
& \text { k,328,-fd,dst,-st/2 } \\
& \mathrm{k}, 329,-\mathrm{fd}, \mathrm{dst}, \mathrm{st} / 2 \\
& \text { k,330,dsl,dst,-st/2 } \\
& \mathrm{k}, 331, \mathrm{dsl}, \mathrm{dst}, \mathrm{st} / 2 \\
& \mathrm{k}, 332, \mathrm{fd}, \mathrm{dst},-\mathrm{st} / 2 \\
& \text { k,333,fd,dst,st/2 } \\
& \text { k,334,-e1x,ctop1,e1z, } \\
& \text { k,335,-e1x,cang1,e1z, } \\
& \text { k,336,-e1x,cbot1,e1z, } \\
& \text { k,337,-cl1x,pt1,cl1z, } \\
& \text { k,338,-e1x,pb1,e1z, } \\
& \text { k,339,-e1x,pt1,e1z, }
\end{aligned}
$$




$$
\begin{aligned}
& \text { k,340,-cl1x,ctop1,cl1z, } \\
& \text { k,341,-cl1x,cang1,cl1z, } \\
& \text { k,342,-cl1x,cbot1,cl1z, } \\
& \text { k,343,-cl11x,ctop1,cl11z, } \\
& \text { k,344,-cl11x,cang1,cl11z, } \\
& \text { k,345,-cl11x,cbot1,cl11z, } \\
& \text { k,346,-cl111x,ctop1,cl111z, } \\
& \text { k,347,-cl111x,cang1,cl111z, } \\
& \text { k,348,-pla1,ctop1,pwa1, } \\
& \text { k,349,-pla1,cang1,pwa1, } \\
& \text { k,350,-pla1,cbot1,pwa1, } \\
& \text { k,351,-cl22x,ctop1,cl22z, } \\
& \text { k,352,-cl22x,cang1,cl22z, } \\
& \text { k,353,-cl22x,cbot1,cl22z, } \\
& \text { k,354,-c1222x,ctop1,cl222z, } \\
& \text { k,355,-cl222x,cang1,cl222z, } \\
& \text { k,357,-cl3x,ctop1,cl3z } \\
& \text { k,358,-cl3x,cang1,cl3z } \\
& \text { k,359,-cl3x,cbot1,cl3z } \\
& \text { k,360,-cl33x,ctop1,cl33z } \\
& \text { k,361,-cl33x,cang1,cl33z } \\
& \text { k,362,-cl33x,cbot1,cl33z } \\
& \text { k,363,-cl333x,ctop1,cl333z } \\
& \text { k,364,-cl333x,cang1,cl333z } \\
& \text { k,365,-cl1x,pb1,cl1z, } \\
& \text { k,366,-pla12,ctop1,pwa12, } \\
& \text { k,367,-pla12,cang1,pwa12, } \\
& \text { k,368,-pla12,cbot1,pwa12, } \\
& \text { k,369,-e1x,-ctop2,e1z } \\
& \text { k,370,-e1x,-cang2,e1z } \\
& \text { k,371,-e1x,-cbot2,e1z } \\
& \text { k,372,-e1x,-pt2,e1z, } \\
& \text { k,373,-e1x,-pb2,e1z, } \\
& \text { k,374,-cl1x,-pt2,cl1z, } \\
& \text { k,375,-cl1x,-ctop2,cl1z, } \\
& \text { k,376,-cl1x,-cang2,cl1z, } \\
& \text { k,377,-cl1x,-cbot2,cl1z, } \\
& \text { k,378,-cl11x,-ctop2,cl11z, } \\
& \text { k,379,-cl11x,-cang2,cl11z, } \\
& \text { k,380,-cl11x,-cbot2,cl11z, } \\
& \text { k,383,-pla1,-cang2,pwa1, } \\
& \text { k,382,-cl111x,-cang2,cl111z, }
\end{aligned}
$$




$$
\begin{aligned}
& \text { k,386,-cl22x,-ctop2,cl22z, } \\
& \text { k,387,-cl22x,-cang2,cl22z, } \\
& \text { k,388,-cl22x,-cbot2,cl22z, } \\
& \text { k,389,-cl222x,-ctop2,cl222z, } \\
& \text { k,390,-cl222x,-cang2,cl222z, } \\
& \text { k,392,-cl3x,-ctop2,cl3z } \\
& \text { k,393,-cl3x,-cang2,cl3z } \\
& \text { k,394,-cl3x,-cbot2,cl3z } \\
& \text { k,395,-cl33x,-ctop2,cl33z } \\
& \text { k,396,-cl33x,-cang2,cl33z } \\
& \text { k,397,-cl33x,-cbot2,cl33z } \\
& \text { k,398,-c1333x,-ctop2,cl333z } \\
& \text { k,399,-cl333x,-cang2,cl333z } \\
& \text { k,400,-cl1x,-pb2,cl1z, } \\
& \text { k,401,-pla12,-ctop2,pwa12, } \\
& \text { k,402,-pla12,-cang2,pwa12, } \\
& \text { k,403,-pla12,-cbot2,pwa12, }
\end{aligned}
$$
a, $1,2,11,12$,
a, 1,2,30,29,
a, $1,12,40,29$,
a, 11,12,40,39,
a, $2,11,39,30$,
a,29,40,39,30,
va, $1,2,3,4,5,6$,
a, $2,3,10,11$,
a, $2,3,31,30$,
a, 11,10,38,39,
a, $3,10,38,31$,
a,30,31,38,39,

va, $7,8,9,10,11,5$,

a, $3,4,9,10$,

a, 3,4,32,31,

a, $10,9,37,38$,

a, 4,9,37,32,

a,31,32,37,38,

va, $12,13,14,15,16,10$, 

a, $4,5,33,32$,
a, $4,5,8,9$,
a, $8,9,37,36$,
a, 5,8,36,33,
a,32,33,36,37,
va, 17,18,19,20,21,15,
a, $5,6,7,8$,
a, $6,7,35,34$,
a, 5,6,34,33,
a, 7,8,36,35,
a,33,34,35,36,
va, $22,23,24,25,26,20$,
a, 29,40,68,57,
a,29,30,58,57,
a, 40,39,67,68,
a,30,39,67,58,
a, 57,58,67,68,
va,27,28,29,30,31,6,
a,30,31,59,58,
a,39,38,66,67,
a,31,38,66,59,
a,58,59,66,67,
va,32,33,34,35,30,11,
a,31,32,60,59,
a,38,37,65,66,
a,32,60,65,37,
a, 59,60,65,66,
va,36,37,38,39,34,16,
a,32,33,61,60,
a,37,36,64,65,
a,33,36,64,61,
a, 60,61,64,65,
va,40,41,42,43,38,21, 


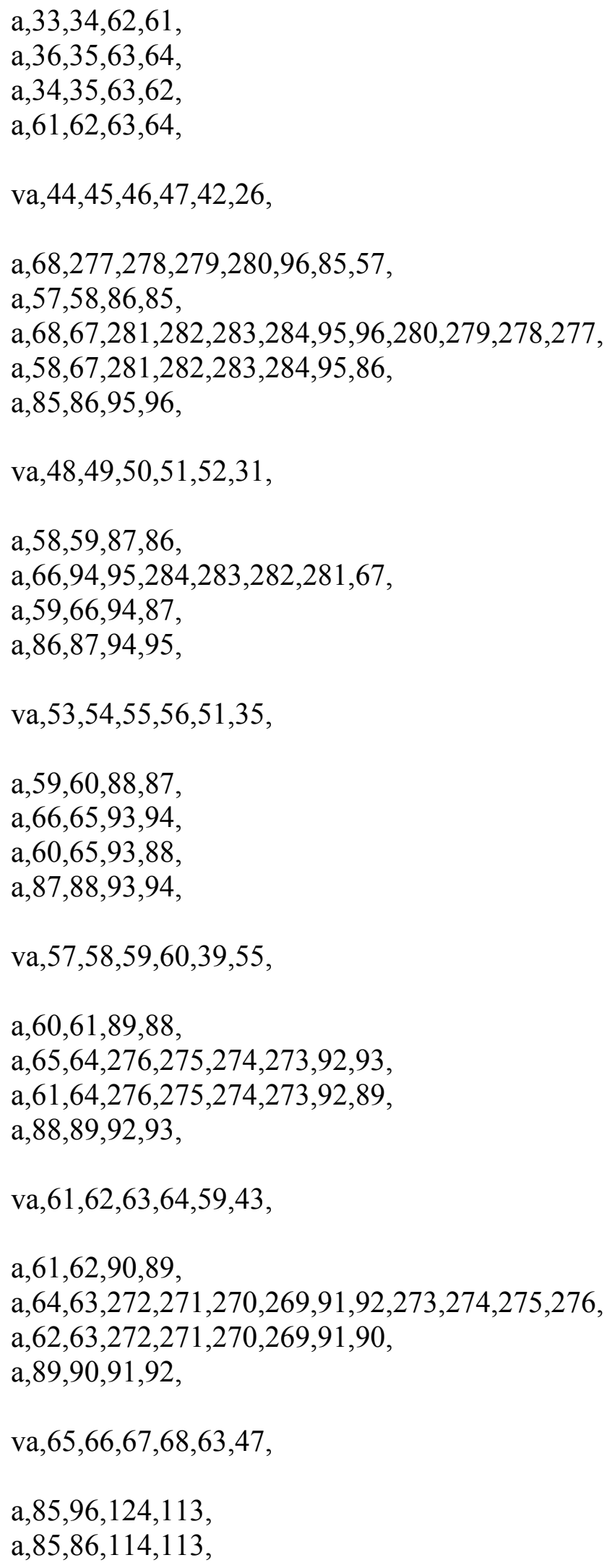




$$
\begin{aligned}
& \text { a,96,95,123,124, } \\
& \text { a, 86,95,123,114, } \\
& \text { a,113,114,123,124, } \\
& \text { va, } 69,70,71,72,73,52 \text {, } \\
& \text { a, } 86,87,115,114 \text {, } \\
& \text { a,95,94,122,123, } \\
& \text { a,87,94,122,115, } \\
& \text { a,114,115,122,123, } \\
& \text { va, } 74,75,76,77,72,56 \text {, } \\
& \text { a,87,88,116,115, } \\
& \text { a,94,93,121,122, } \\
& \text { a, } 88,93,121,116 \text {, } \\
& \text { a,115,116,121,122, } \\
& \text { va, } 78,79,80,81,76,60 \text {, } \\
& \text { a, 88,89,117,116, } \\
& \text { a,93,92,120,121, } \\
& \text { a,89,92,120,117, } \\
& \text { a,116,117,120,121, } \\
& \text { va, } 82,83,84,85,80,64 \text {, } \\
& \text { a,89,90,118,117, } \\
& \text { a,92,91,119,120, } \\
& \text { a,90,91,119,118, } \\
& \text { a,117,118,119,120, } \\
& \text { va, } 86,87,88,89,84,68 \text {, } \\
& \text { a,113,124,152,141, } \\
& \text { a,113,114,142,141, } \\
& \text { a, 124,123,151,152, } \\
& \text { a, 114,123,151,142, } \\
& \text { a,141,142,151,152, } \\
& \text { va,90,91,92,93,94,73, } \\
& \text { a,114,115,143,142, } \\
& \text { a, 123,122,150,151, } \\
& \text { a, 115,122,150,143, }
\end{aligned}
$$




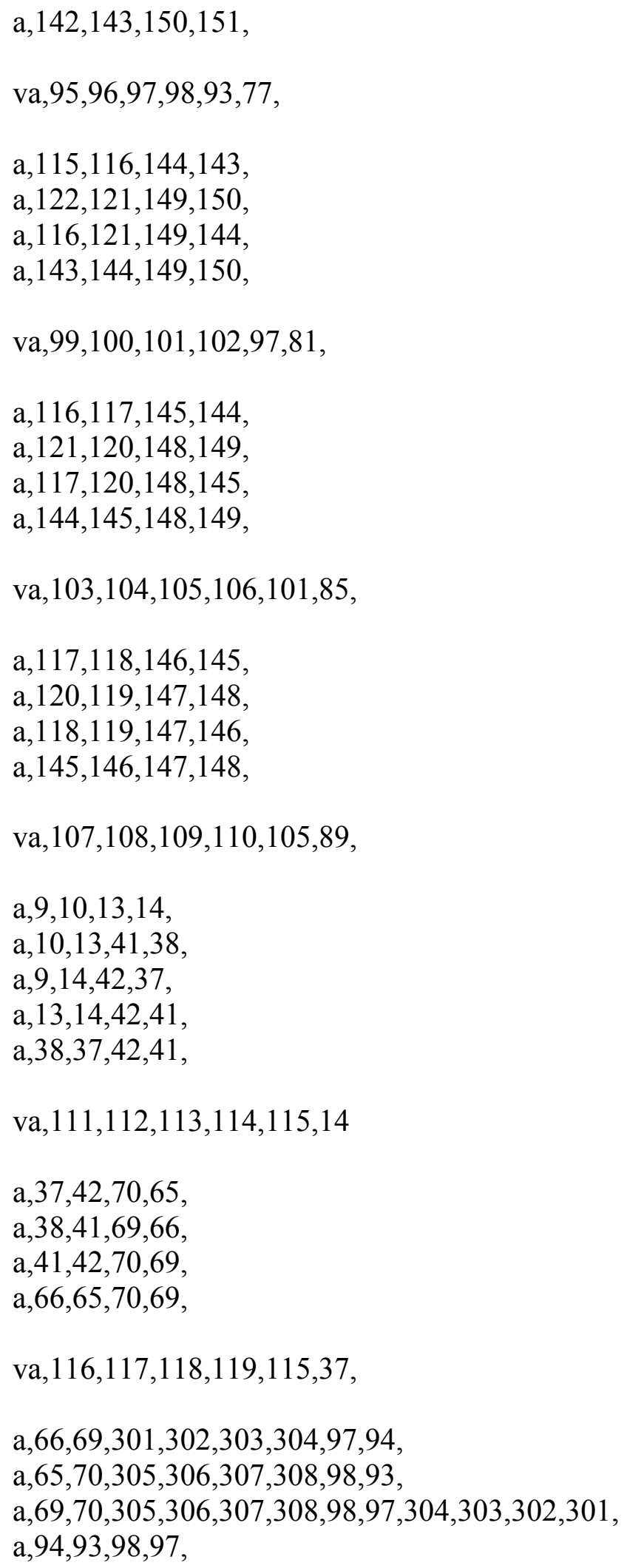




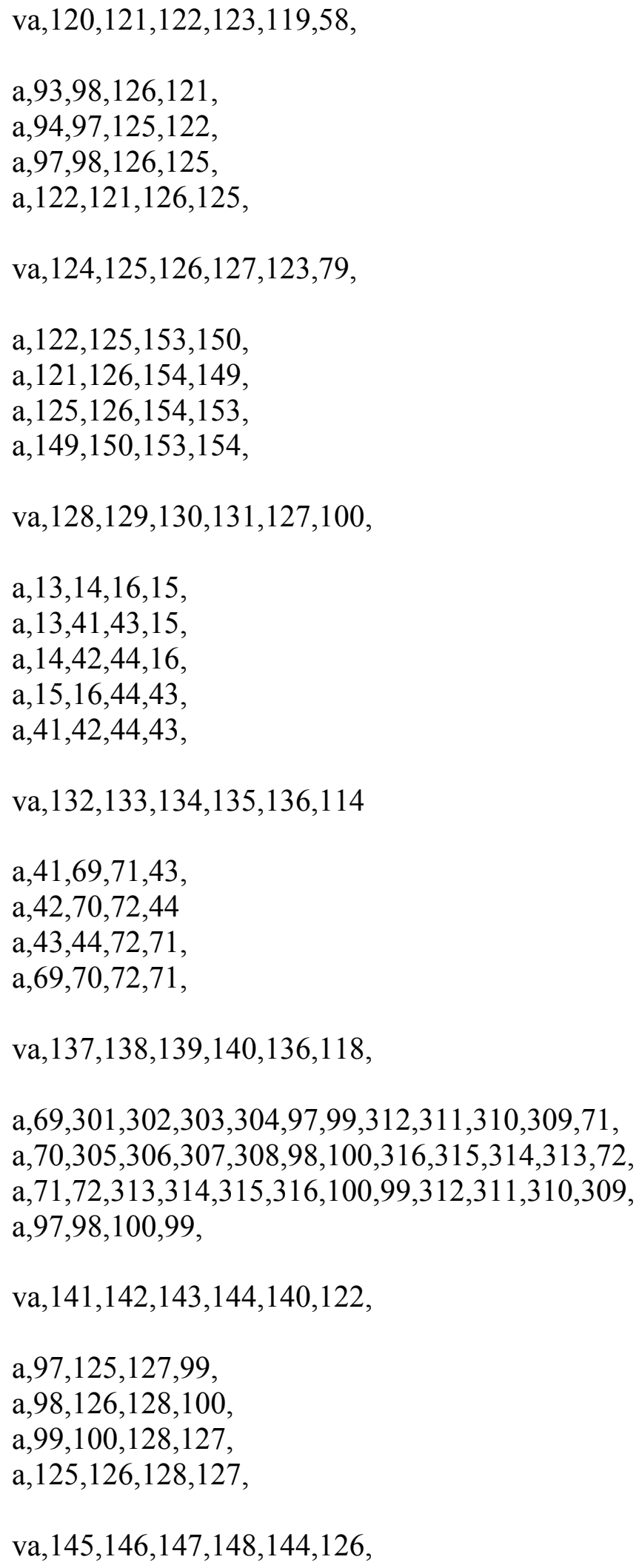

va, 141,142,143,144,140,122,

a,97,125,127,99, a, $98,126,128,100$, a,99,100,128,127, a, 125,126,128,127, va, $145,146,147,148,144,126$, 


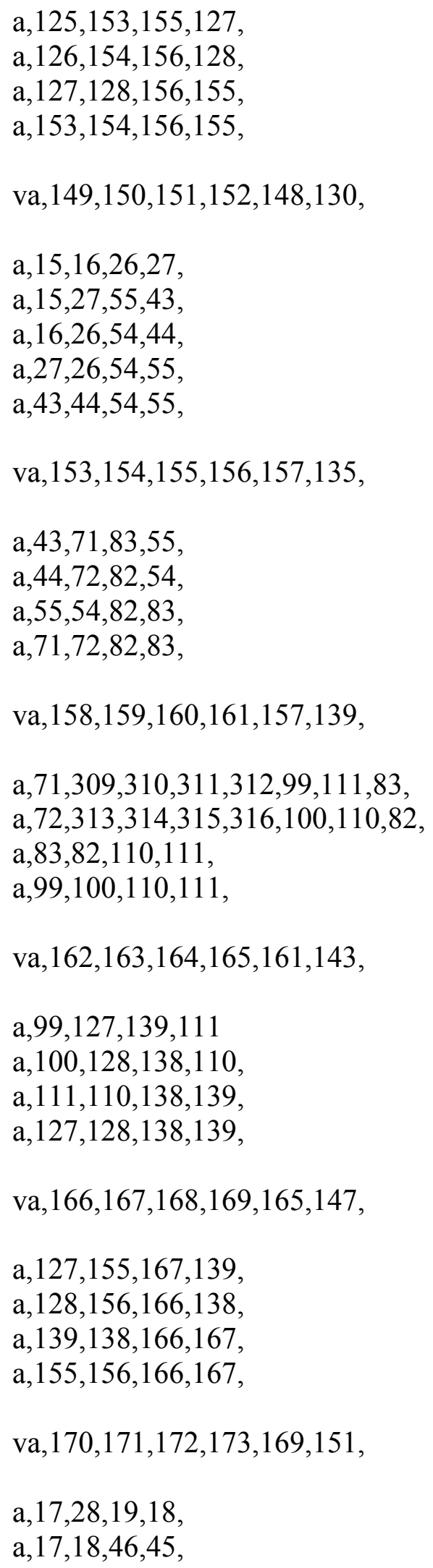




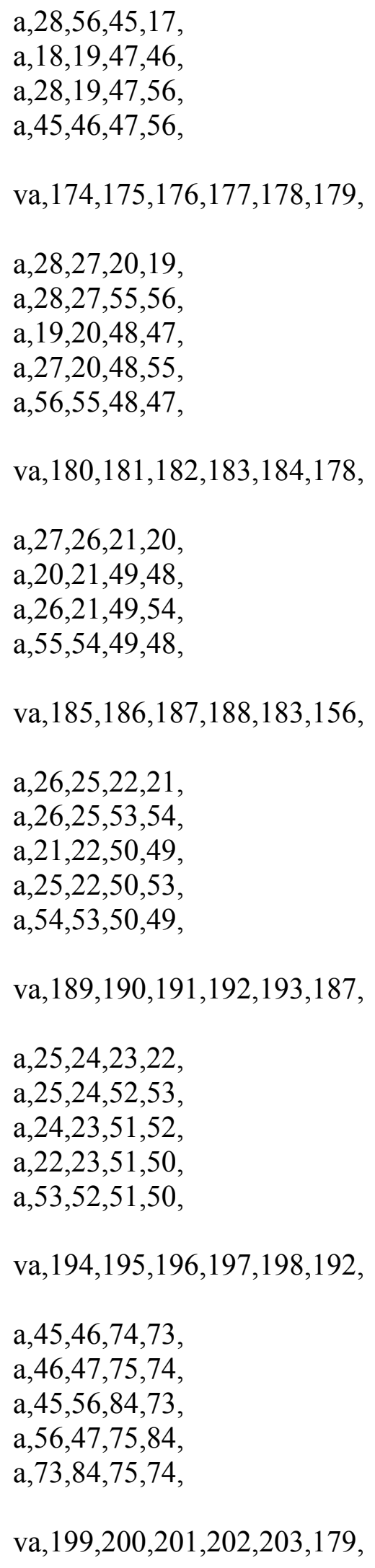




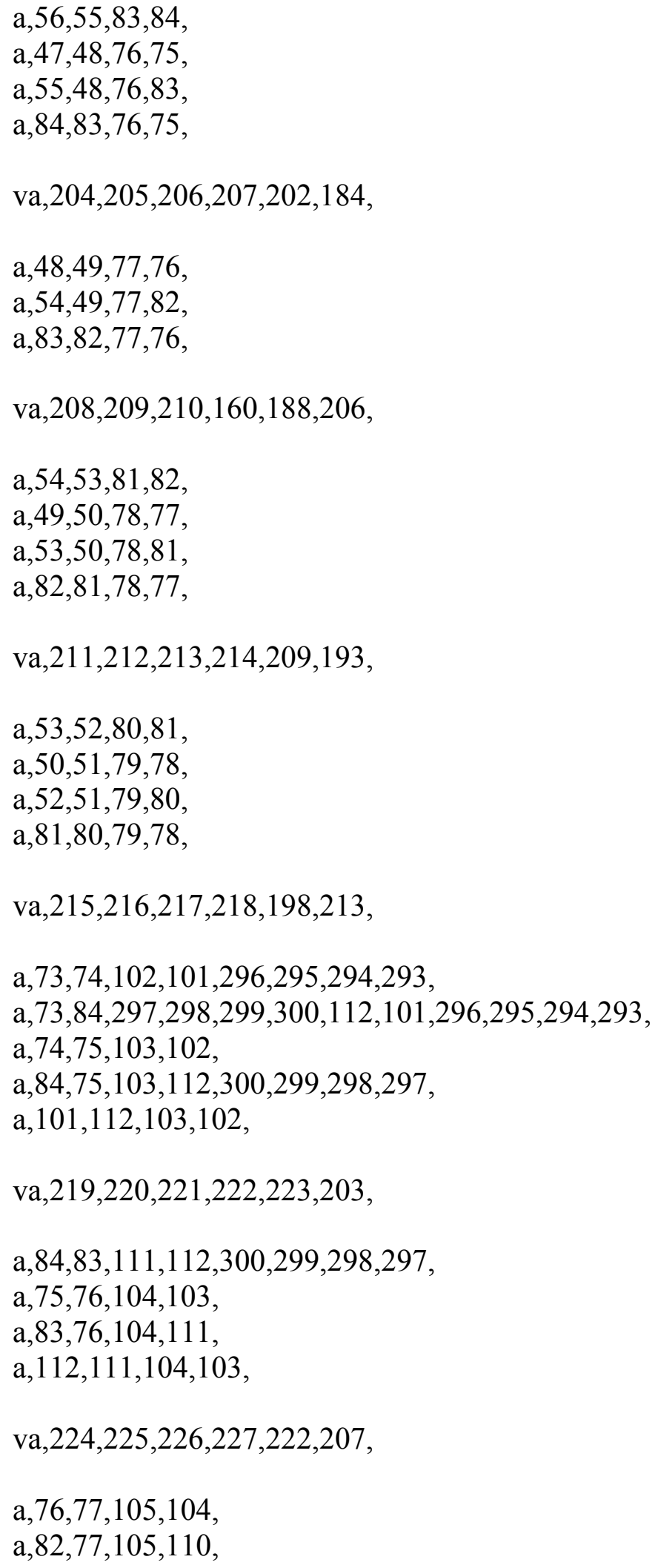




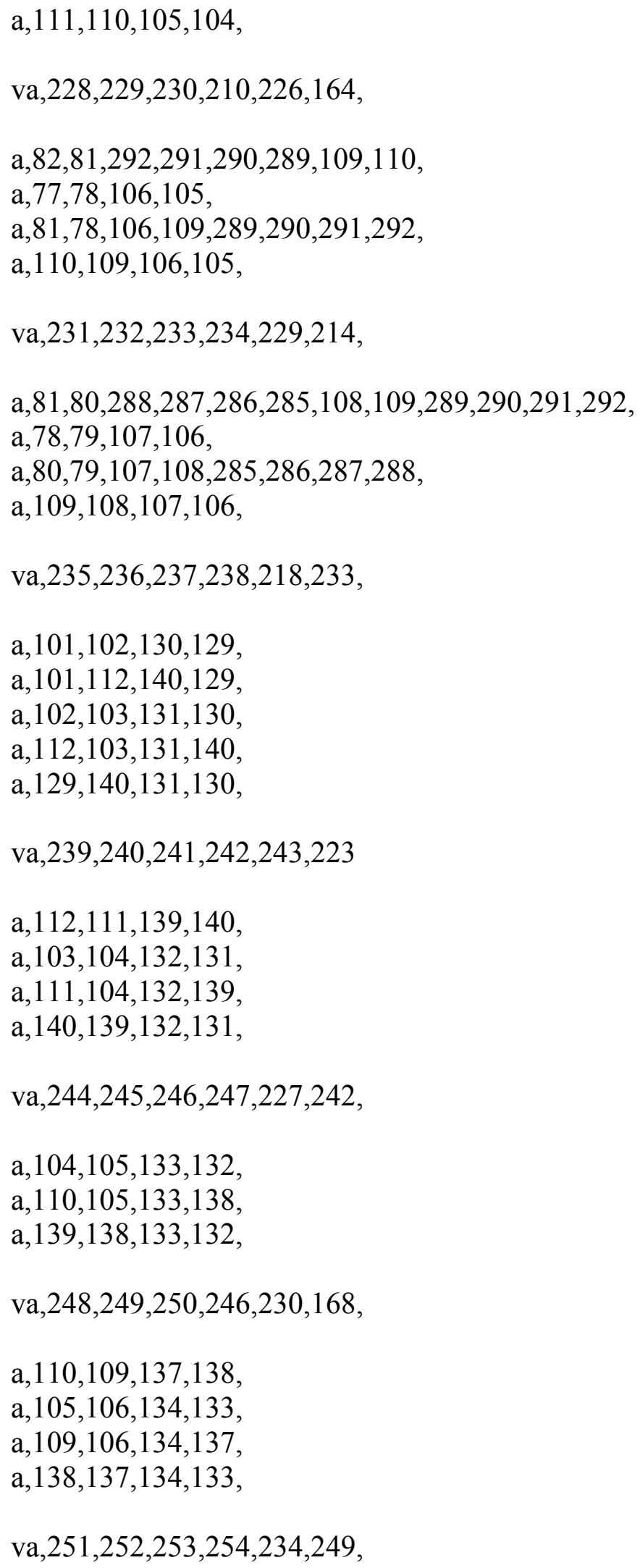




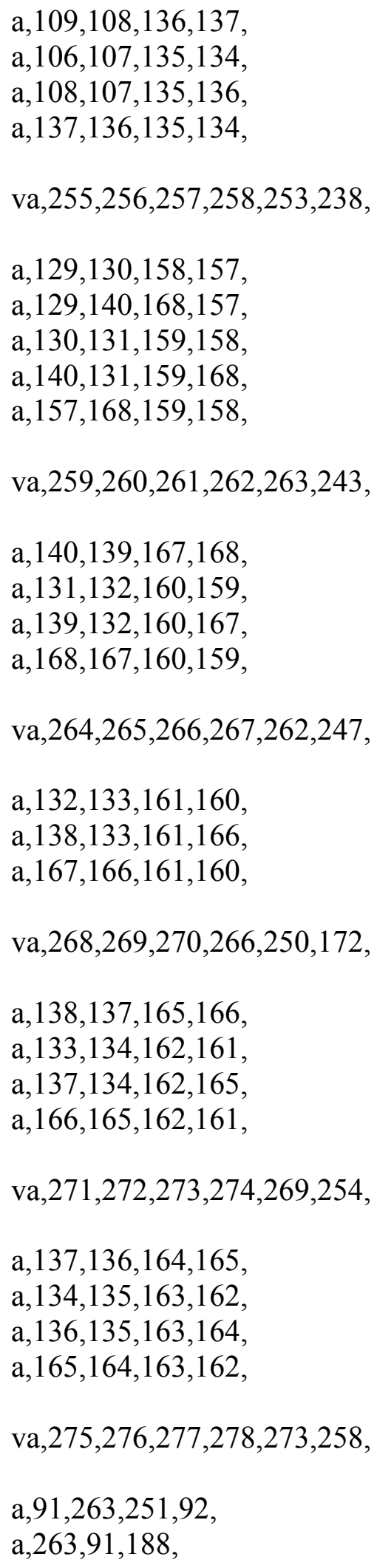




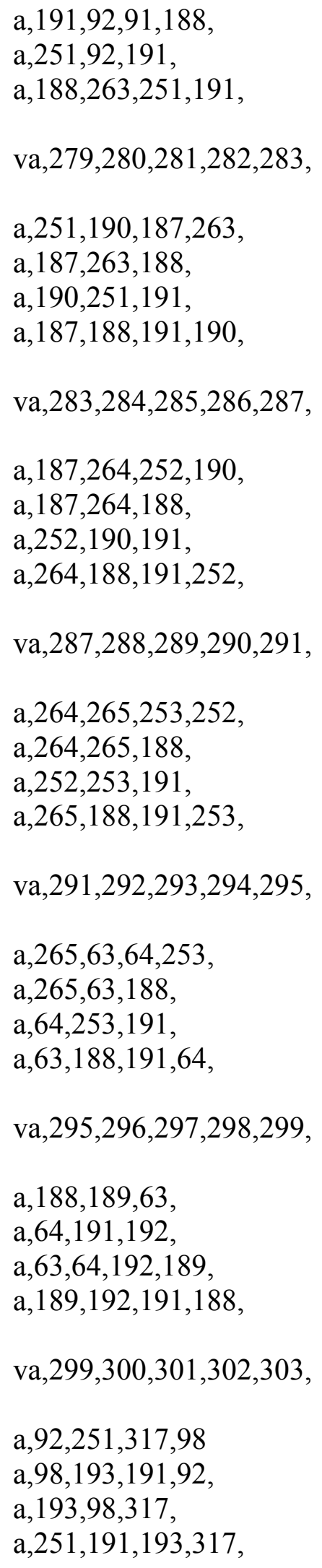




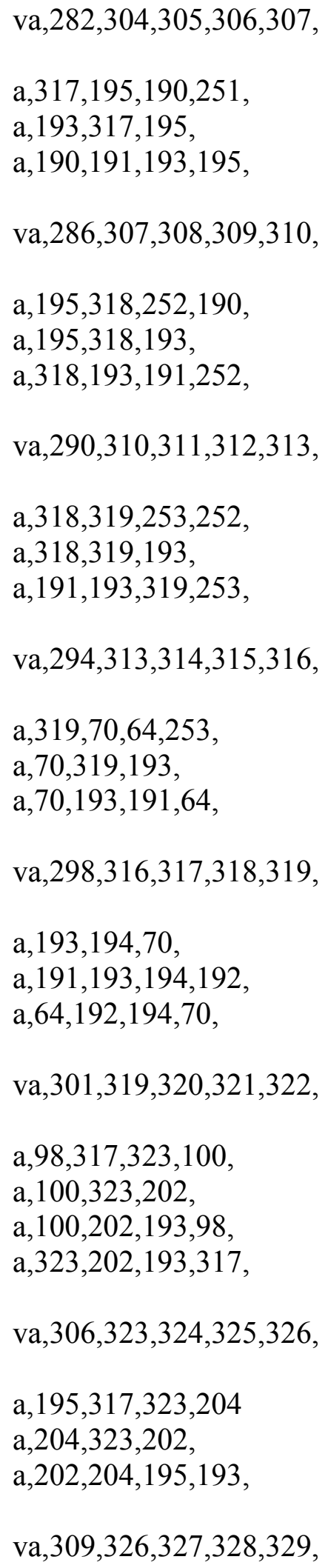




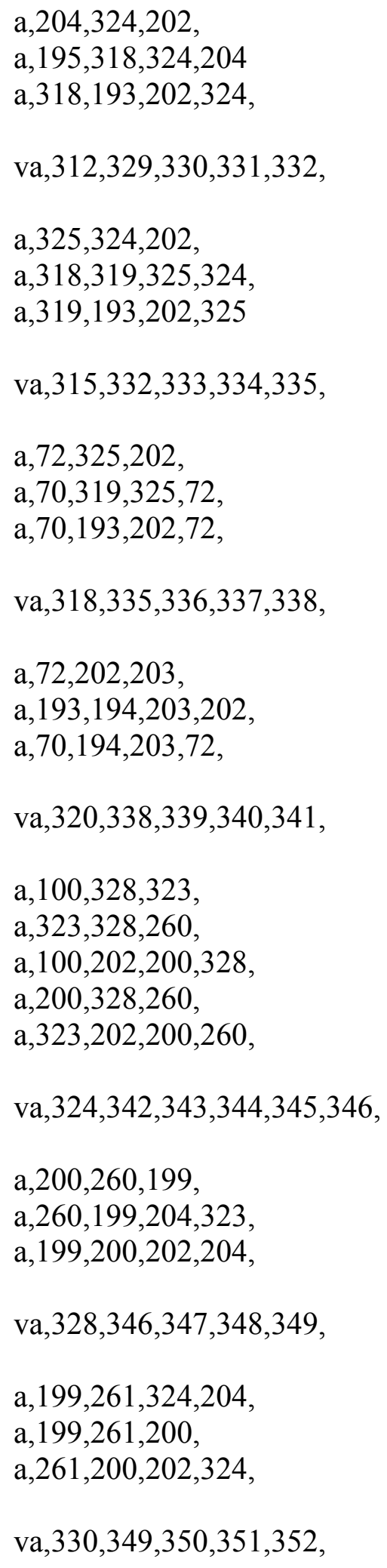




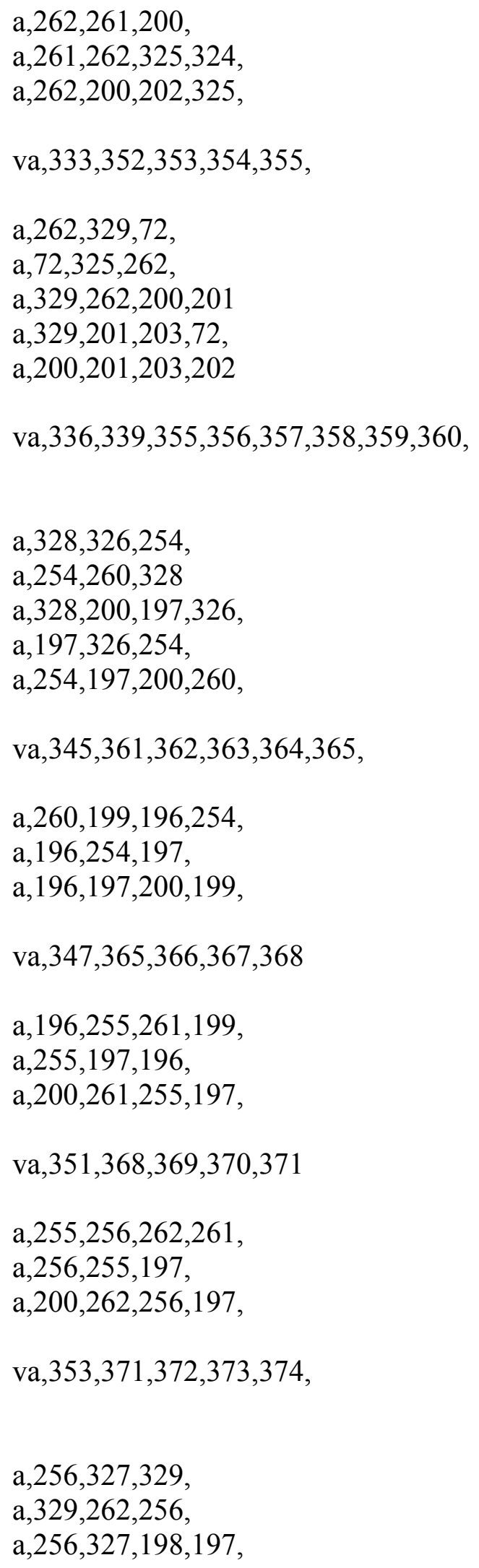




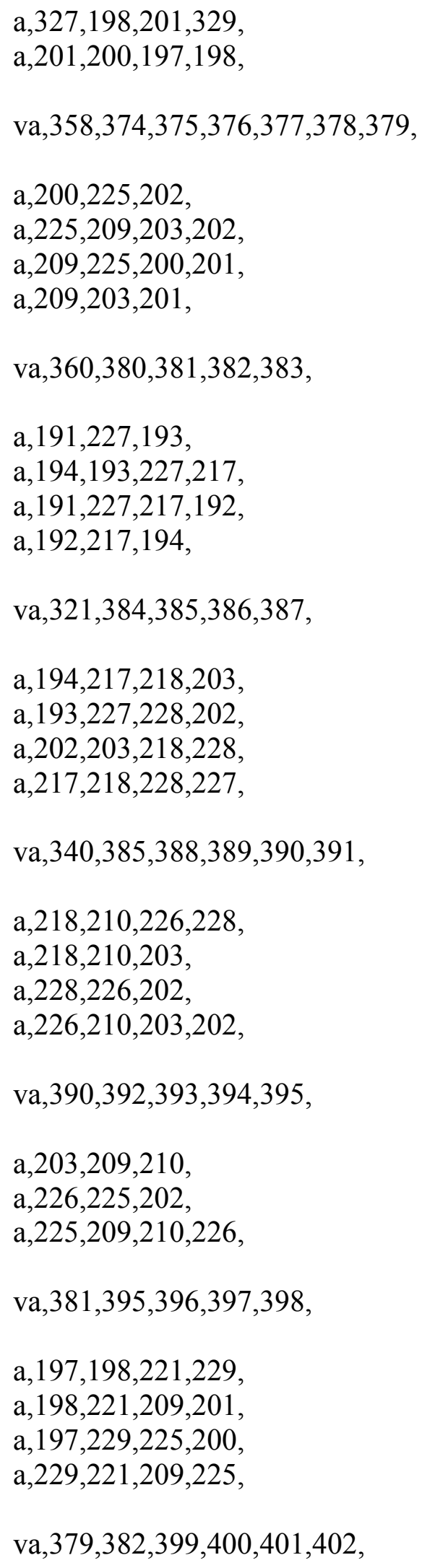




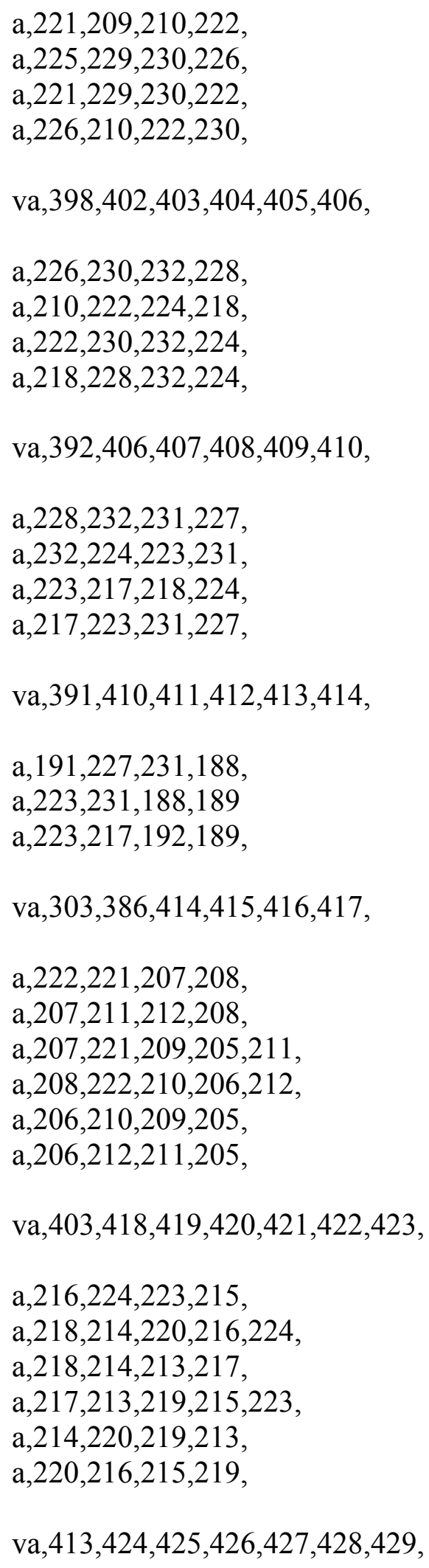




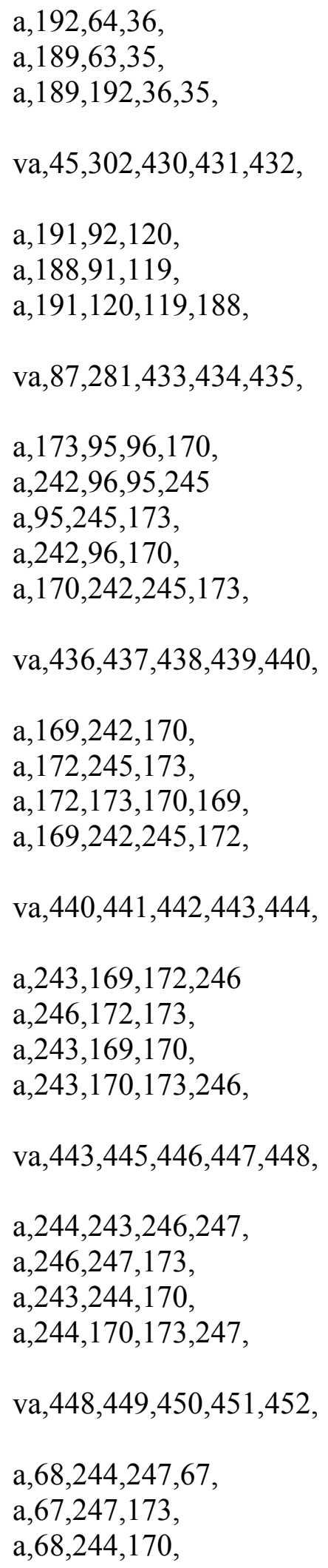




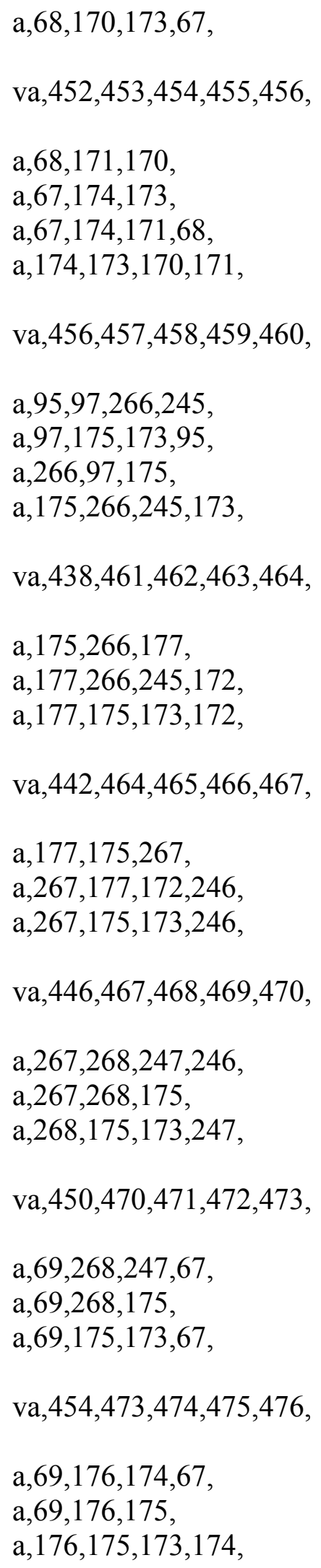




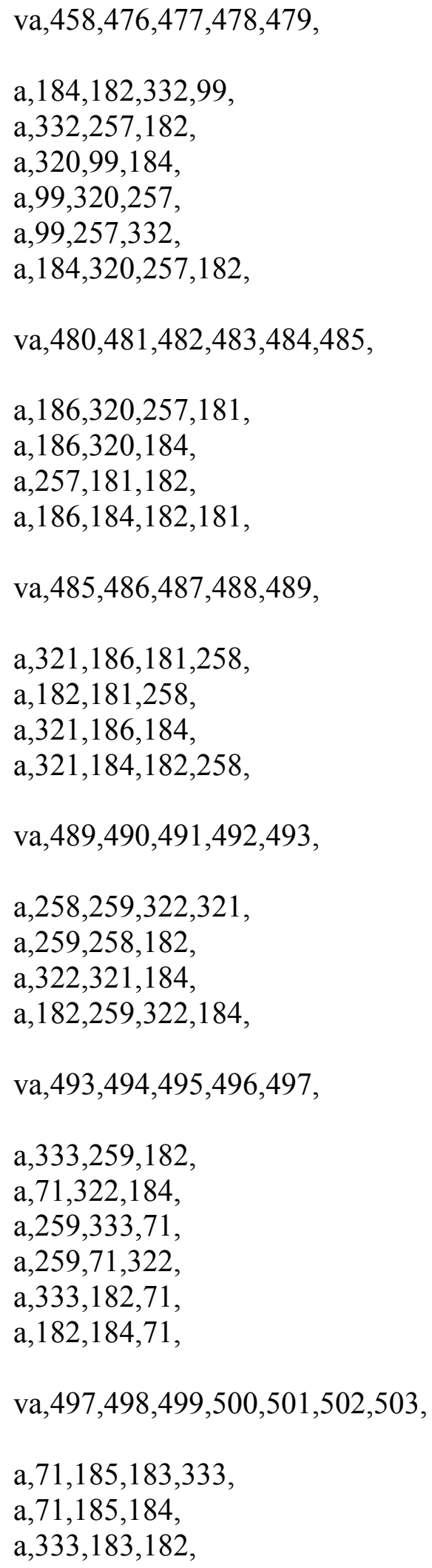




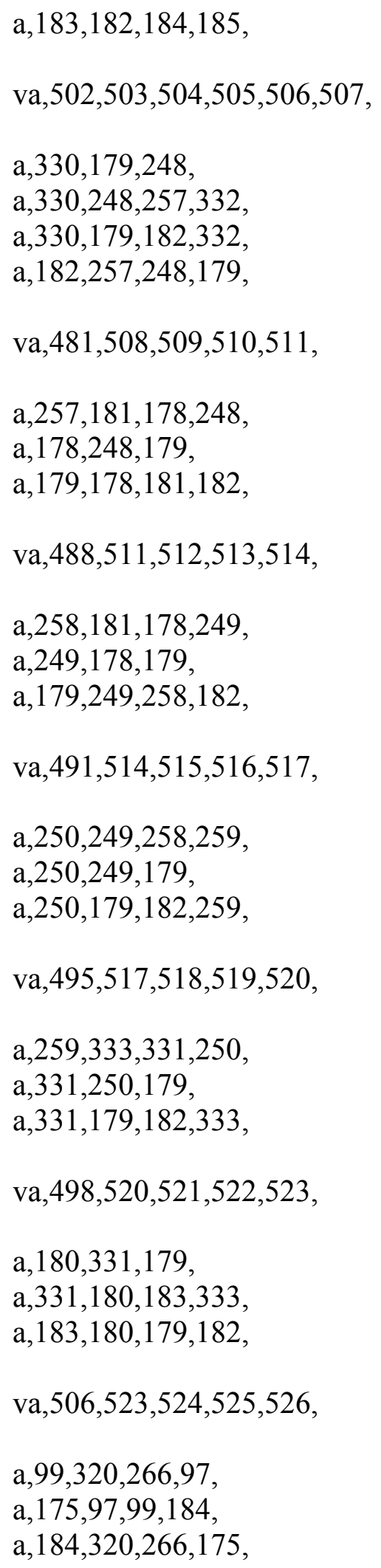


va, $482,463,527,528,529$,

a, 177,266,320,186,

a, 177,175,184,186,

va,487,465,529,530,531,

a,267,177,186,321,

a, 175,267,321,184,

va,492,468,531,532,533,

a,268,267,321,322,

a, 268, 175, 184,322,

va,496,472,533,534,535,

a, $69,268,322,71$

a, 69, 175, 184,71,

va,499,475,535,536,537,

a, 69, 176,185,71,

a, $176,175,184,185$

va,505,478,537,538,539,

a,39, 174,67,

a, $40,68,171$,

a,40,171,174,39,

va, $29,459,540,541,542$,

a, 173,123,95,

a, 124,96,170,

a, 170,124,123,173,

va,71,436,543,544,545,

a, 184,99,127,

a, $175,97,125$,

a, 125,175,184,127,

va, $145,528,546,547,548$, 


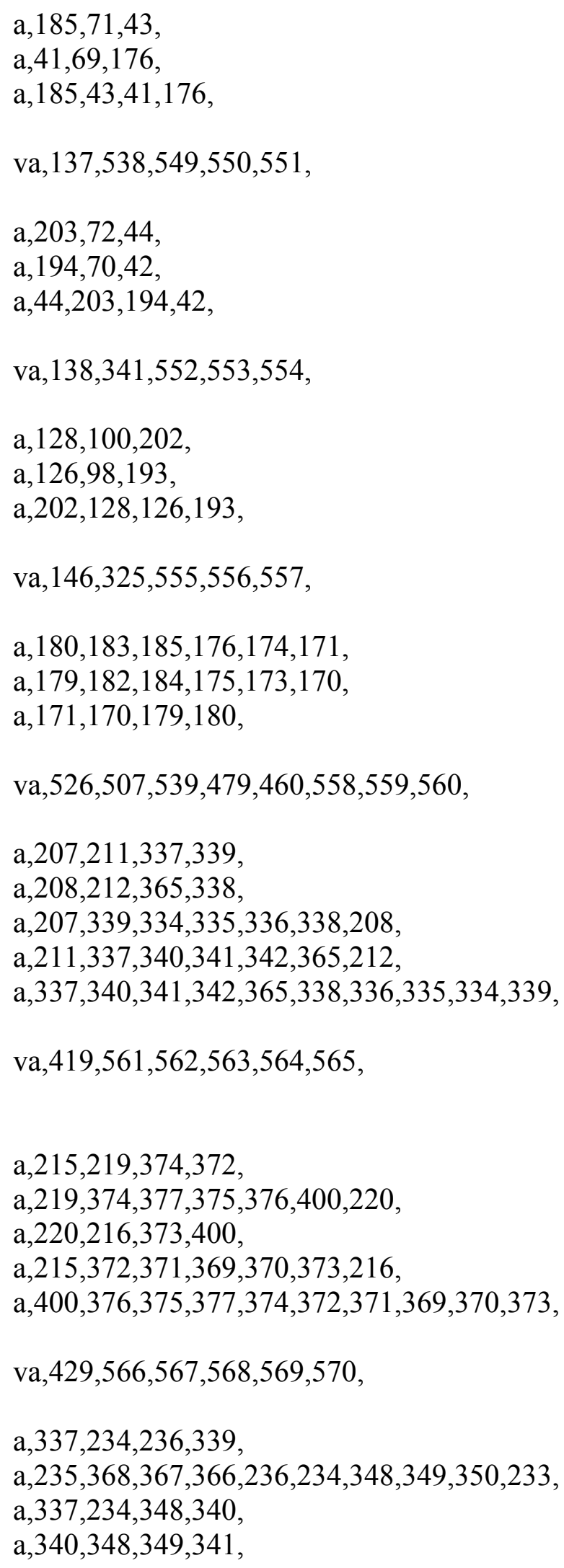




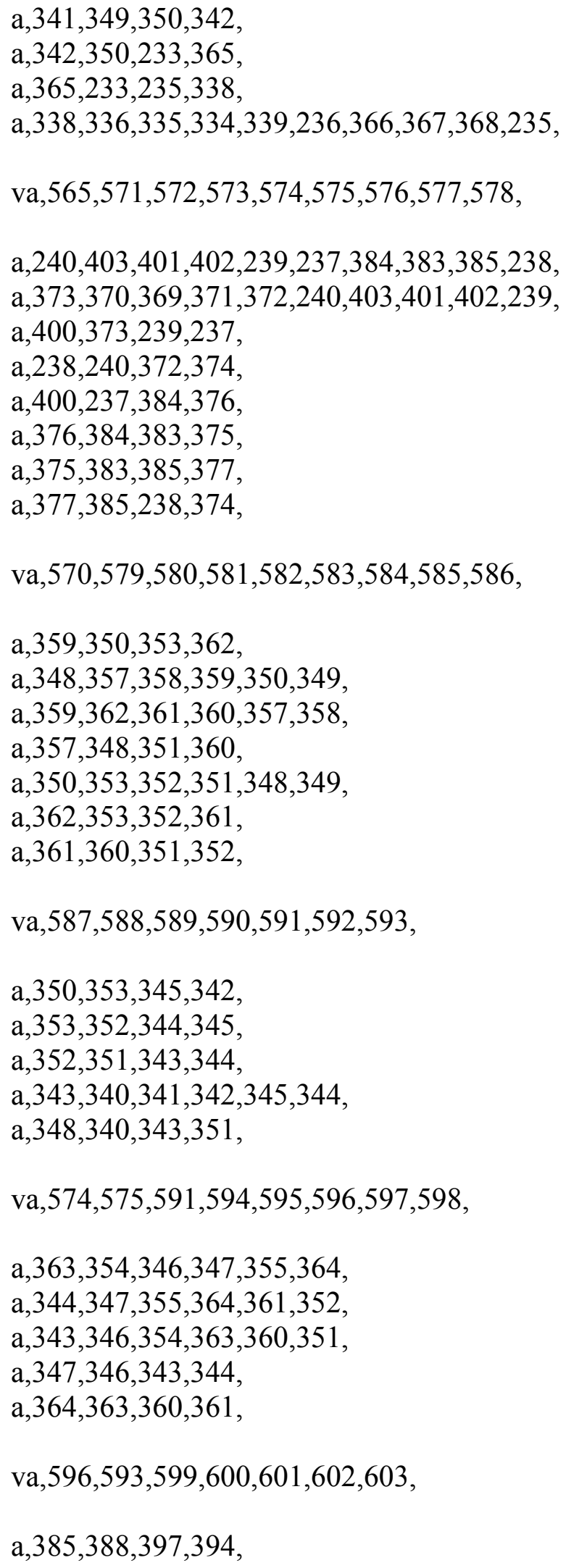




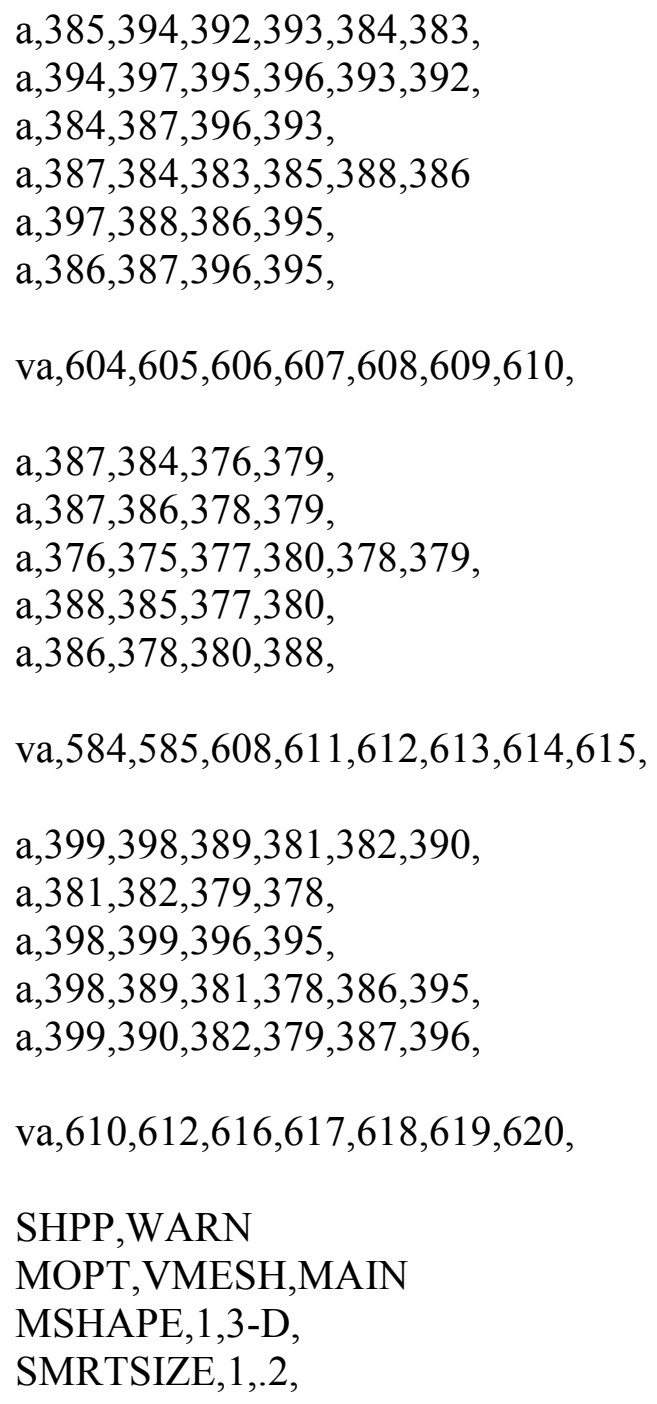




\begin{abstract}
NSEL,A,NODE,9443,9486,1,, NSEL,A,NODE,,13004,13006,1, NSEL,A,NODE,,13031,13033,1, NSEL,A,NODE,13060,13062,1, NSEL,A,NODE, 13128,13206,1, NSEL,A,NODE, 13270,13313,1, NSEL,A,NODE,13325,13368,1, NSEL,A,NODE,,13382,13407,1, NSEL,A,NODE, 13423,13470,1, NSEL,A,NODE,232914,232955,1, NSEL,A,NODE,,233240,233378,1, NSEL,A,NODE,,256933,257535,602,, NSEL,A,NODE,,257728,257826,1, NSEL,A,NODE, 258178,258233,1, NSEL,A,NODE,258384,258454,1, NSEL,A,NODE,,258666,258715,1, NSEL,A,NODE,,258989,259090,1,, CPINTF,UY,0.002
\end{abstract}

NSEL,ALL,

Boundary Conditions available on request. 


\subsection{MATLAB Code for Interpolation}

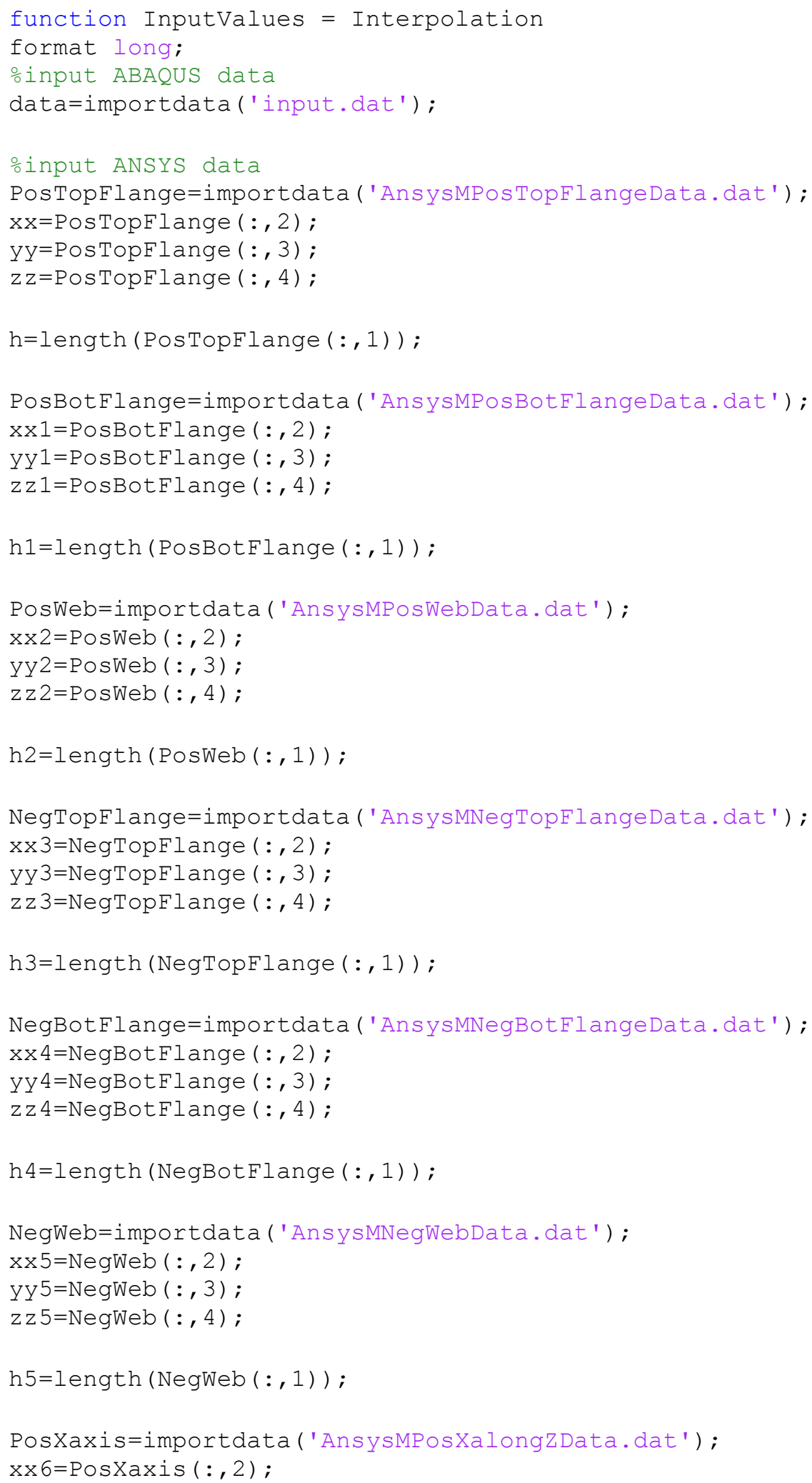




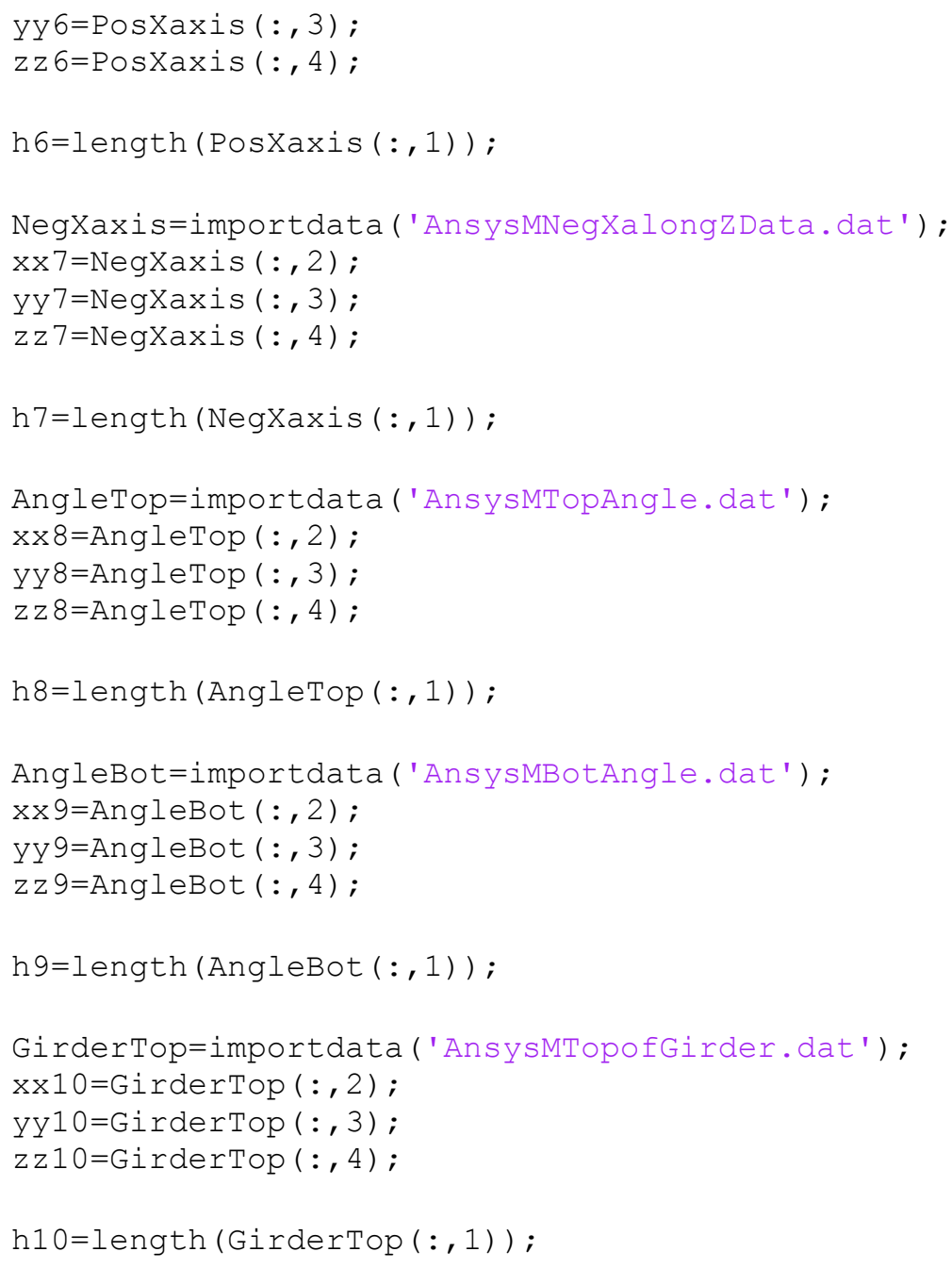




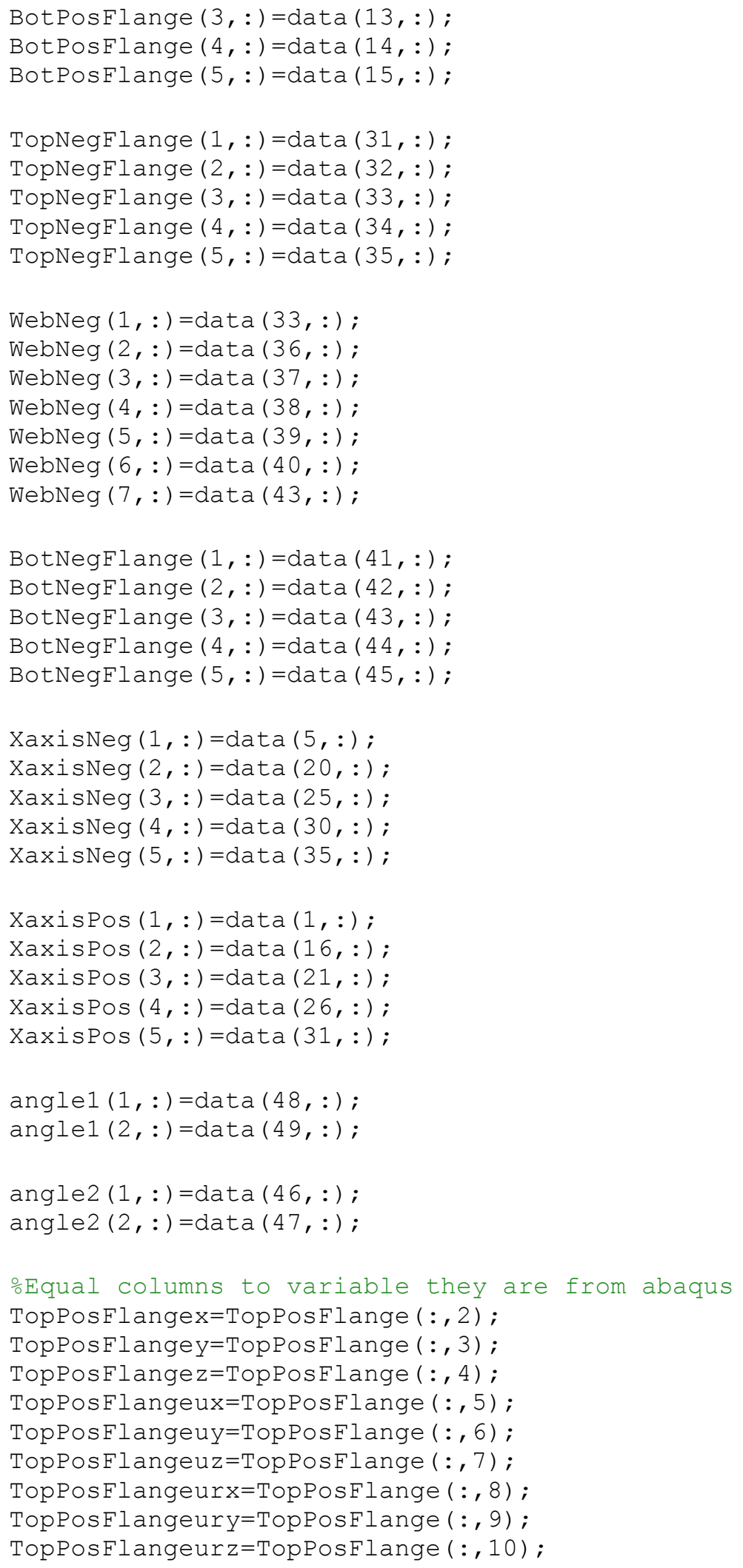




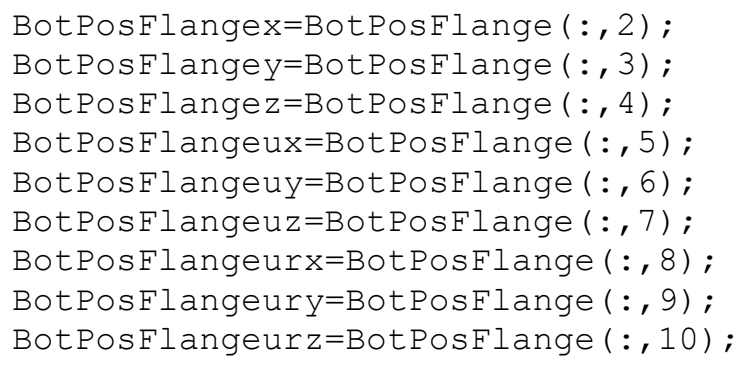




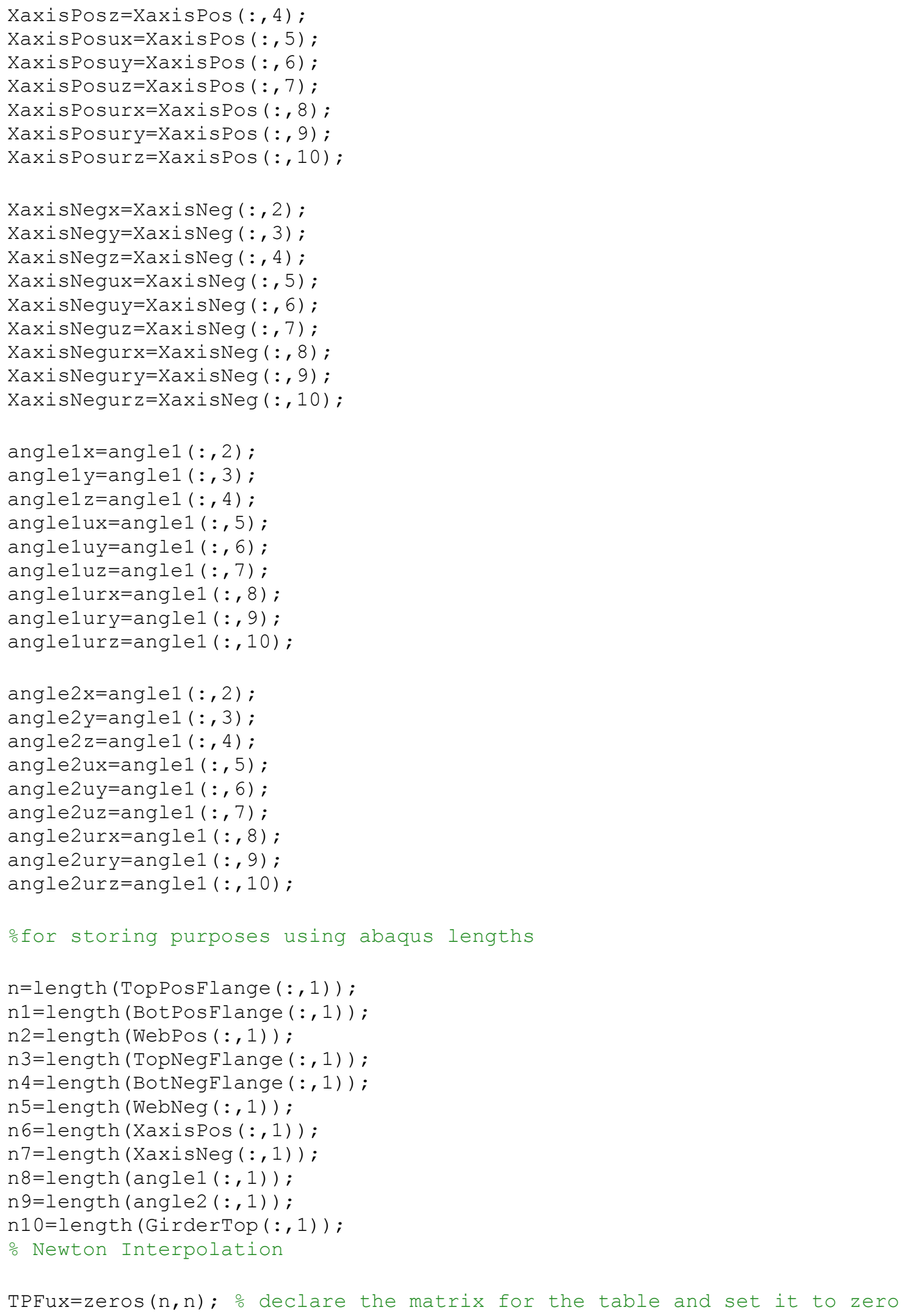




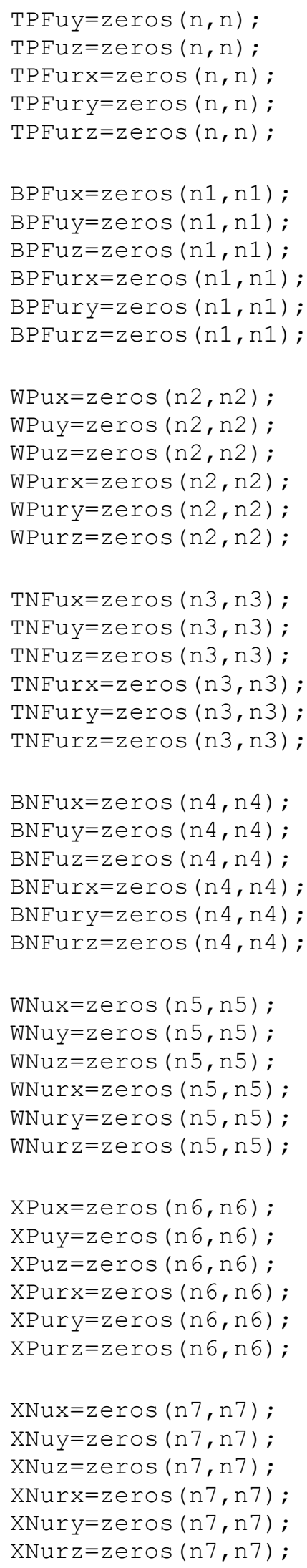




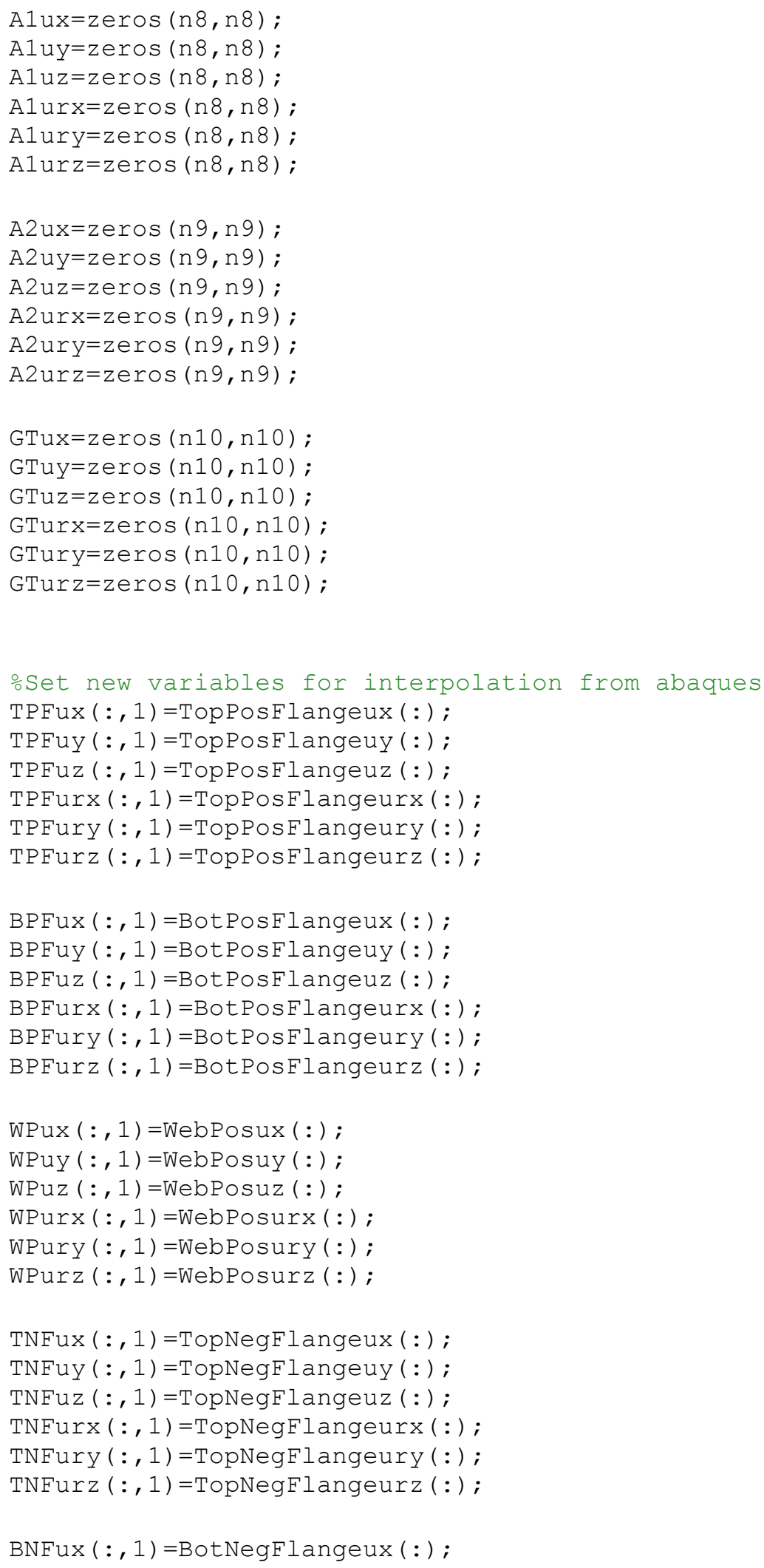




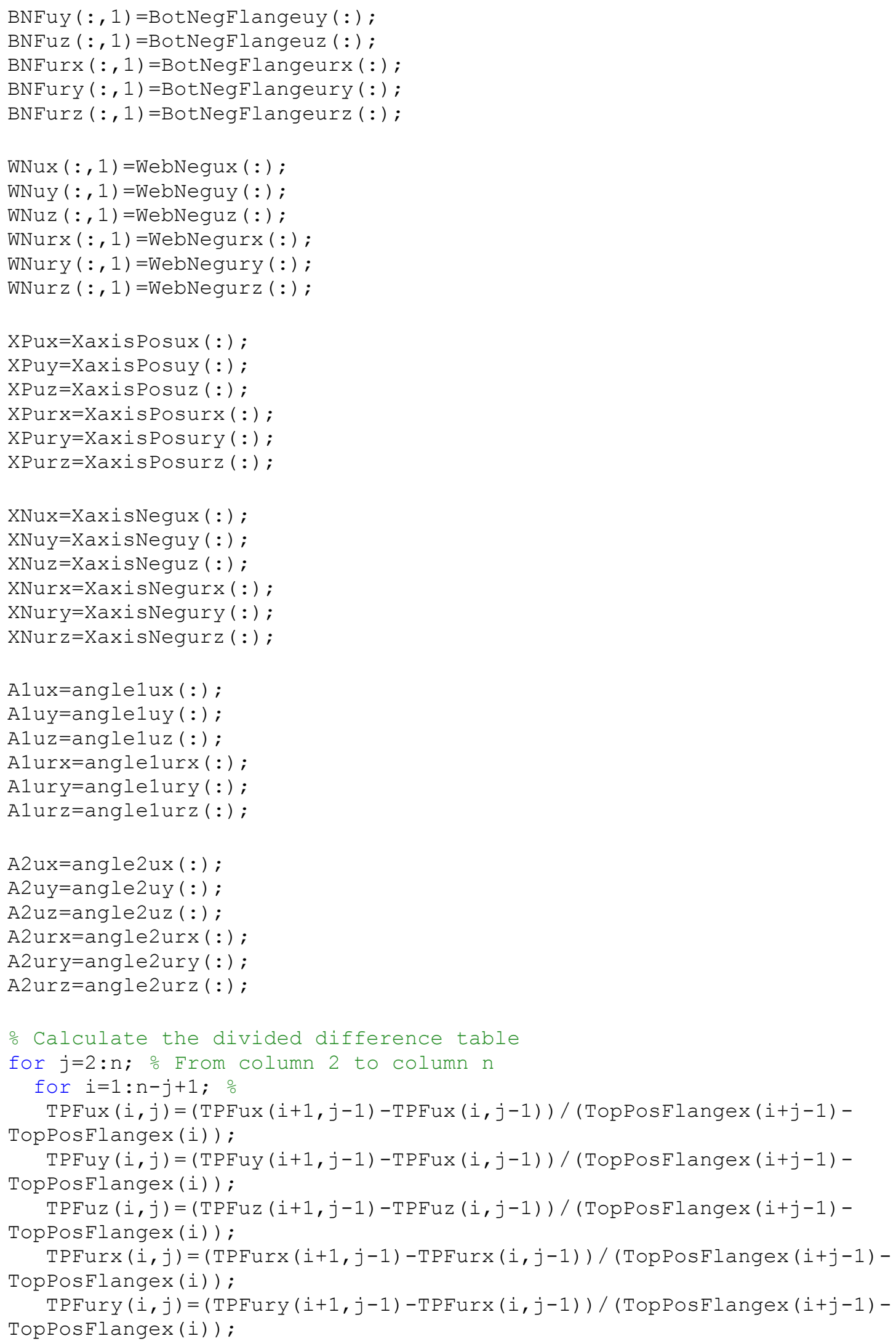


$\operatorname{TPFurz}(i, j)=(\operatorname{TPFurz}(i+1, j-1)-\operatorname{TPFurz}(i, j-1)) /(\operatorname{TopPosFlangex}(i+j-1)-$ TopPosflangex (i));

end

end

for $j=2: n 1 ; \%$ From column 2 to column $n$

for $i=1: n 1-j+1$; $\frac{\circ}{\circ}$

$\operatorname{BPFux}(i, j)=(\operatorname{BPFux}(i+1, j-1)-\operatorname{BPFux}(i, j-1)) /(\operatorname{BotPosFlangex}(i+j-1)-$

BotPosflangex(i)) ;

$\operatorname{BPFuy}(i, j)=(\operatorname{BPFuy}(i+1, j-1)-\operatorname{BPFux}(i, j-1)) /(\operatorname{BotPosFlangex}(i+j-1)-$

BotPosflangex (i));

$\operatorname{BPFuz}(i, j)=(\operatorname{BPFuz}(i+1, j-1)-\operatorname{BPFuz}(i, j-1)) /(\operatorname{BotPosFlangex}(i+j-1)-$ BotPosflangex (i));

$\operatorname{BPFurx}(i, j)=(\operatorname{BPFurx}(i+1, j-1)-\operatorname{BPFurx}(i, j-1)) /(\operatorname{BotPosFlangex}(i+j-1)-$ BotPosflangex (i)) ;

$\operatorname{BPFury}(i, j)=(\operatorname{BPFury}(i+1, j-1)-\operatorname{BPFurx}(i, j-1)) /(\operatorname{BotPosFlangex}(i+j-1)-$ BotPosflangex (i));

$\operatorname{BPFurz}(i, j)=(\operatorname{BPFurz}(i+1, j-1)-\operatorname{BPFurz}(i, j-1)) /(\operatorname{BotPosFlangex}(i+j-1)-$ BotPosflangex (i));

end

end

for $j=2: n 2 ; \%$ From column 2 to column $n$

for $i=1: n 2-j+1$;

$\operatorname{WPux}(i, j)=(\operatorname{WPux}(i+1, j-1)-\operatorname{WPux}(i, j-1)) /(\operatorname{WebPosy}(i+j-1)-\operatorname{WebPosy}(i))$;

$\operatorname{WPuy}(i, j)=(\operatorname{WPuy}(i+1, j-1)-\operatorname{WPux}(i, j-1)) /(\operatorname{WebPosy}(i+j-1)-\operatorname{WebPosy}(i)) ;$ $\operatorname{WPuz}(i, j)=(\operatorname{WPuz}(i+1, j-1)-\operatorname{WPuz}(i, j-1)) /(\operatorname{WebPosy}(i+j-1)-\operatorname{WebPosy}(i))$; $\operatorname{WPurx}(i, j)=(\operatorname{WPurx}(i+1, j-1)-\operatorname{WPurx}(i, j-1)) /(\operatorname{WebPosy}(i+j-1)-$

WebPosy(i));

$\operatorname{WPury}(i, j)=(\operatorname{WPury}(i+1, j-1)-\operatorname{WPurx}(i, j-1)) /(\operatorname{WebPosy}(i+j-1)-$

WebPosy (i)) ;

$\operatorname{WPurz}(i, j)=(\operatorname{WPurz}(i+1, j-1)-\operatorname{WPurz}(i, j-1)) /(\operatorname{WebPosy}(i+j-1)-$

WebPosy (i)) ;

end

end

for $j=2: n 3$; $\frac{\circ}{\circ}$ From column 2 to column $n$

for $i=1: n 3-j+1 ;$ \%

$\operatorname{TNFux}(i, j)=(\operatorname{TNFux}(i+1, j-1)-\operatorname{TNFux}(i, j-1)) /(\operatorname{TopNegFlangex}(i+j-1)-$ TopNegFlangex (i)) ;

$\operatorname{TNFuy}(i, j)=(\operatorname{TNFuy}(i+1, j-1)-\operatorname{TNFux}(i, j-1)) /(\operatorname{TopNegFlangex}(i+j-1)-$ TopNegFlangex (i)) ;

$\operatorname{TNFuz}(i, j)=(\operatorname{TNFuz}(i+1, j-1)-\operatorname{TNFuz}(i, j-1)) /(\operatorname{TopNegFlangex}(i+j-1)-$ TopNegFlangex (i)) ;

$\operatorname{TNFurx}(i, j)=(\operatorname{TNFurx}(i+1, j-1)-\operatorname{TNFurx}(i, j-1)) /(\operatorname{TopNegFlangex}(i+j-1)-$ TopNegFlangex (i));

$\operatorname{TNFury}(i, j)=(\operatorname{TNFury}(i+1, j-1)-\operatorname{TNFurx}(i, j-1)) /(\operatorname{TopNegFlangex}(i+j-1)-$ TopNegFlangex (i));

$\operatorname{TNFurz}(i, j)=(\operatorname{TNFurz}(i+1, j-1)-\operatorname{TNFurz}(i, j-1)) /(\operatorname{TopNegFlangex}(i+j-1)-$ TopNegFlangex (i)) ; end

end

for $j=2: n 4 ; \%$ From column 2 to column $n$

for $i=1: n 4-j+1 ;$ o

$\operatorname{BNFux}(i, j)=(\operatorname{BNFux}(i+1, j-1)-\operatorname{BNFux}(i, j-1)) /(\operatorname{BotNegFlangex}(i+j-1)-$ BotNegFlangex (i));

$\operatorname{BNFuy}(i, j)=(\operatorname{BNFuy}(i+1, j-1)-\operatorname{BNFux}(i, j-1)) /(\operatorname{BotNegFlangex}(i+j-1)-$ BotNegFlangex (i)); 
$\operatorname{BNFuz}(i, j)=(\operatorname{BNFuz}(i+1, j-1)-\operatorname{BNFuz}(i, j-1)) /(\operatorname{BotNegFlangex}(i+j-1)-$ BotNegFlangex (i));

$\operatorname{BNFurx}(i, j)=(\operatorname{BNFurx}(i+1, j-1)-\operatorname{BNFurx}(i, j-1)) /(\operatorname{BotNegFlangex}(i+j-1)-$ BotNegFlangex (i)) ;

$\operatorname{BNFury}(i, j)=(\operatorname{BNFury}(i+1, j-1)-\operatorname{BNFurx}(i, j-1)) /(\operatorname{BotNegFlangex}(i+j-1)-$ BotNegFlangex (i)) ;

$\operatorname{BNFurz}(i, j)=(\operatorname{BNFurz}(i+1, j-1)-\operatorname{BNFurz}(i, j-1)) /(\operatorname{BotNegFlangex}(i+j-1)-$ BotNegFlangex (i)) ;

end

end

for $j=2: n 5 ;$ From column 2 to column $n$

for $i=1: n 5-j+1 ; \%$

$\operatorname{WNux}(i, j)=(\operatorname{WNux}(i+1, j-1)-\operatorname{WNux}(i, j-1)) /(\operatorname{WebNegy}(i+j-1)-\operatorname{WebNegy}(i))$;

$\operatorname{WNuy}(i, j)=(\operatorname{WNuy}(i+1, j-1)-\operatorname{WNux}(i, j-1)) /(\operatorname{WebNegy}(i+j-1)-\operatorname{WebNegy}(i))$;

$\operatorname{WNuz}(i, j)=(\operatorname{WNuz}(i+1, j-1)-\operatorname{WNuz}(i, j-1)) /(\operatorname{WebNegy}(i+j-1)-\operatorname{WebNegy}(i))$;

$\operatorname{WNurx}(i, j)=(\operatorname{WNurx}(i+1, j-1)-\operatorname{WNurx}(i, j-1)) /(\operatorname{WebNegy}(i+j-1)-$

WebNegy (i));

$\operatorname{WNury}(i, j)=(\operatorname{WNury}(i+1, j-1)-\operatorname{WNurx}(i, j-1)) /(\operatorname{WebNegy}(i+j-1)-$

WebNegy $(i))$;

$\operatorname{WNurz}(i, j)=(\operatorname{WNurz}(i+1, j-1)-\operatorname{WNurz}(i, j-1)) /(\operatorname{WebNegy}(i+j-1)-$

WebNegy (i));

end

end

for $j=2: n 6$; $\frac{\circ}{\circ}$ From column 2 to column $n$

for $i=1: n 6-j+1 ; \%$

$\operatorname{XPux}(i, j)=(\operatorname{XPux}(i+1, j-1)-\operatorname{XPux}(i, j-1)) /(\operatorname{XaxisPosz}(i+j-1)-$

XaxisPosz (i));

$\operatorname{xPuy}(i, j)=(\operatorname{XPuy}(i+1, j-1)-\operatorname{XPux}(i, j-1)) /(\operatorname{XaxisPosz}(i+j-1)-$

XaxisPosz(i));

$\operatorname{XPuz}(i, j)=(\operatorname{XPuz}(i+1, j-1)-\operatorname{XPuz}(i, j-1)) /(\operatorname{XaxisPosz}(i+j-1)-$ XaxisPosz(i));

$\operatorname{XPurx}(i, j)=(\operatorname{XPurx}(i+1, j-1)-\operatorname{XPurx}(i, j-1)) /(\operatorname{XaxisPosz}(i+j-1)-$ XaxisPosz(i));

$\operatorname{xPury}(i, j)=(\operatorname{XPury}(i+1, j-1)-\operatorname{xPurx}(i, j-1)) /(\operatorname{XaxisPos} z(i+j-1)-$ XaxisPosz(i));

$\operatorname{XPurz}(i, j)=(\operatorname{XPurz}(i+1, j-1)-\operatorname{XPurz}(i, j-1)) /(\operatorname{XaxisPos} z(i+j-1)-$ XaxisPosz(i));

end

end

for $j=2: n 7$; From column 2 to column $n$

for $i=1: n 7-j+1 ;$ o

$\operatorname{XNux}(i, j)=(\operatorname{XNux}(i+1, j-1)-\operatorname{XNux}(i, j-1)) /(\operatorname{XaxisNegz}(i+j-1)-$

XaxisNegz (i));

$\operatorname{XNuy}(i, j)=(\operatorname{XNuy}(i+1, j-1)-\operatorname{XNux}(i, j-1)) /(\operatorname{XaxisNegz}(i+j-1)-$ XaxisNegz (i));

$\operatorname{XNuz}(i, j)=(\operatorname{XNuz}(i+1, j-1)-\operatorname{XNuz}(i, j-1)) /(\operatorname{XaxisNegz}(i+j-1)-$ XaxisNegz (i)) ;

$\operatorname{xNurx}(i, j)=(\operatorname{XNurx}(i+1, j-1)-\operatorname{XNurx}(i, j-1)) /(\operatorname{XaxisNegz}(i+j-1)-$ XaxisNegz (i));

$\operatorname{XNury}(i, j)=(\operatorname{XNury}(i+1, j-1)-\operatorname{XNurx}(i, j-1)) /(\operatorname{XaxisNegz}(i+j-1)-$ XaxisNegz (i));

$\operatorname{XNurz}(i, j)=(\operatorname{XNurz}(i+1, j-1)-\operatorname{XNurz}(i, j-1)) /(\operatorname{XaxisNegz}(i+j-1)-$ XaxisNegz (i));

end

end 


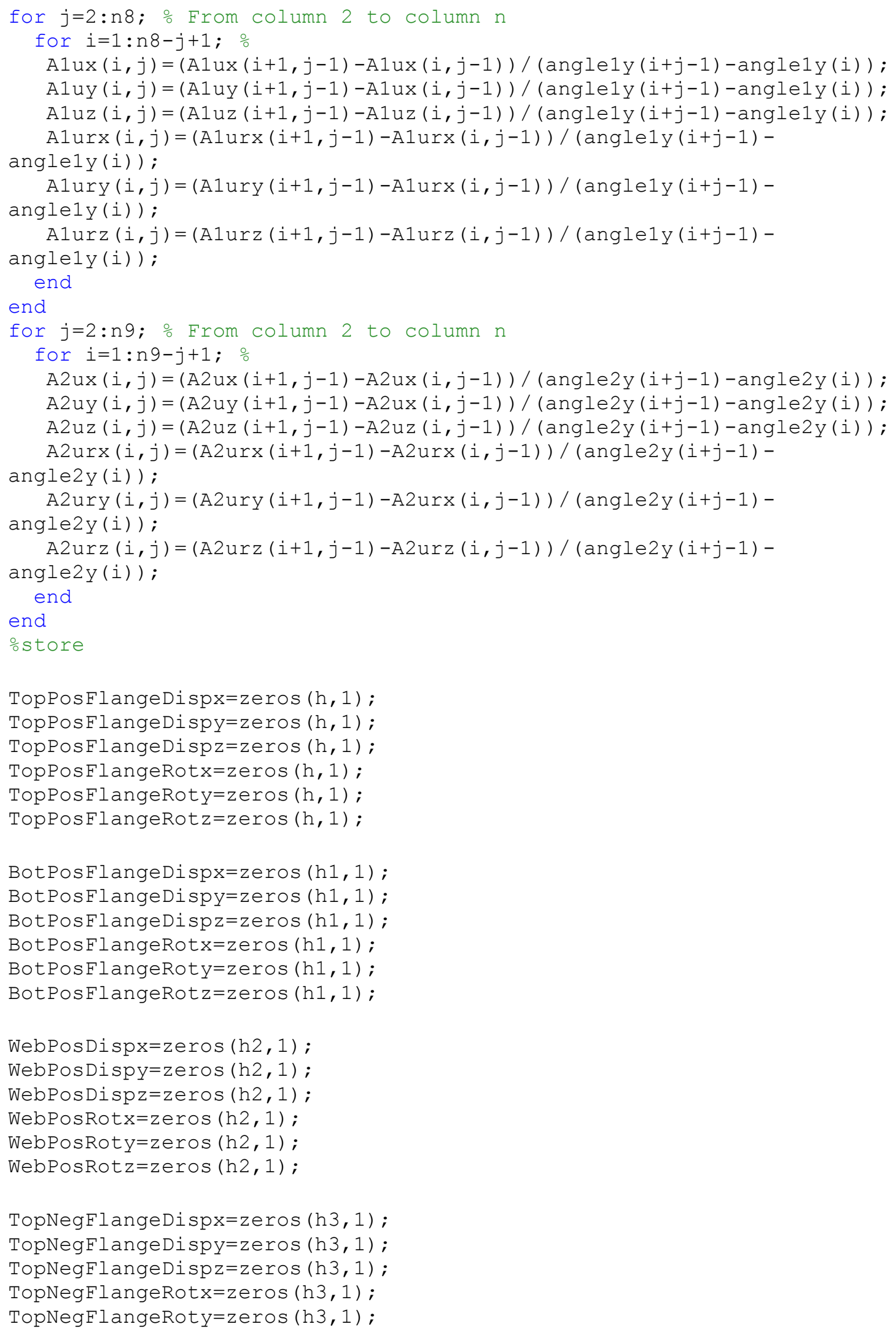




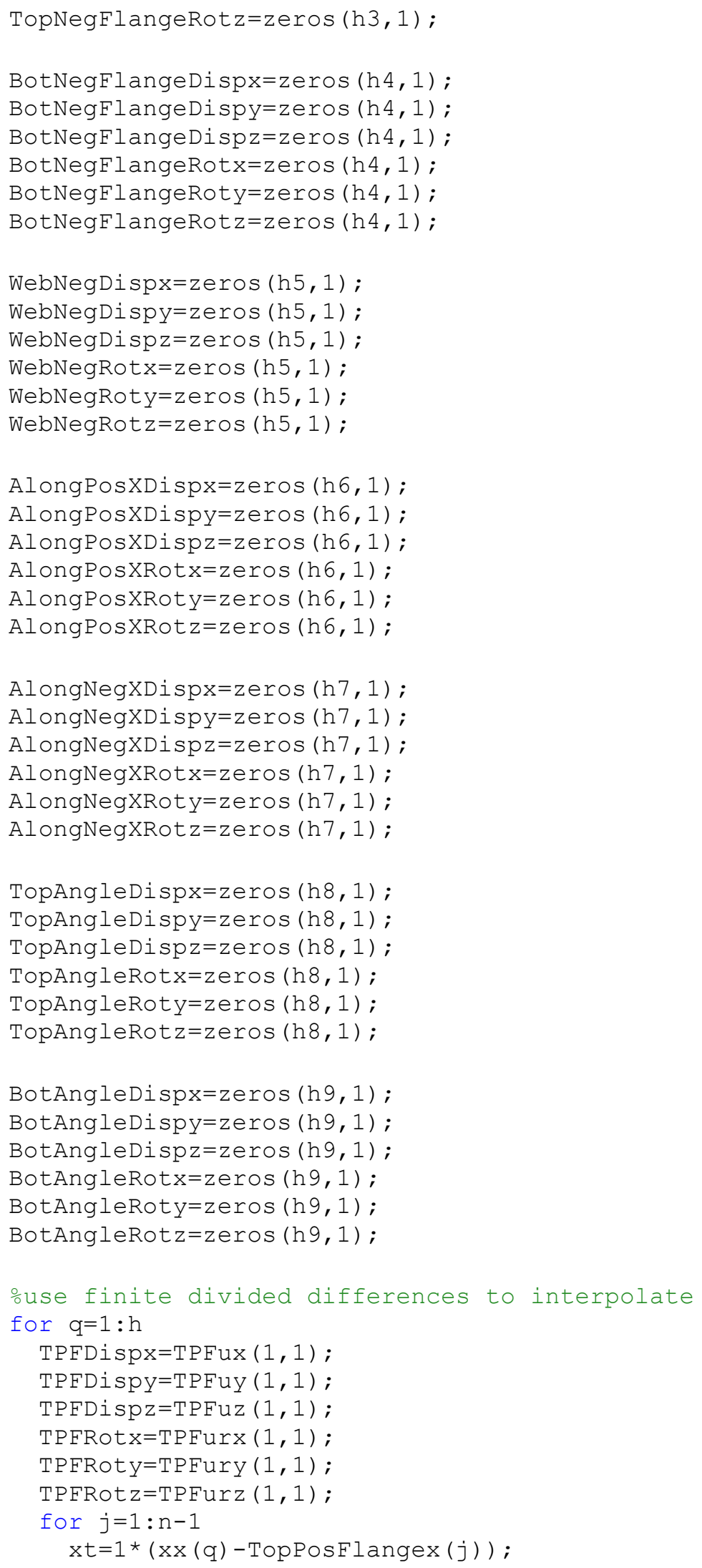




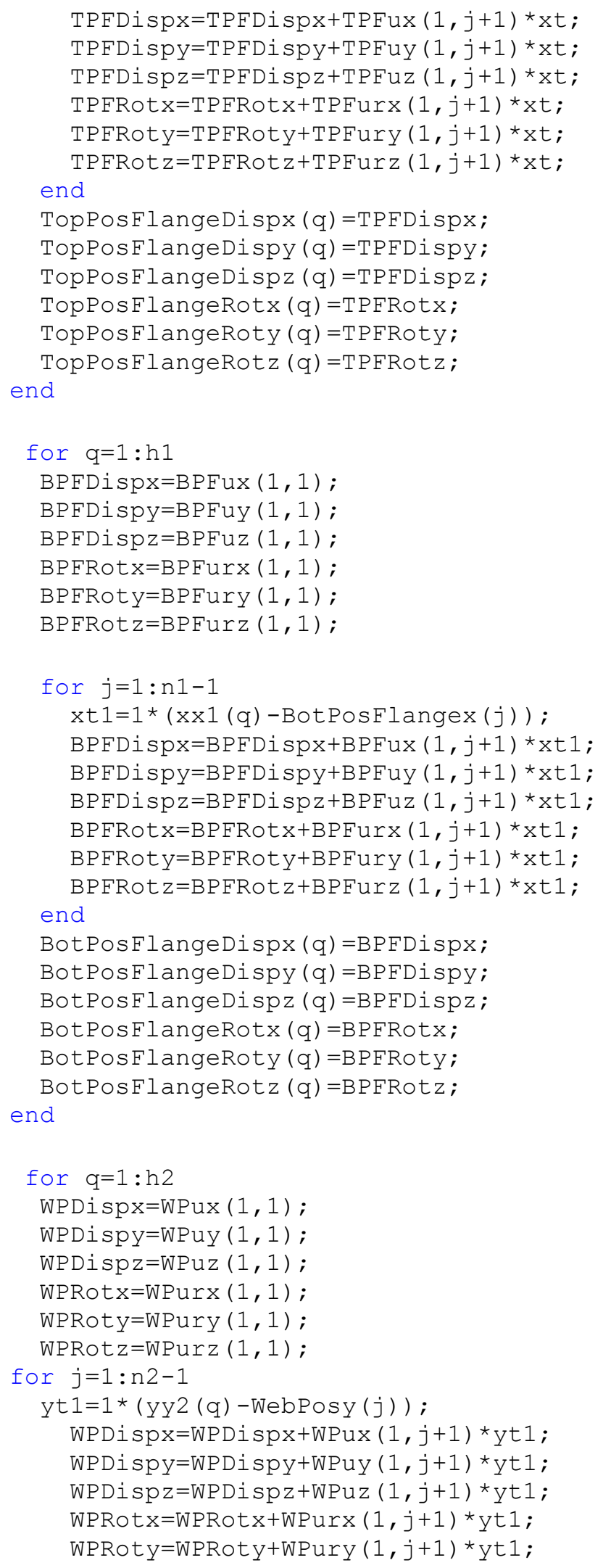




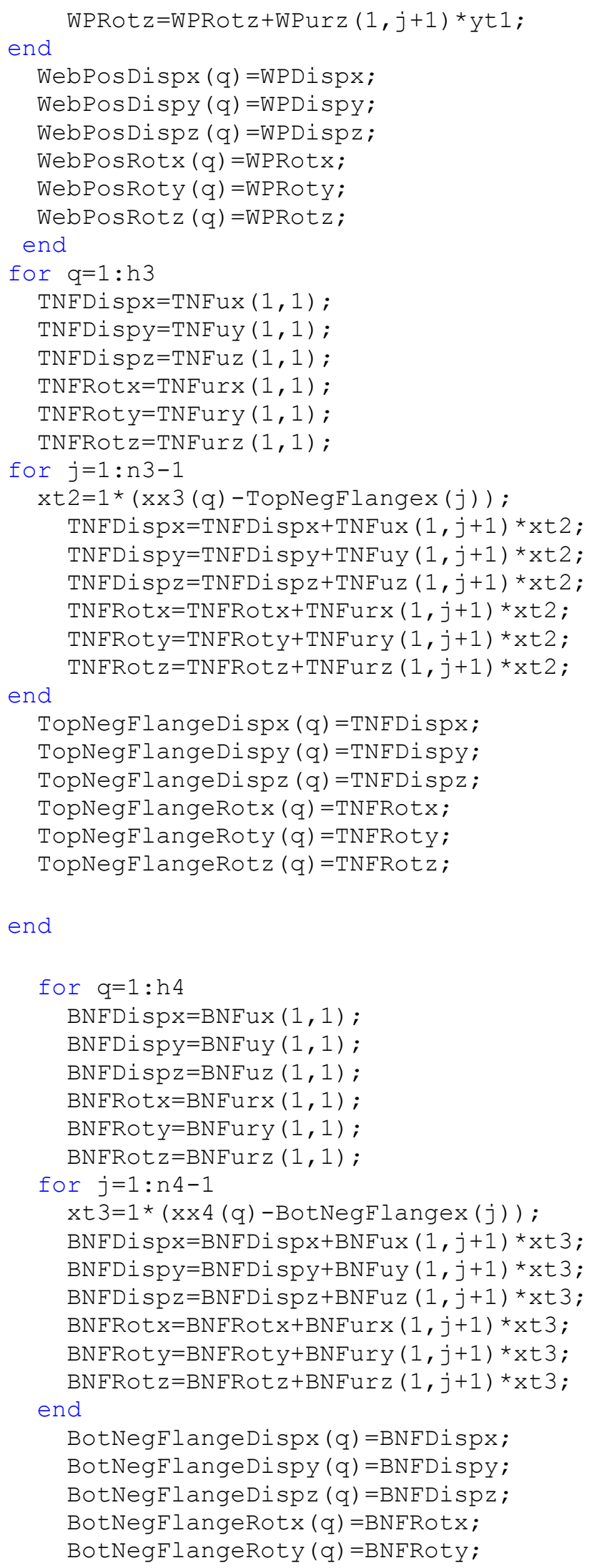




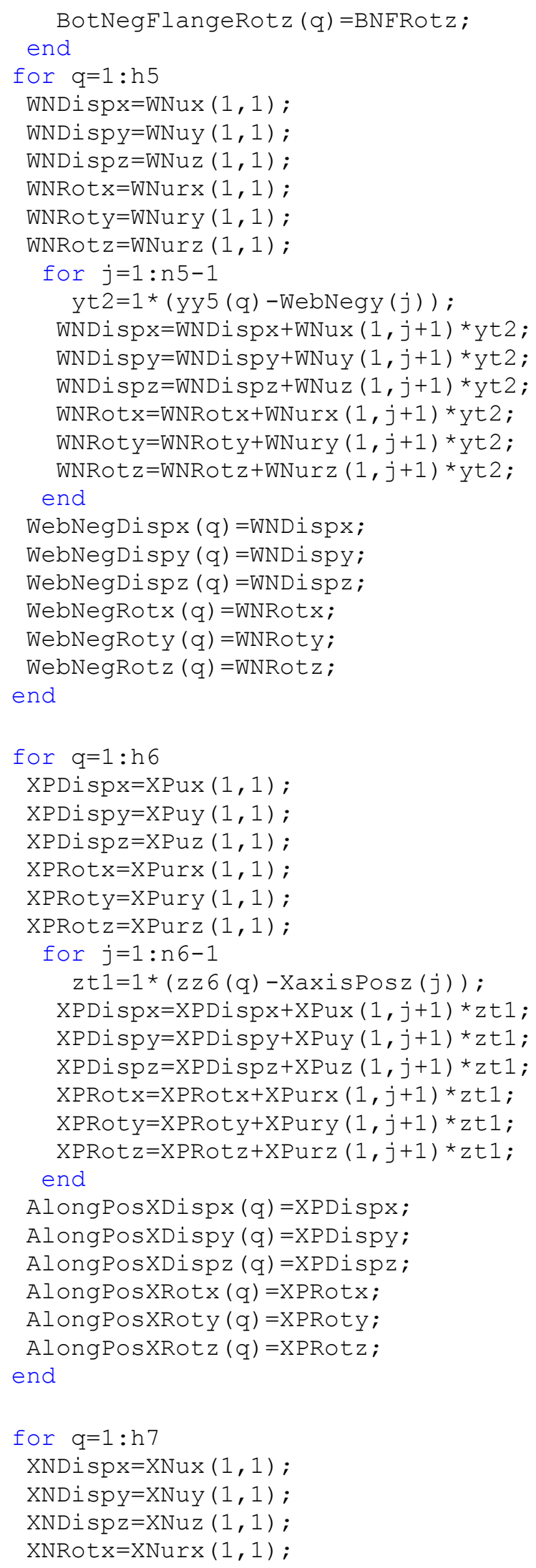




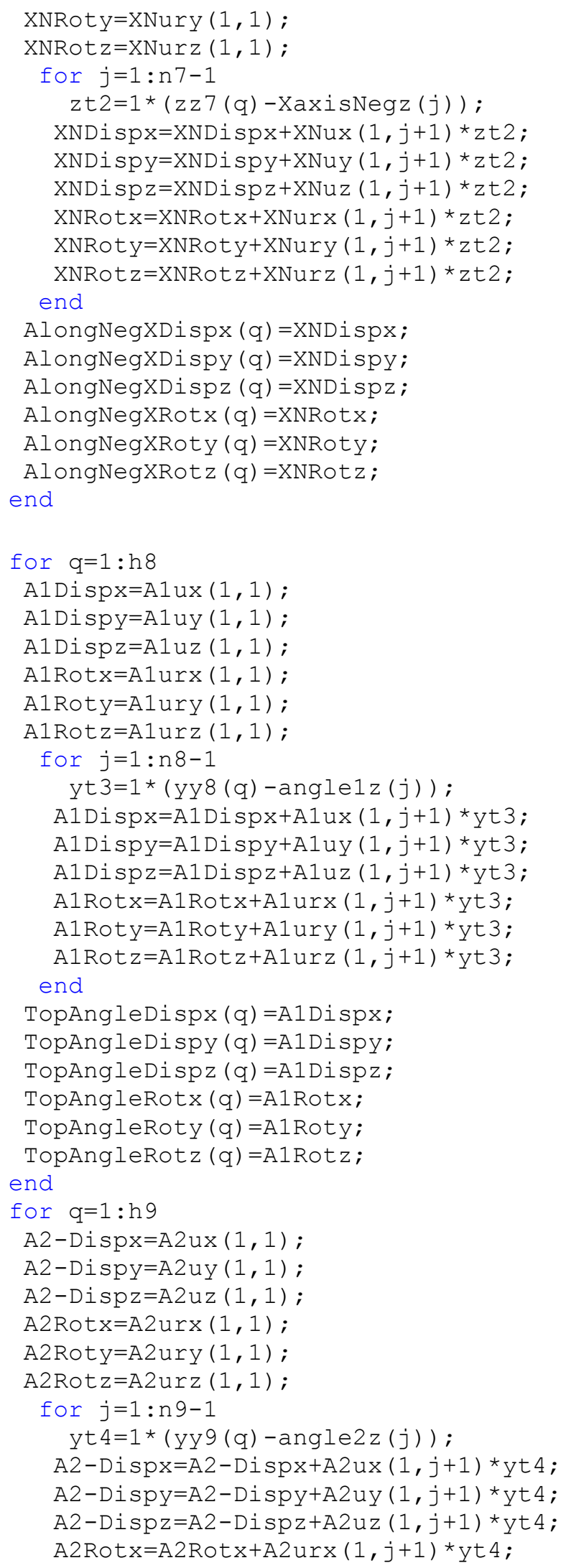









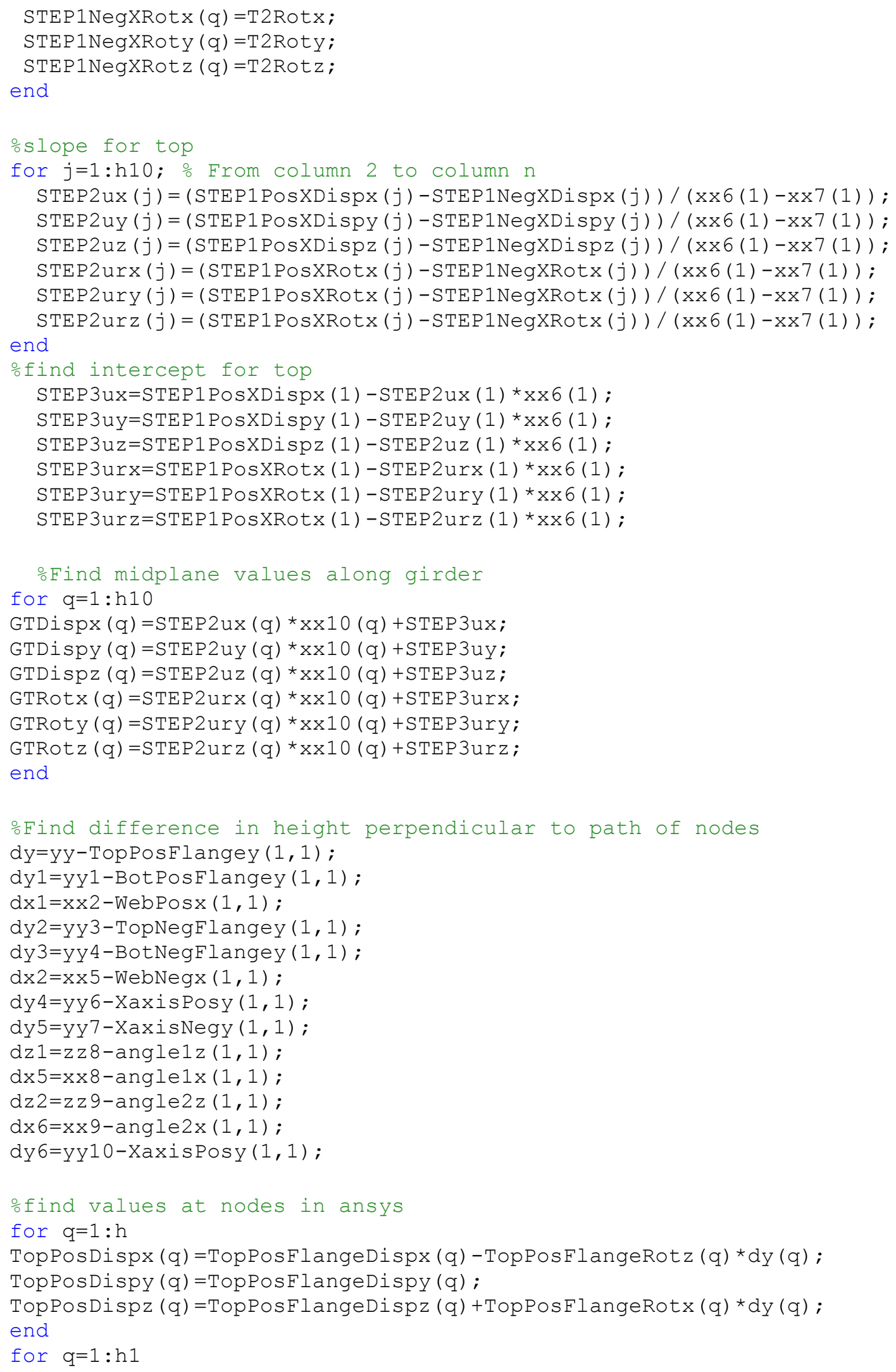




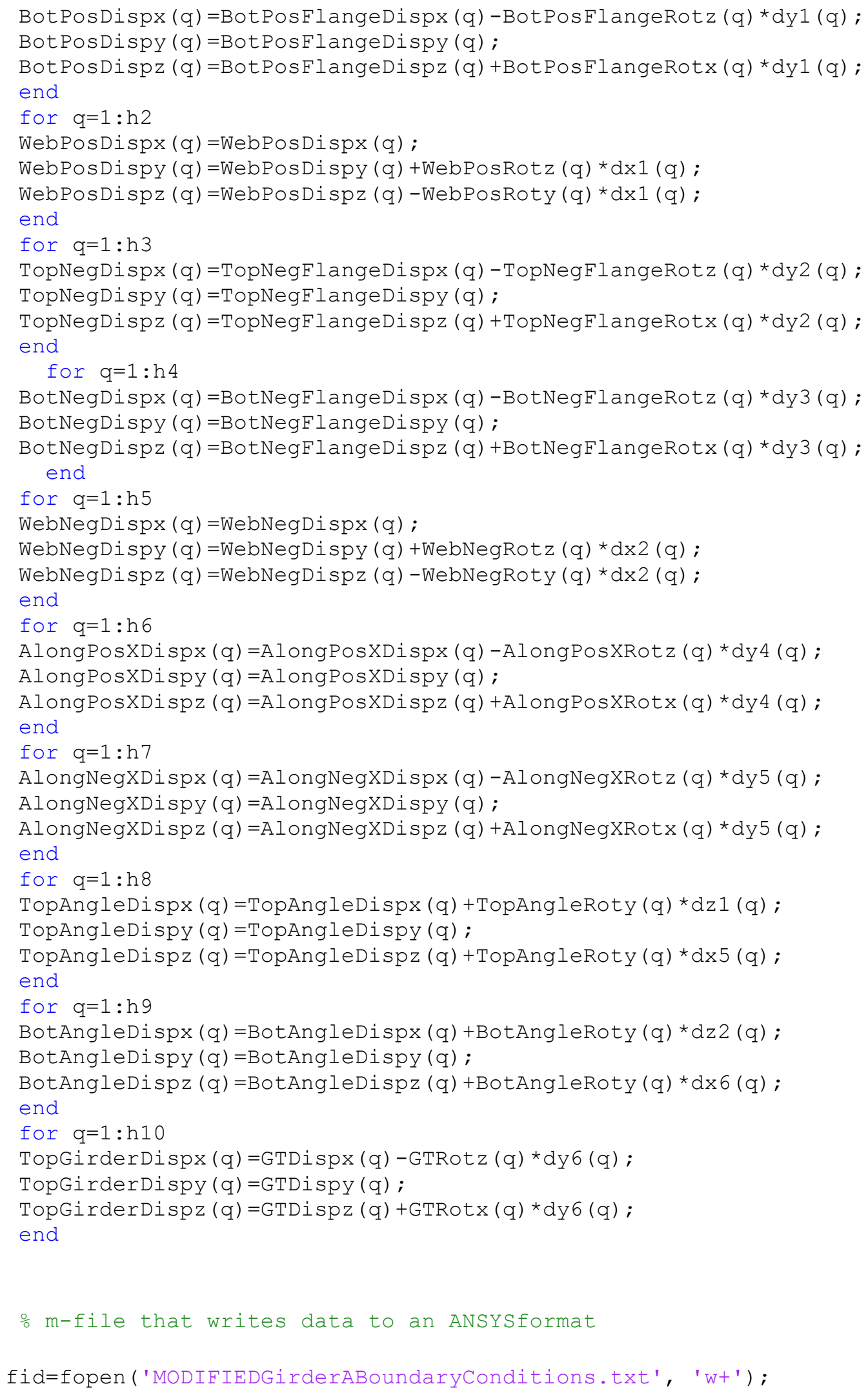




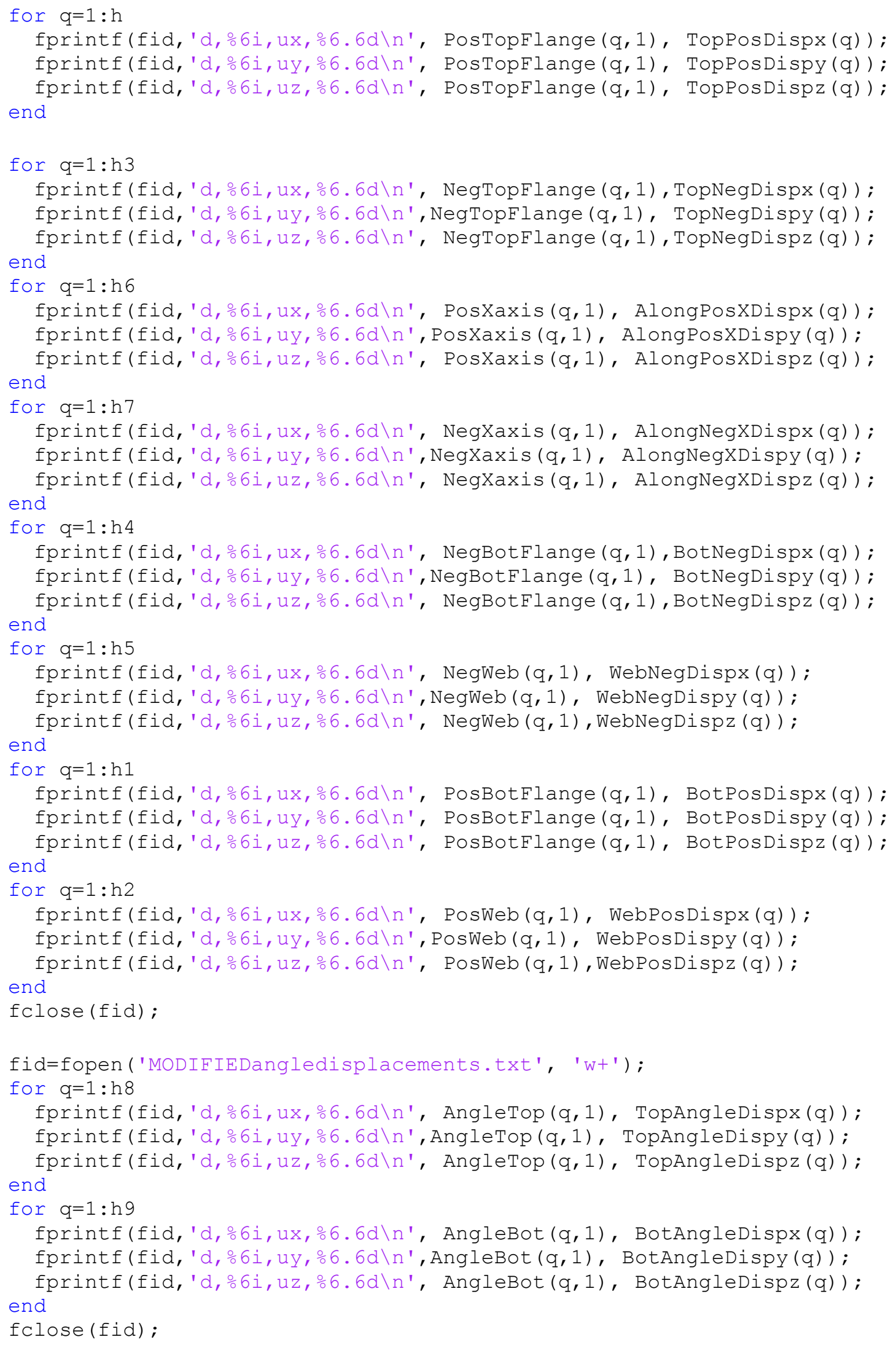




\subsection{The Carter Glass Bridge Plans and Photos}

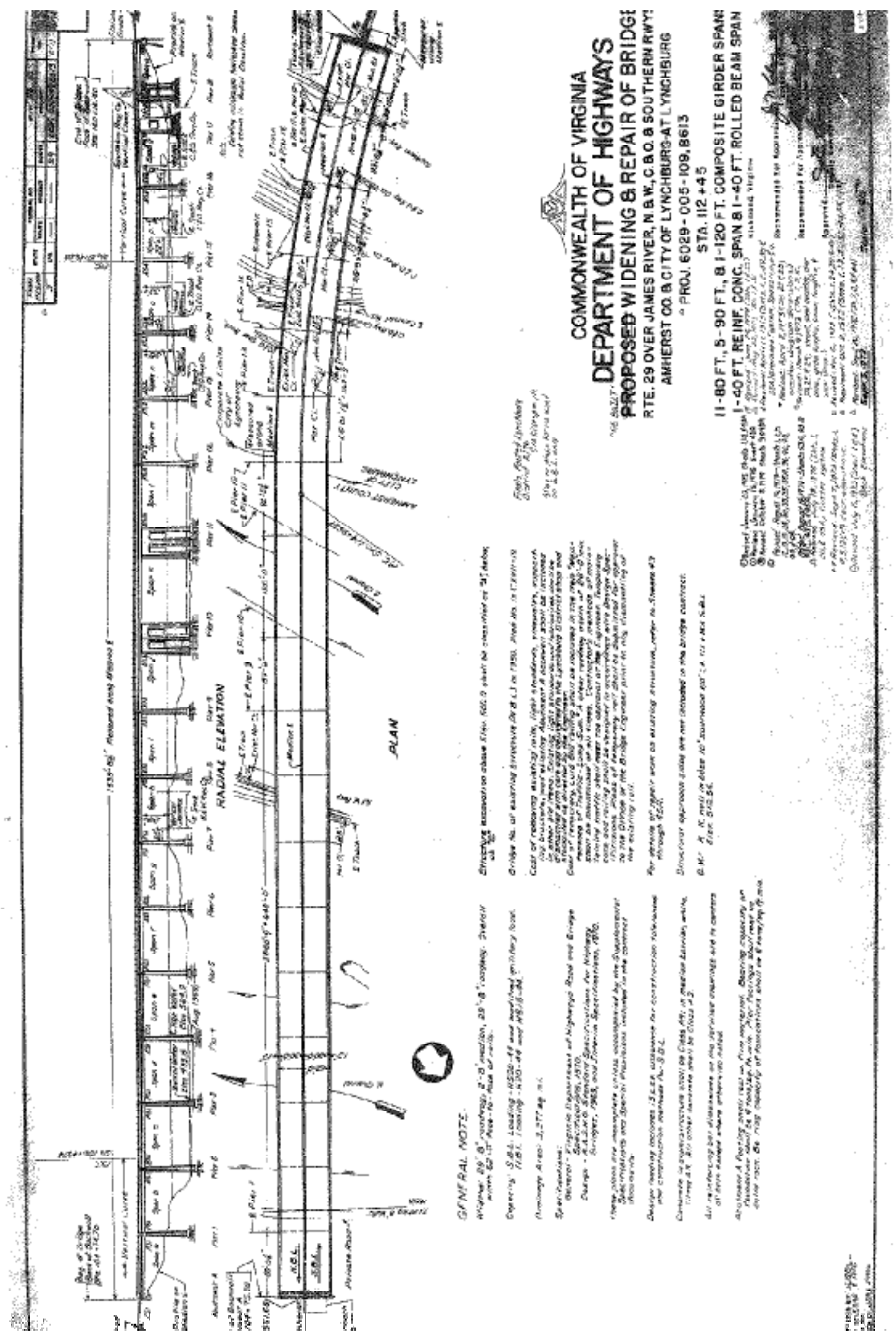




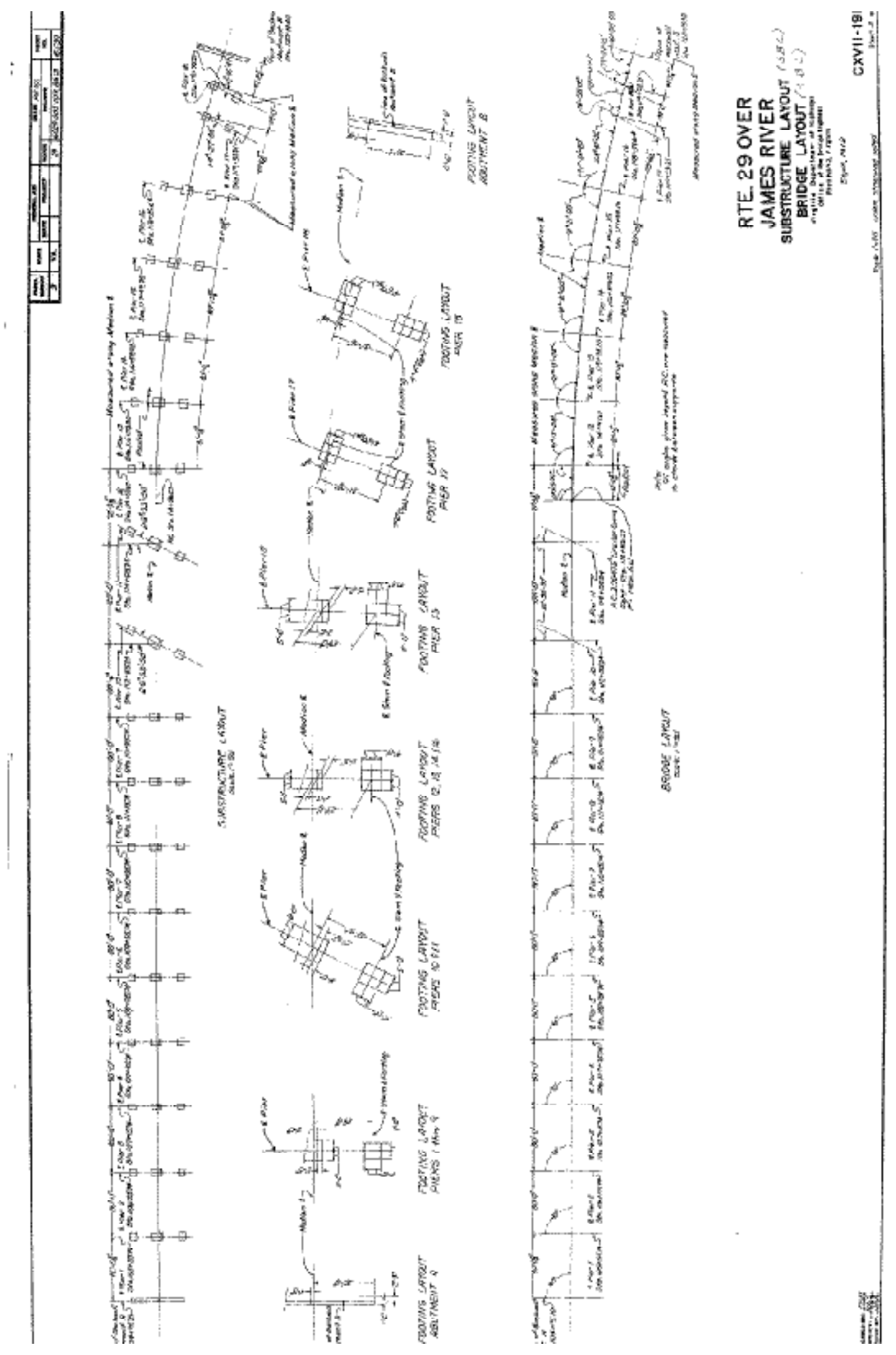




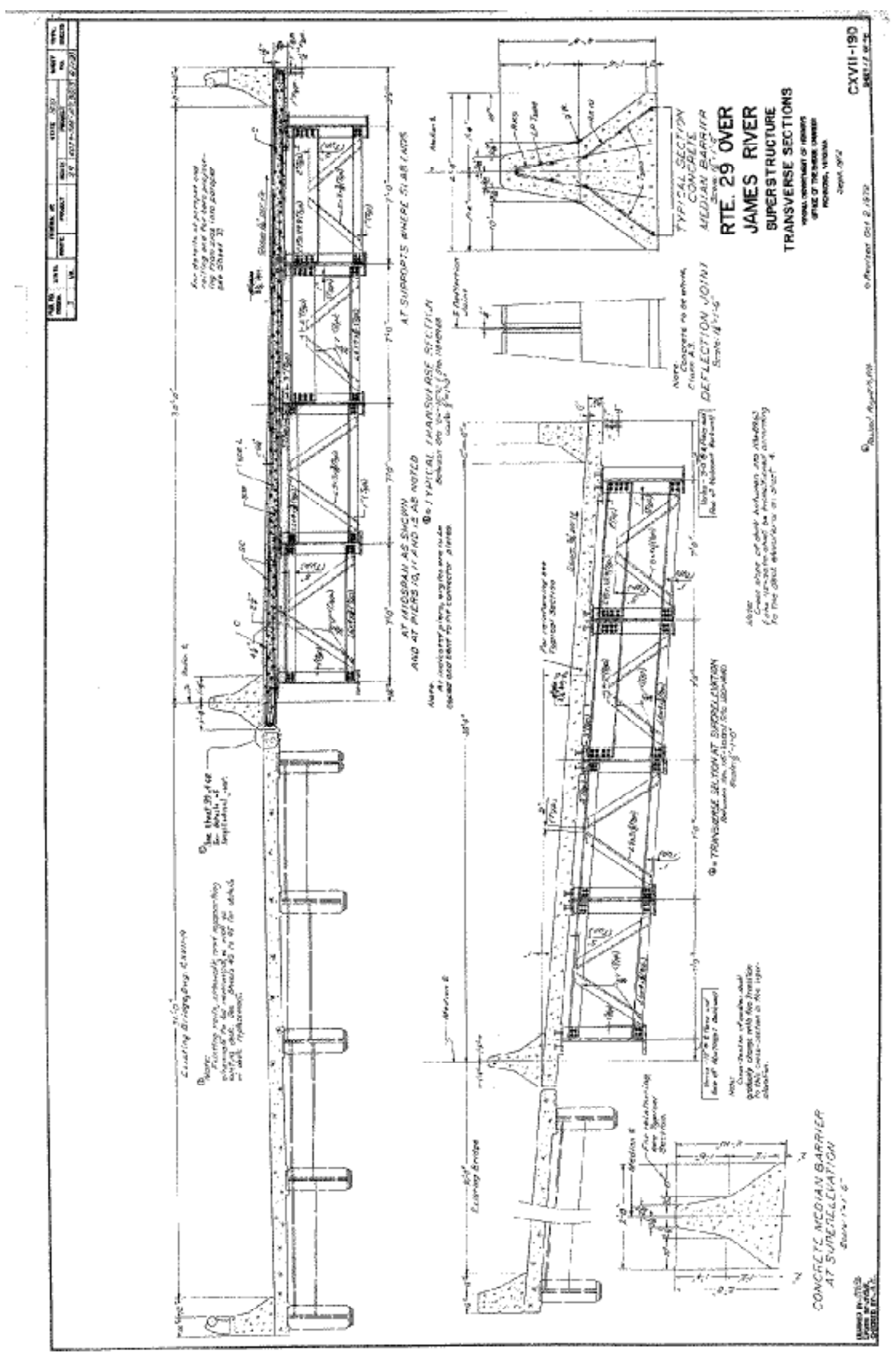




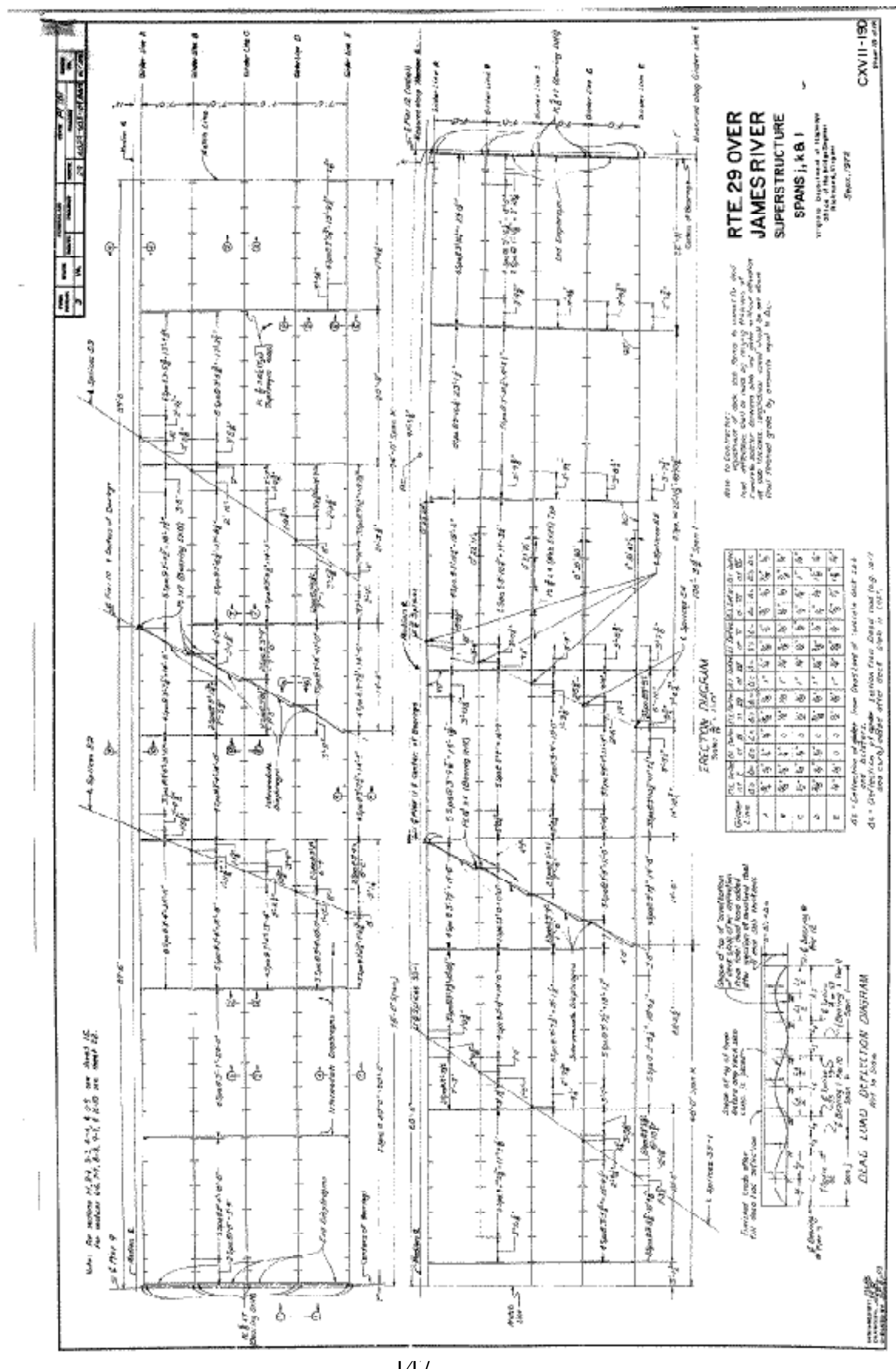

$14 /$ 


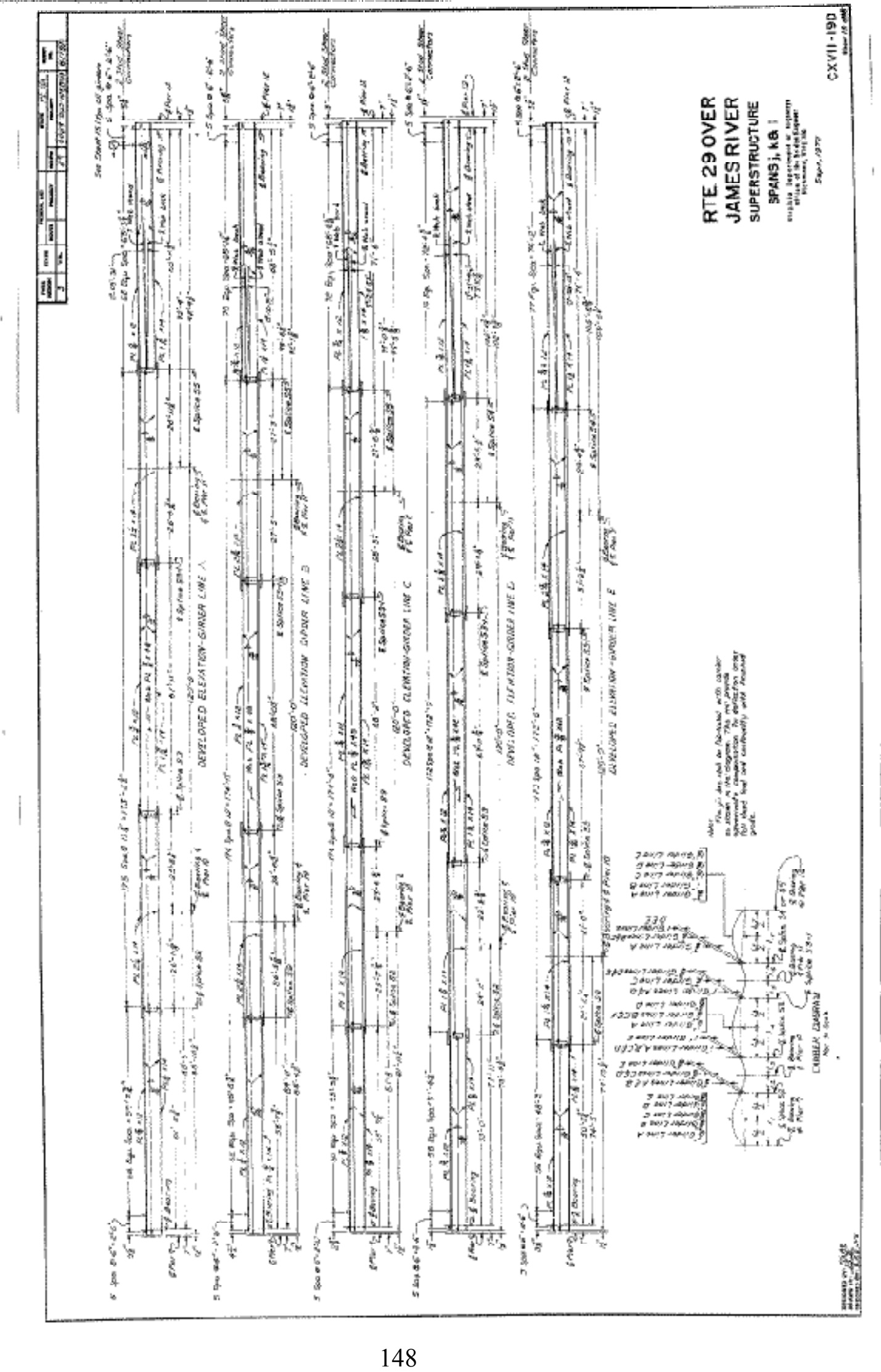




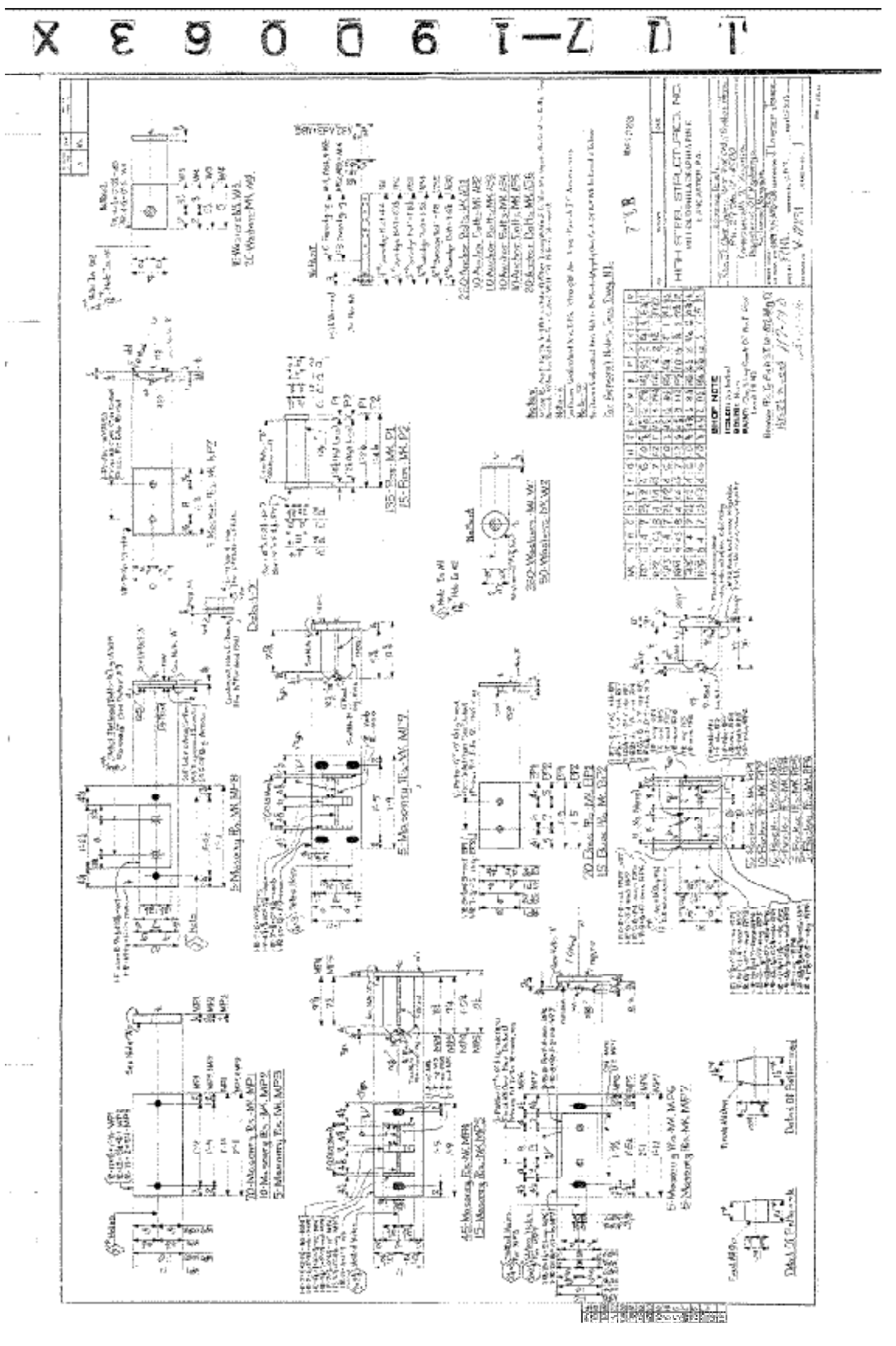



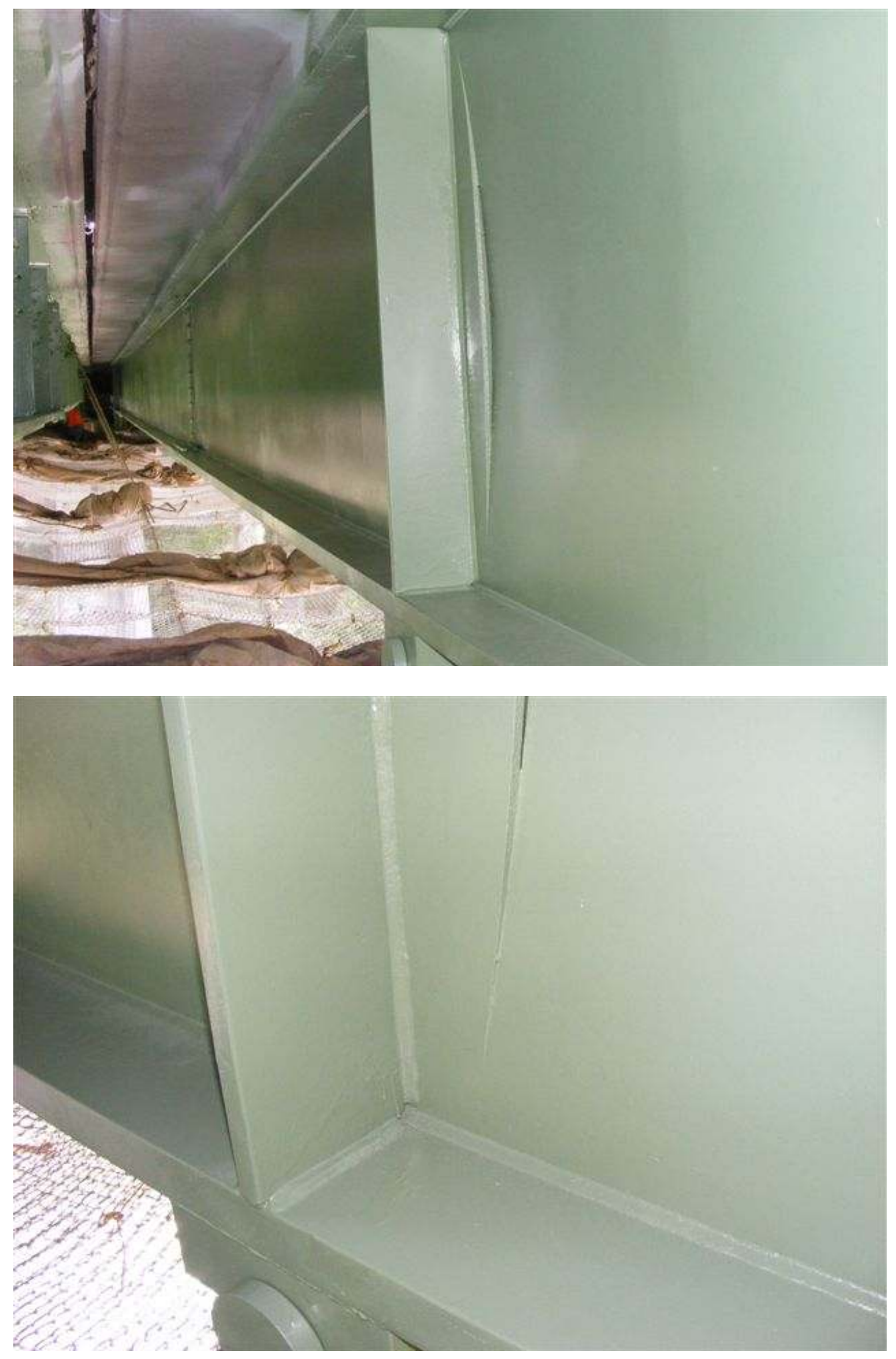